\title{
Aligned Continuous Cylindrical Pores Derived from Electrospun Polymer Fibers in Titanium Diboride
}

\author{
David Cyprian Hicks
}

\begin{abstract}
Thesis submitted to the faculty of the Virginia Polytechnic Institute and State University in
\end{abstract} partial fulfillment of the requirements for the degree of

\author{
Master of Science \\ in \\ Materials Science and Engineering
}

Carolina Tallon, Chair

Guoliang Liu

Gary D. Seidel

December 11, 2018

Blacksburg, Virginia

Keywords: Ultra High Temperature Ceramic, UHTC, Titanium Diboride, $\mathrm{TiB}_{2}$, Processing, Thermal Conductivity, Mechanical Testing, Multi-scale porous 


\title{
Aligned Continuous Cylindrical Pores Derived from Electrospun Polymer Fibers in Titanium
}

\section{Diboride}

\author{
David Cyprian Hicks
}

\begin{abstract}
The use of electrospun polystyrene (PS) fibers to create continuous long range ordered multiscale porous structures in titanium diboride $\left(\mathrm{TiB}_{2}\right)$ is investigated in this work. The introduction of electrospun PS fibers as a sacrificial filler into a colloidal suspension of $\mathrm{TiB}_{2}$ allows for easy control over the pore size, porosity, and long range ordering of the porous structures of the sintered ceramic. Green bodies were formed by vacuum infiltrating an electrospun-fiber-filled mold with the colloidal $\mathrm{TiB}_{2}$ suspension. The size, volume, distribution, and dispersion of the pores were optimized by carefully selecting the sacrificial polymer, the fiber diameter, the solvent, and the solid content of $\mathrm{TiB}_{2}$. The green bodies were partially sintered at $2000{ }^{\circ} \mathrm{C}$ in argon to form a multiscale porous structure via the removal of the PS fibers. Aligned continuous cylindrical pores were derived from the PS fibers in a range of $\sim 5-20 \mu \mathrm{m}$ and random porosity was revealed between the ceramic particles with the size of $\sim 0.3-1 \mu \mathrm{m}$. $\mathrm{TiB}_{2}$ near-net-shaped parts with the multi-scale porosities ( $\sim 0$ to $70 \%$ ) were successfully cast and sintered. The multi-scale porous structure produced from electrospun fibers was characterized both thermally and mechanically, at room temperature. The conductivity ranged from 12-31 $W \mathrm{~m}^{-1} \mathrm{~K}^{-1}$ at room temperature and the compressive strength ranged from 2-30 $\mathrm{MPa}$ at room temperature. Analytical thermal and mechanical models were employed to understand and verify he processing-structure-properties relationship. Finally, a method was devised for estimating the effective thermal conductivity of candidate materials for UHTC applications at relevant temperatures using a finite difference model and a controlled sample environment. This low-cost processing technique facilitates the production of thermally and mechanically anisotropic structures into near-net shape parts, for extreme environment applications, such as ultra high temperature insulation and active cooling components.
\end{abstract}




\title{
Aligned Continuous Cylindrical Pores Derived from Electrospun Polymer Fibers in Titanium Diboride
}

\author{
David Cyprian Hicks
}

\section{GENERAL AUDIENCE ABSTRACT}

Society is on the cusp of hypersonic flight which will revolutionize defense, space and transport technologies. Hypersonic flight is associated with conditions like that of atmospheric re-entry, high heat and force or specific locations of a space craft. The realization of hypersonic flight relies on innovative materials to survive the harsh conditions for repeated flight. We have created a new material with tiny holes that can help prevent heat flow from the harsh atmosphere from damaging the hypersonic craft. Thesis tiny holes are made from placing a polymer fiber in an advanced ceramic (which withstand high temperatures) and removing the fiber to leave holes. The tiny hole's effect on strength and heat flow have been studied, to understand how the tiny holes can be made better. It is difficult to test materials in the harsh atmosphere associated with hypersonic flight, so a program has been written to estimate thermal properties of candidate materials for hypersonic flight. 


\section{Acknowledgements}

I would like to acknowledge all those who contributed tangibly and intangibly. Thanks to my family and friends who were accommodating and supportive during this period. Their support was much appreciated and had great impact.

Many research groups and individuals contributed to this project. Thanks to Dr. Liu for his support and mentorship since I was an ungraduated research assistant in his lab. Thanks to all those in the Liu group that I had the privilege to work with: Assad Kahn for allowing me to work with him and providing academic guidance, Dr. Zhou for his creative and technical expertise in making the electrospinning aspect of this project possible, Joel and Tianyu for their help and support with electrospining. I would like to thank several within my larger group of Thanks to all those in the Advanced Manufacturing Team, and specifically: Jake Fallon and Cailean Pritchard for their help 3D printing necessary parts, Eric Gilmer for his help with rheological measurements. My research group Tallon Lab was instrumental in the completion of this project, specifically Chris Garza for helping with sintering and distributing many of the lab responsibilities and Kevin Gjata for helping with rheological measurements.

Thank you to all those in the Virginia Tech Materials Science and Engineering department for their help and encouragement through all of this. The advising of Kim Grandstaff has been superb and essential in meeting all the deadlines and finding resources for everything graduate school can throw at you. Dr. Staley, Dr. Corcoran, Dr. Suchicital and Dr. Reynolds were indispensable in elucidating materials science concepts and aiding with instrument operation and interpretation. Thanks to Zahra Gallagher from the Advanced Materials Group for her help with polymer characterization early in the project.

I would like to thank external agencies that contributed to the success of this project. Thanks to Dr. Wang from Oak Ridge National Lab for his essential aid with the high temperature thermal measurements, and comprehension of the data. The ICTAS nano characterization and fabrication lab (NCFL) was paramount in their characterization ability, education and guidance with materials problems, specifically Dr. McCartney for his expertise and conversation. Thanks to Teresa Porri (the computed tomography facility manager at the Cornell Biotechnology Resource Center Imaging Facility, Cornell University) for her help in creating 3D reconstructions of the porous structure in this work.

I would like to acknowledge the awards and associates that showed support: The American Chemical Scholar's Program in association with the Merck Scholar's Program, the Paul E. 
Torgersen Excellence in Graduate Research Award, and the Dr. Gary S. Clevinger, Sr. Memorial Endowed Scholarship for the financial support for his M.S. Thesis. Thank you to the International Conference and Exposition on Advanced Ceramics and Composites (ICACC 2018) for giving me the opportunity to present my work, as well as a travel grant. Thank you to International Journal of Applied Ceramic Technology for publishing chapter 1 of this thesis.

Lastly I would like to thank Dr. Tallon my advisor for her mentorship and advice throughout the past two and a half years. I have learned many invaluable lessons from her. I hope I can be as good of a mentor and example in the future as she has been to me. I have been helped by a multitude of individuals and organizations throughout this process. Isaac Newton said: "If I have seen further it is by standing on the shoulders of Giants" in my case those giants were the foundation of discoveries before me as well as the support of many. 


\section{Contents}

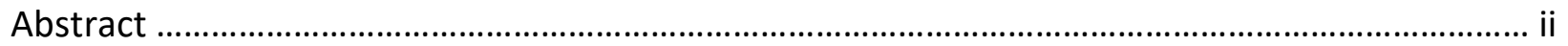

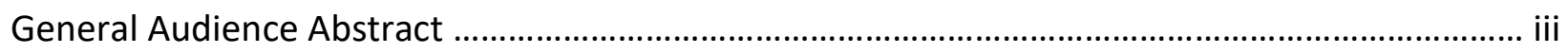

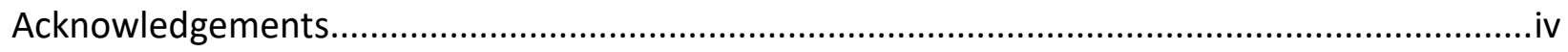

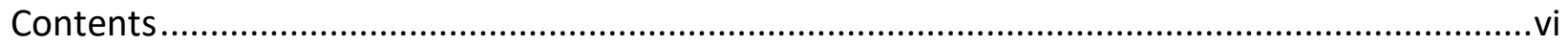

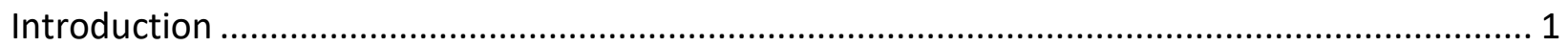

0.1.1 Hypersonic Environment and UHTC Properties............................................ 1

0.1 .2 UHTCs Materials for Hypersonic Environments ......................................... 2

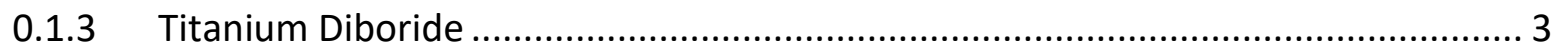

0.1.4 Challenges Facing the Application of UHTCs in Hypersonic Environments............. 4

0.1.5 Thermal Conductivity and Influence on UHTC Performance............................. 6

0.1.6 Porous UHTC Properties and Characterization ................................................ 8

0.1.7 UHTC Testing in Relevant Environments ................................................ 9

0.1.8 Processing Routes to Anisotropic Porous Structures ...................................... 10

0.1.9 Electrospinning as a New Sacrificial Template ........................................... 13

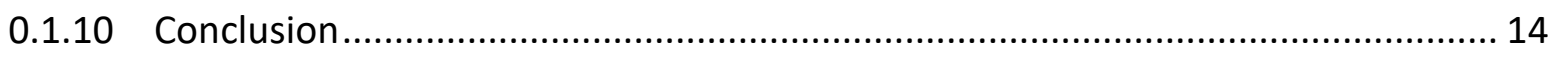

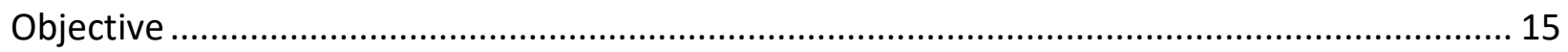

Chapter 1: Aligned Continuous Cylindrical Pores Derived from Electrospun Polymer Fibers in

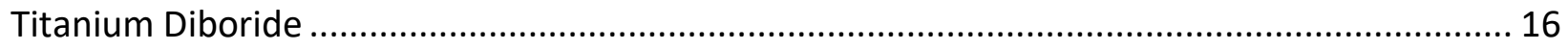

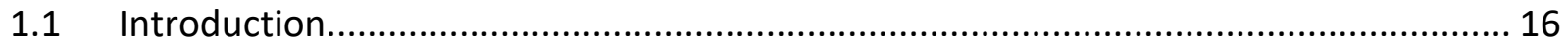

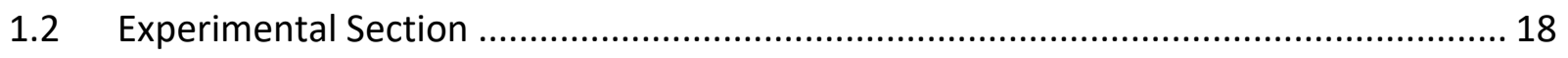

1.2.1 Ceramic Suspension Preparation ........................................................... 18

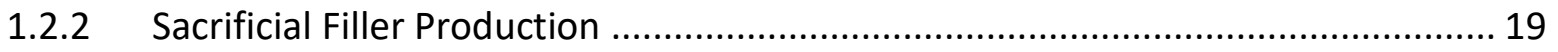

1.2.3 Vacuum Infiltration Casting (VIC) Rig...................................................... 21 


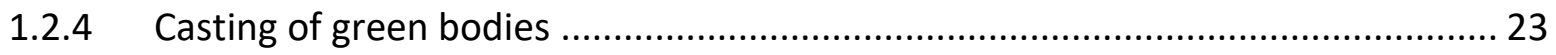

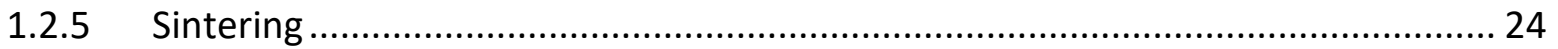

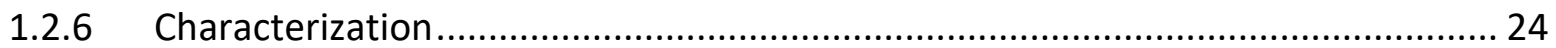

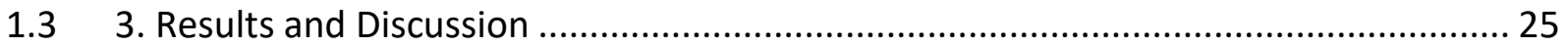

1.3.1 Effect of Size and Amount of the Electrospun Sacrificial Filler on Porosity ........... 25

1.3.2 Advantages of Vacuum Infiltration Casting with Electrospun Fibers as Sacrificial Fillers 33

1.4 Conclusions

Chapter 2: Mechanical and Thermal Characterization of Continuous Aligned Porous TiB $2 \ldots \ldots \ldots . . .39$

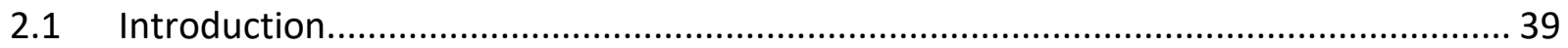

2.1.1 Heat Transfer in Porous Solids ......................................................................... 40

2.1.2 Thermal Conductivity of Parallel Cylindrical Pores ................................................ 44

2.1.3 Interparticle Porosity Thermal Conductivity ......................................................... 45

2.1.4 Mechanical Strength in Porous Solids .............................................................. 46

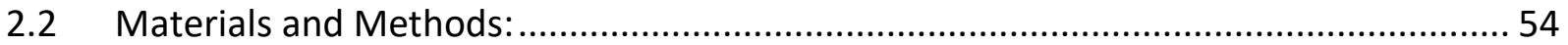

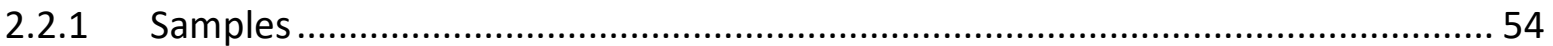

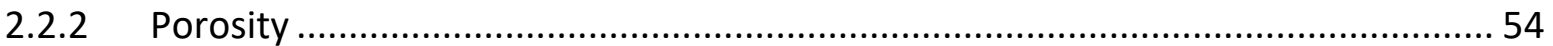

2.2.3 X-ray Computed Tomography (X-ray CT) ……................................................ 55

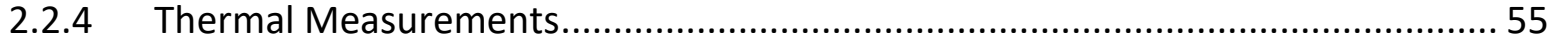

2.2.5 Mechanical Testing ......................................................................................... 56

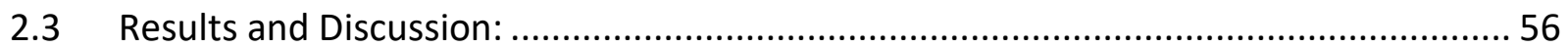

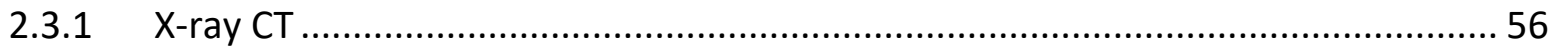

2.3.2 Thermal Conductivity ......................................................................................... 59

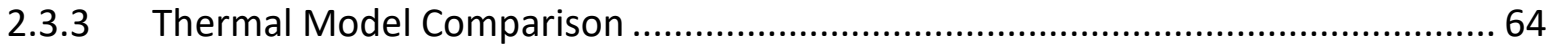

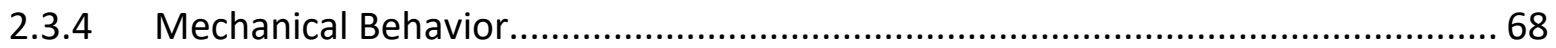


2.3.5 Mechanical Model for Comparison ............................................................ 75

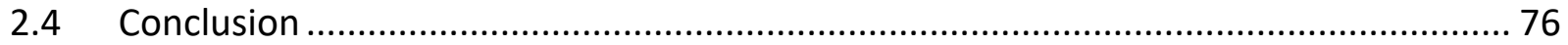

Chapter 3: A Model for Thermal Characterization of UHTC Materials at Simulated Hypersonic Conditions 77

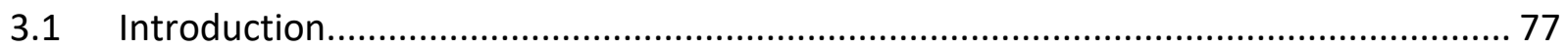

3.1.1 Thermal Model Under Relevant Conditions ............................................. 77

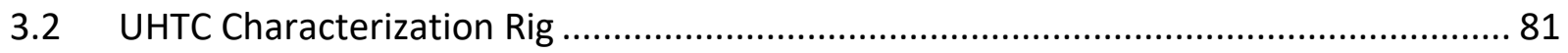

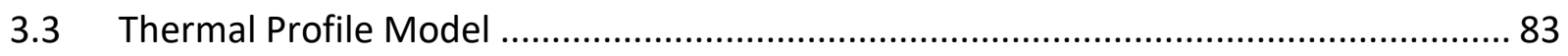

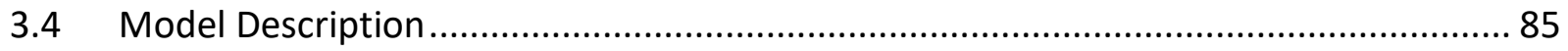

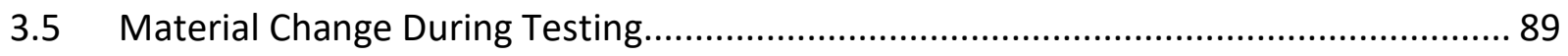

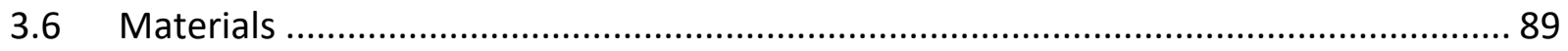

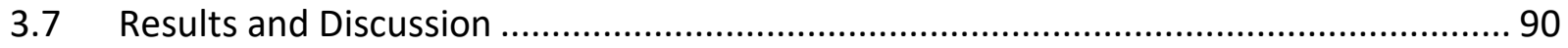

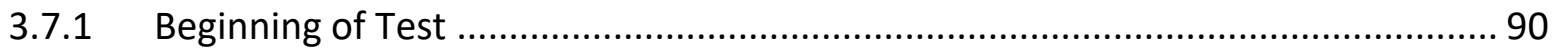

3.7.2 Equilibrium Temperature Profiles.............................................................. 94

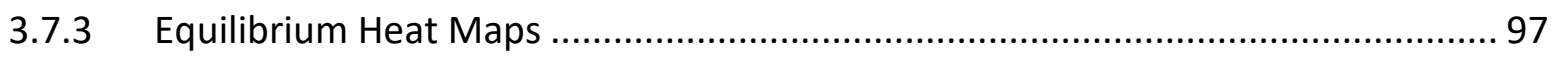

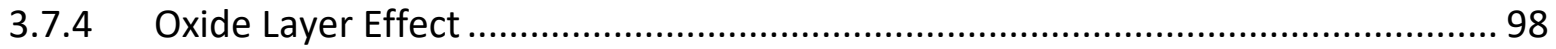

3.7.5 Effective Thermal Conductivity .............................................................. 100

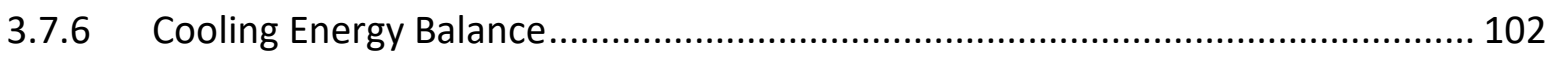

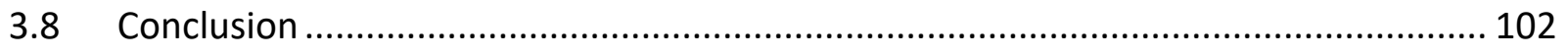

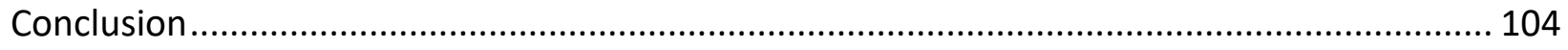

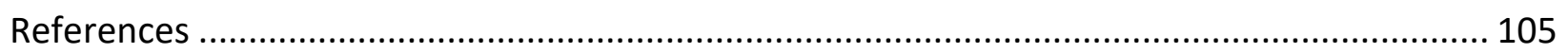

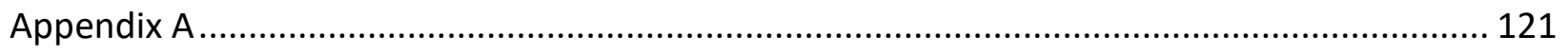




\section{Introduction}

\subsubsection{Hypersonic Environment and UHTC Properties}

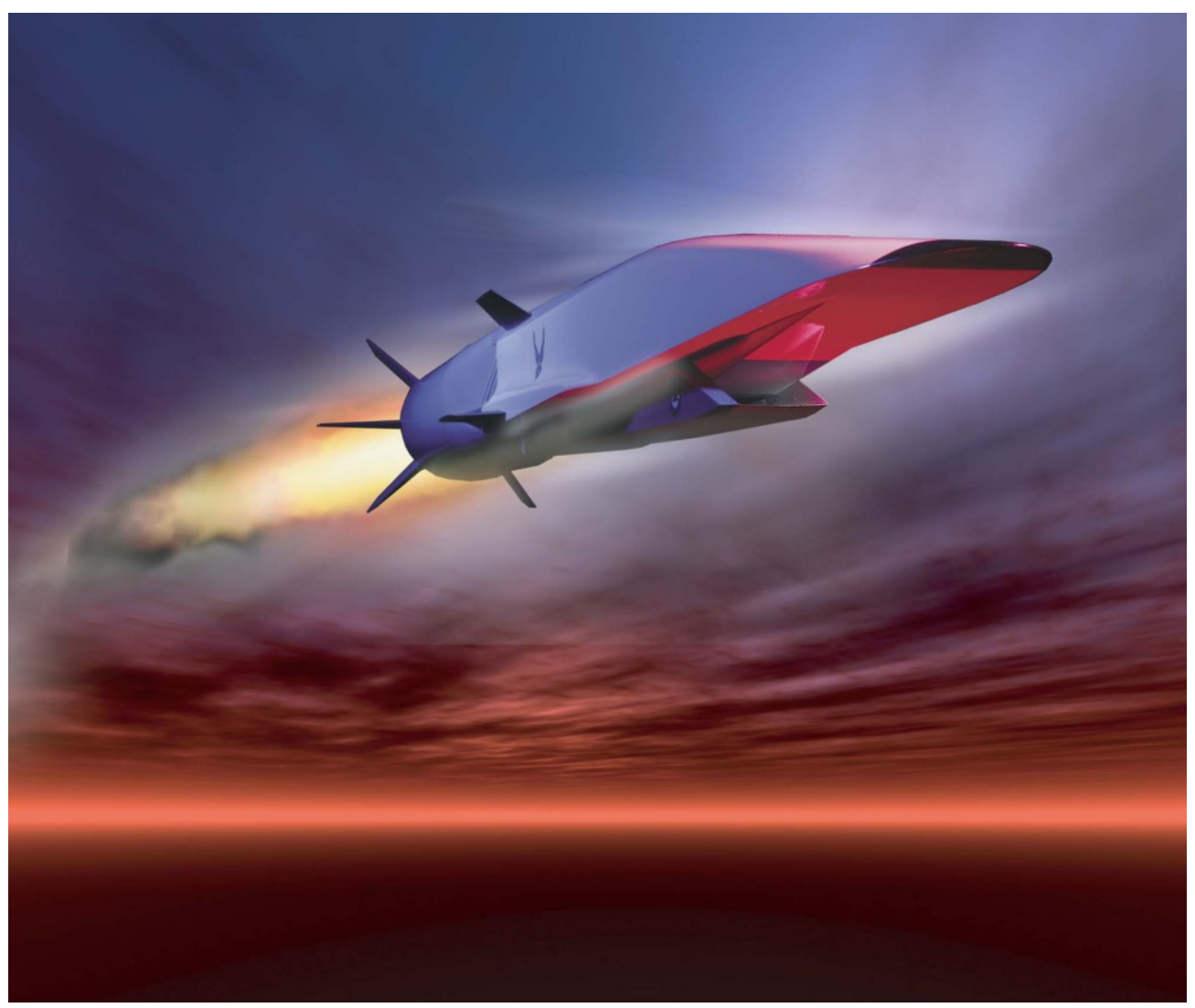

Figure 1: A rendering of a hypersonic vehicle design called the "X-51A Waverider" designed by the U.S. Air Force "public domain"1.

The pursuit of commercial hypersonic flight relies on innovative reusable materials with improved thermal protection systems (TPS) ${ }^{2,3}$. Designs of hypersonic vehicles that are capable of sustained hypersonic flight (Figure 1), coupled with traditional maneuverability, must deal with harsh environments concentrated at a several locations on the aircraft ${ }^{4-6}$. Candidate materials must survive harsh conditions, especially upon atmospheric reentry, including dissociative oxidation at surfaces, extreme heat fluxes, temperatures soaring over $3000^{\circ} \mathrm{C}$ and 
vibrational and stagnation pressure loads $3,7,8$. These harsh conditions are concentrated at leading edges, as the vehicles nose and wings and in the propulsion systems. Leading edges experience high stagnation pressures, oxidizing environments, high heating rates and thermal stresses ${ }^{4,6}$. Similarly the propulsion systems experience high convective heat transfer under oxidizing atmospheres generated by fuel combustion ${ }^{4}$. Survival conditions as harsh as this relies on materials will exceptional high temperature properties.

\subsubsection{UHTCs Materials for Hypersonic Environments}

Ultra-high temperature ceramics ${ }^{9}$ (UHTCs) have been identified as the best suited candidates for those extreme conditions. UHTCs are generally defined as having melting temperatures over $3000{ }^{\circ} \mathrm{C} .{ }^{10}$ Combinations of early metal carbides with boron, nitrogen, or carbon make up most of the UHTC materials ${ }^{11}$. Some common UHTCs are $\mathrm{ZrB}_{2}, \mathrm{HfB}_{2}, \mathrm{TiB}_{2}, \mathrm{TaC}, \mathrm{HFC}, \mathrm{ZrC}$, TaN, HfN and $\mathrm{TiN}^{12}$. The high temperature stability of UHTCs makes them useful for high temperature applications, but their other properties such as oxidation resistance, density and thermal conductivity specify them for high temperature applications. Diborides are of particular interest when designing hypersonic components as they are the most oxidation resistant materials among the UHTCs. Oxidation is a chemical change in the material due to the presence of oxygen and high temperature, often resulting in mass loss. Diborides are typically considered for leading edge applications because of their increased oxidation resistance, while carbides and nitrides are studied for uses in propulsion liners and systems for their high conductivities and mechanical strength ${ }^{5,11,13}$. Diboride materials are designed to distribute the massive heat and mechanical loads from the leading edge across the airframe, while keeping their 
geometries, attributes and features stable to ensure the survivability and reusability of the vehicles and aircrafts.

\section{$\underline{0.1 .3}$ Titanium Diboride}

$\mathrm{TiB}_{2}$ has been proposed as a candidate for applications in hypersonic environments because of its mechanical properties at high temperatures and because it has one of the lowest densities among UHTCs ${ }^{11,14}$. $\mathrm{TiB}_{2}$ shows comparable properties to other UHTC materials at a lower density, shown in Table 1 from Golla et $\mathrm{al}^{10}$. $\mathrm{TiB}_{2}$ was identified as a strong candidate material for hypersonic flight along with the other diborides back in the 1950s, but has not received a tremendous amount of attention because difficulties during manufacturing and densification ${ }^{15}$. There has been recent work to solve the issues with densification ${ }^{16}$, and several studies have reported near full densities ${ }^{10}$.

Table 1: Summary of UHTC properties at room temperature (RT) ${ }^{10}$.

\begin{tabular}{|l|l|l|l|}
\hline Material & $\mathrm{TiB}_{2}$ & $\mathrm{ZrB}_{2}$ & $\mathrm{HfB}_{2}$ \\
\hline Crystal Structure & $\mathrm{Hex}$ & $\mathrm{Hex}$ & $\mathrm{Hex}$ \\
\hline Melting Temperature ${ }^{\circ} \boldsymbol{C}$ & 3225 & 3200 & 3380 \\
\hline Density $\boldsymbol{g} \mathbf{~ c m}^{-\mathbf{3}}$ & 4.5 & 6.1 & 11.2 \\
\hline $\begin{array}{l}\text { Co-efficient of Thermal Expansion } \\
\boldsymbol{\alpha} ; \mathbf{1 0}^{-\mathbf{6}} \boldsymbol{K}^{-\mathbf{1}}\end{array}$ & 7.4 & 6.8 & \\
\hline Thermal Conductivity $\boldsymbol{W} \boldsymbol{m}^{-\mathbf{1}} \boldsymbol{s}^{\mathbf{1}}$ & $60-120$ & $60-105$ & 6.3 \\
\hline Elastic Modulus $\boldsymbol{G P a}$ & 560 & 350 & 104 \\
\hline Hardness $\boldsymbol{G P a}$ & $25-35$ & $20-25$ & 480 \\
\hline Fracture Toughness $\boldsymbol{M P a} \boldsymbol{m}^{\mathbf{1 / 2}}$ & $4-5$ & 4 & 28 \\
\hline & $700-1000(3-$ & & 4 \\
\hline Flexural Strength $\boldsymbol{M P a}$ & point) & $300-400$ (4-point) & $350-450$ (4-point) \\
\hline Oxidation Resistance ${ }^{\circ} \boldsymbol{C}$ & $<1200$ & $<1400$ & $<1400$ \\
\hline
\end{tabular}




\subsubsection{Challenges Facing the Application of UHTCs in Hypersonic Environments}

Although UHTCs are exceptional candidates, there are still many challenges to the application of UHTCs in hypersonic systems, most notably specific strength (strength to density ratio), thermal shock resistance and oxidation at below melt temperatures ${ }^{6}$. Diborides show oxidation resistance up to $1700^{\circ} \mathrm{C}$, and additives of $\mathrm{SiC}$ or $\mathrm{MoSi}_{2}$ have been shown to increased oxidation resistence ${ }^{11}$. Specific strength becomes important when designing aircraft for fuel efficiency and reuse. Thermal shock resistance (TSR) refers to the failure of a material when mechanical stresses appear due to differences in the coefficient of thermal expansion (CTE) and varying temperature. UHTCs follow traditional brittle ceramic behavior and can fail from thermal stresses; this issue is often solved by adding carbon fiber reinforcement into a diboride matrix $^{17,18}$.

Hypersonic flight systems deal with high heat loads above $1000 \mathrm{~W} \mathrm{~cm}^{-2}$ and, in the case of leading edges must functionally dissipate heat to increase long term survivability $2,7,9$. In the past, ITTPs have been designed using isotropic insulators ${ }^{2}$, however the efficient dissipation of high heat loads demand anisotropic insulating structures. This functional design could increase heat flow away from the high load areas while preventing heat flow into temperature sensitive structure components ${ }^{19-21}$. This type of anisotropy can have use in both active and passive cooling packages. Active cooling is where a coolant is used to flow across a porous matrix, in order to dissipate heat away from the high load areas, and passive cooling refers structures used as either a heat sink to direct heat or insulation to prevent heat flow ${ }^{9}$. The use of UHTCs as the main material for these designs will allow the component to handle much higher heat fluxes 
as well as ensuring a smooth integration with other UHTCs parts from the aircrafts. However, there is limited research into anisotropic materials, in the UHTC community.

UHTC's microstructure or chemical structure can be altered to improve properties. One microstructural strategy for combating the issues of specific strength and thermal shock has been to use porous materials ${ }^{22}$. Dense materials still need to be used on the leading edge, but gradient structures with dense and porous material combinations can be used to increase the survivability of functional components. These complex structures are referred to as integrated thermal protection systems (ITTPs) ${ }^{23}$. Research has shown that strength to weight ratios can be controlled with carefully tailored porous structures, creating anisotropic high performance engineered materials ${ }^{24-26}$. Anisotropic porous UHTC's are mainly limited to aligned porous materials from freeze casting ${ }^{10,22}$. Regarding chemical changes to change in properties, doping UHTCs has been shown in some cases to improve properties. Liu et al showed that an addition of 5 vol \% WC into a $\mathrm{HfB}_{2}-\mathrm{SiC}$ matrix increased the flexural strength at $1600{ }^{\circ} \mathrm{C}$ by $57 \%{ }^{27}$. The same group studied a 5 vol \% addition of WC in a $\mathrm{ZrB}_{2}-\mathrm{SiC}$ matrix and only saw a slight increase in behavior at $1600^{\circ} \mathrm{C}$ with the WC showing a cleaner grain structure, authored by Ma et al ${ }^{28}$. Zapata-Solvas et al increased the oxidation performance of $\mathrm{ZrB}_{2}-\mathrm{SiC}$ and $\mathrm{HrB}_{2}-\mathrm{SiC}$ composites at $1600{ }^{\circ} \mathrm{C}$ with $\mathrm{La}_{2} \mathrm{O}_{3}$ doping, the caused the formation of a more stable $\mathrm{MeO}_{x} \mathrm{C}_{y}$ phase, where Me is $\mathrm{Hf}, \mathrm{Zr}$, or $\mathrm{Si}^{29}$. 


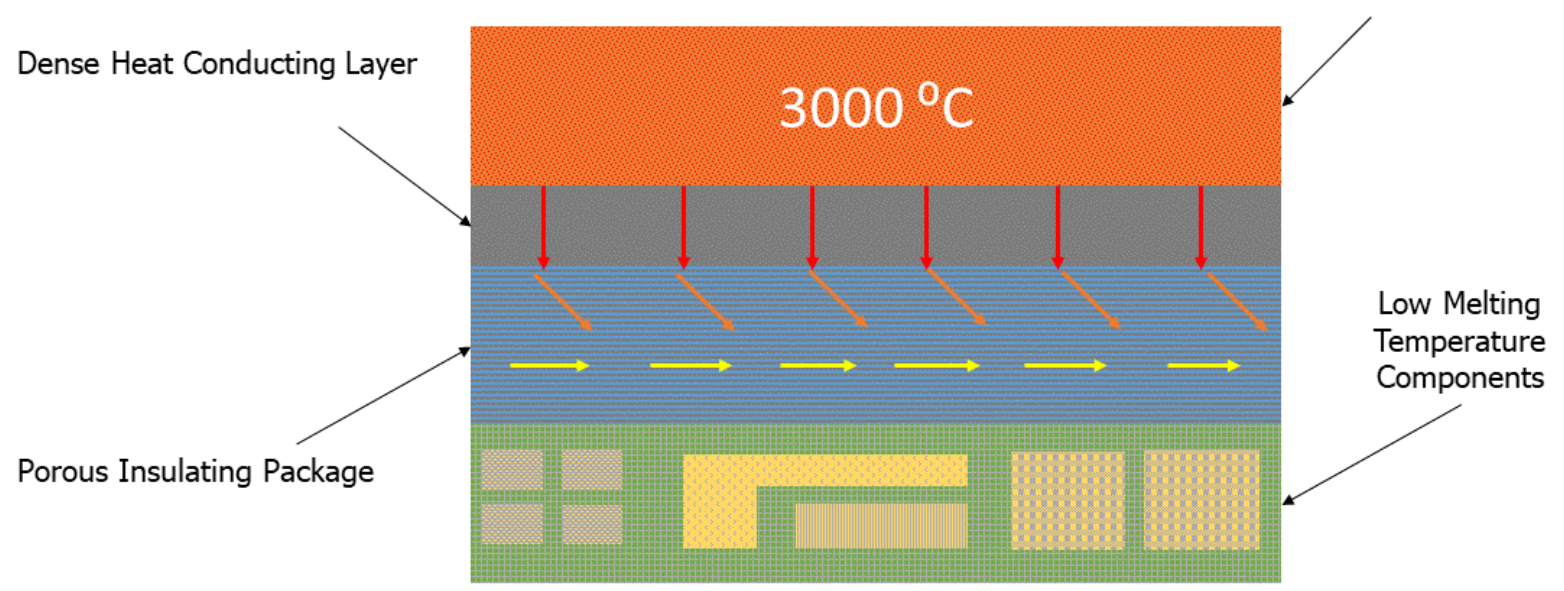

Figure 2: Schematic of a porous insulation layer's role is distributing heat away from internal components.

Figure 2 shows an aligned porous structure in an active cooling application. This form on thermal anisotropic behavior can be accomplished by functionally graded porosities or by the integration of aligned continuous pores. The aligned continuous pores allow for un-interrupted heat conduction in the parallel direction of the pores, while constantly inhibiting heat conduction in the perpendicular direction across the pores. When the aligned pores are continuous, open, and larger than $10 \mu \mathrm{m}$ they can work with a fluid as a heat exchanger to create an active cooling concept through microfluidics ${ }^{30,31}$. Alternatively, the open aligned pores would support a transpirational cooling system, where a coolant is pushed through the pores into the mainstream flow to form a protective layer ${ }^{32}$.

\subsubsection{Thermal Conductivity and Influence on UHTC Performance}

Thermal conductivity is the ability of a materials to transport heat across temperature gradients $^{33}$. Thermal conductivity at RT is comprised of lattice vibrations (phonons) and free 
electrons which transfer the kinetic energy of high temperature areas to low temperature areas. The bond structure in ceramics often has fewer free electrons then metals, and so thermal conduction in ceramics is primarily through phonon transport. However, diboride have a covalent bond structure allowing for a more metal-like conductivity through electrons. At low temperatures thermal conduction is primarily limited by phonon hindering interactions such as crystal defects, grain boundaries, impurities and phonon-phonon interactions ${ }^{34}$. As temperature increases the frequency of phonon-phonon interactions increase until the Debye temperature, where the increased number of phonon interactions work destructively to hinder thermal conduction. The Debye temperature for poly-crystalline $\mathrm{TiB}_{2}$ is roughly $1480{ }^{\circ} \mathrm{C}^{35}$. Thermal conductivity is governed by a material's solid-state properties, but can be changed by changing a materials microstructure, such as the introduction of pores.

The precise effect of composite materials, and specifically porosity on thermal conductivity, is still an area of dynamic research ${ }^{36}$. The most basic way to estimate thermal conductivity of anisotropic materials is to use a series or parallel models that treats thermal conduction similar to electrical conduction ${ }^{21}$. More complex models are being constructed using finite element modeling and even machine learning is currently being used to identify the exact variables that contribute to appropriately estimated thermal properties ${ }^{20,37}$. The research area of thermal modeling has not yet matured to the point of highly accurate singular methods for estimating conductivity across several porous systems, due to limitations in capturing the actual porous component structure and to generate experimental data to validate the model especially under extreme conditions. It is important going forward for all research to carefully and precisely characterize the structure property relationships for UHTC's so a broad encompassing 
understanding can be created for benefit of the materials science and aerospace design communities.

As previously mentioned, thermal conductivity is intrinsically connected to all the challenges in UHTC performance $9,11,38,39$. Thermal conductivity will control the temperature distribution from the leading edge, influencing the oxidation, thermal shock, and mechanical performance. Low thermal conductivities will induce large gradients in temperature and increase the chances of thermal shock. If heat can be dissipated quickly with a high thermal conductivity, both surface and internal temperature will be lower. A lower surface temperature will decrease oxidation. Mechanical properties are often temperature dependent and trends will differ from material to material.

\section{$\underline{0.1 .6}$ Porous UHTC Properties and Characterization}

Porous microstructures are an attractive solution to solve issues of specific strength and anisotropic thermal properties, but the question of the precise effect of pores on thermal conductivity and mechanical strength comes into focus. The aspects generally understood to be improved by a controlled introduction of porosity are thermal shock resistance and insulative thermal conductivity, while mechanical strength will degrade.

A common deficiency in dense UHTCs is thermal shock resistance (TSR) will increase generally with increasing porosity, due to a decrease in young's modulus ${ }^{40}$. Failure due to thermal shock occurs when uneven temperature gradients within a material cause stress resulting in mechanical failure ${ }^{33}$. Thermal shock resistance is increased in porous materials because the porosity results in more ductile behavior and the porosity promotes a smooth thermal gradient 
or reduces crack fatal propagation ${ }^{33}$. When porosity, in highly conducting materials, increases the thermal conductivity always decreases. The upper bound for thermal conductivity of porous ceramics is a rule of mixtures based on the conductivities of the pore gas and the material ${ }^{41}$. Mechanical strength will decrease with increasing porosity due to stress concentrations from the pores, and a reduced solid area to handle the load. Wang et al modeled the effect of porosity volume on $\mathrm{TSR}$ on $\mathrm{ZrB}_{2}$ and estimated a $117 \%$ increase in TSR at $1500{ }^{\circ} \mathrm{C}$ between 5 and $30 \mathrm{Vol} \%$ prosity $^{42}$. Yuan et al studied thermal conductivity as a function of porosity in $\mathrm{ZrB}_{2}$ and saw a reduction in thermal conductivity from 49 to $35 \mathrm{~W} \mathrm{~m}^{-1} \mathrm{~K}^{-1}$ from fully dense to 38 $\mathrm{Vol} \%$ porosity at $800^{\circ} \mathrm{C}$. Studart et al complied a plot of porosity vs compressive strength for alumina and show a decrease in compressive strength from 134-0.18 $\mathrm{MPa}$ at 54 and $96 \mathrm{vol} \%$ porosity respectively ${ }^{43}$. To understand these effects, we will study our manufactured materials properties in controlled environments via mechanical testing, laser flash diffusivity and in the dynamic environment created by an oxy-acetylene torch. Thermal shock will be discussed only semi quantitatively through the rapid heating rates associated with oxy-acetylene testing ${ }^{44-51}$.

\section{$\underline{0.1 .7}$ UHTC Testing in Relevant Environments}

The testing of specific UHTC properties in controlled environments provides valuable information for establishing a processing structure and properties relationship, but these materials must be tested in relevant conditions to truly gauge their functionality. Plasma wind tunnel, arc-jet and scramjet testing are the most accurate methods for approximating hypersonic conditions, but have a high cost per test and are often inaccessible to be used for initial screening of $\mathrm{UHTCs}^{50}$. A cheaper more accessible alternative to these methods is oxyacetylene torch testing ${ }^{44}$. Through the variables of gas ratio, flow rate and distance of the torch 
to the sample, a variety of heat fluxes and oxidizing conditions can be simulated, including heat fluxes as high as $17 \mathrm{MWm}^{-250}$. Traditionally an oxyacetylene test has only been a pass/fail test, where temperature measurements are taken with thermal imaging, or pyrometers and thermocouples to track the test conditions. However, with some modification, oxyacetylene torch testing can be used to extract some key properties for UHTCs, such as the thermal conductivity, mentioned before.

\subsubsection{Processing Routes to Anisotropic Porous Structures}

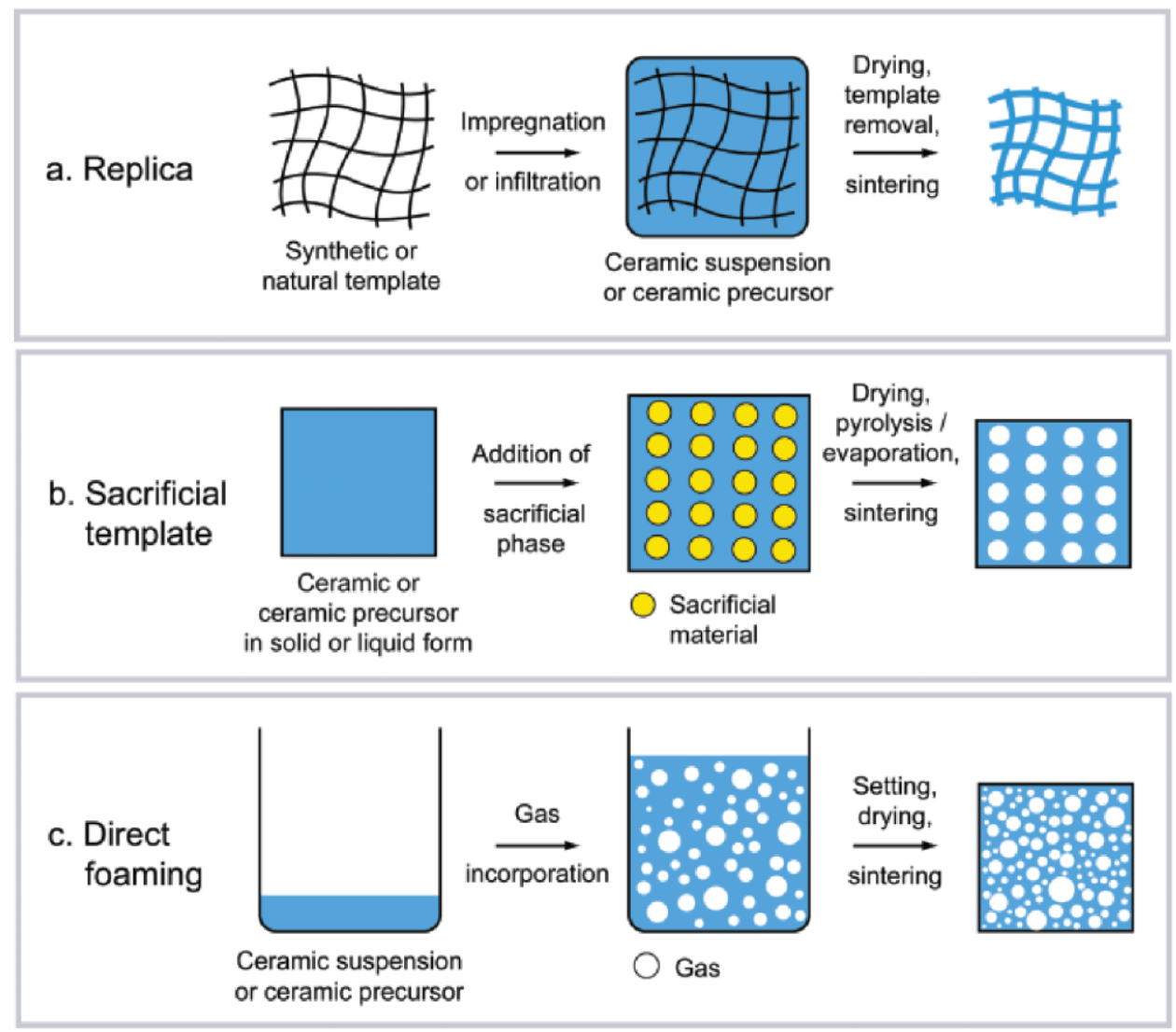

Figure 3: Scheme of popular techniques for producing porous ceramics "reuse with permission" 52 .

Macroporous ceramics can be made through several general processing routes ${ }^{53}$. Figure 3

shows a schematic for several of these techniques. Porous processing that follow colloidal 
routes can be applied to multiple ceramic systems. Most of the routes discussed earlier are commonly done with non-UHTC materials, but work is being done to apply these techniques to UHTC materials. Freeze casting, direct foaming and sacrificial template techniques have been applied to $\mathrm{TiB}_{2}$ and $\mathrm{ZrB}_{2}$ to form complex shapes ${ }^{22}$.

Replica techniques mimic a structured material by infiltrating with a ceramic suspension or chemical converting the structure to a ceramic. $\mathrm{ZrB}_{2}$ sponges have been produced from natural sponges and reticulated polyurethane sponges with porosities ranging from 70-90 vol\% porosity ${ }^{54}$. Herzog et al did the later by taking a pine wood template that was pyrolyzed, infiltrated with $\mathrm{SiO}_{2}$ nano-sol and converted to $\mathrm{SiC}$ through carbothermal reduction to form a structure resembling the wood template ${ }^{55}$.

Sacrificial templating and sacrificial fillers cover several techniques, as structure and porosity can differ greatly based on the template. Polymer beads have been organized through processing with a centrifuge to form organized porous structure in $\mathrm{TiO}_{2}$ and $\mathrm{ZrO}_{2}$ with $70 \mathrm{vol} \%$ porosity ${ }^{56}$.

Direct foaming is a popular technique where the chemistry of a ceramic suspension is controlled to form a stable foam through the incorporation of air bubbles ${ }^{57}$. Chuanuwatanakul et al produced large complex shapes with cross-linked particle stabilized foams from alumina with close to $90 \mathrm{vol} \%$ porosity showing green bodies strong enough for light machining ${ }^{58}$. 
Ice templating creates a porous material by freezing a colloidal suspension causing separation between the frozen solvent and ceramic particles. Icetemplaing has been used to make an aligned porous structure of alumina with different pore sizes and pore wall thicknesses ${ }^{25}$.

Ceramics can be organized on the microporous level through preceramic block copolymers (BCP). Malenfant et al synthesized polynorbornene-block-polynorbornenedecaborane (PNB-bPDB), organized the BCP through solvent annealing with THF, and pyrolyzed to form an organized boron nitride structure ${ }^{59}$.

Extrusion or co-extrusion repeatedly extrudes an organized assembly of ceramics and sacrificial fillers through a nozzle to create a highly organized array of pores. Isobe used extrusion to organize polymer rods in alumina, producing an anisotropic material with cylindrical pores of aspect ratio $40^{60}$.

Partial sintering is the most common and convenient technique for producing porous structures for all ceramic materials ${ }^{61}$. Partial sintering is a process by which a material is not fully densified during sintering such that a controllable amount of porosity is left between particles. The porosity and pore size depending on sintering parameters and particle size. Alumina with porosity ranging from $30-45 \mathrm{vol} \%$ has been made by changing the sintering temperature between 1100 and $1400{ }^{\circ} \mathrm{C}^{61}$.

We plan to apply a sacrificial filler technique because of the diverse options in filler type, additionally we will use partial sintering to generate multi-scale porosity to better control thermal conductivity and reduce weight. 


\section{$\underline{0.1 .9}$ Electrospinning as a New Sacrificial Template}

Sacrificial template techniques are the most promising techniques to achieve complex and highly organized porous structures. The degree of organization of a sacrificial filler often translates to that of the final porous microstructure. For example, replica ceramics made from reticulated poly-urethane sponges directly follow the structure of the sponge, but extra effort must go into orienting polymer rods to form an organized structure ${ }^{54}$. Electrospinning allows for the orientation of a sacrificial filler into an array of organized structures. Electrospinning is a process where a polymer solution is ejected from a millimeter size needle and accelerated towards a conductive collector by an electric field. The electric field causes bending instability, stretching the fiber and decreasing the diameter as the solvent evaporates. Several parameters can be used to control the fiber diameter polymer solution viscosity, electric field magnitude, solution ejection rate, and collector distance ${ }^{62}$.

Aligned fibers meshes, with simultaneous spinning of multiple diameters and fiber yarns can all be produced with modified electrospinning apparatuses ${ }^{62}$. Because electrospining utilizes polymer solutions, a wide array of fiber chemistries can be employed. Block-copolymer fibers, porous fibers, and fibers composed of ceramic precursors have all been processed with electrospinning ${ }^{62}$. Electrospun fibers can be used as the sacrificial filler, because of their high aspect ratio, uniformity, precisely controllable diameter over a wide size range ( $\sim 50 \mathrm{~nm}-50 \mu \mathrm{m})$ and their defined geometry from the spinning process. 


\section{$\underline{0.1 .10}$ Conclusion}

All of these attributes will translate into highly oriented porous structures, compatible with the creation of anisotropic UHTC materials to control thermal conductivity and mechanical properties to be used in active cooling applications. The aligned open pores derived from the electrospun fibers combined with partial sintering of $\mathrm{TiB}_{2}$ will create a multiscale porous structure where thermal and mechanical properties can be optimized. Anisotropy from the aligned pores will create a structure suitable for passive cooling, and the open nature of the pores will allow for active cooling in the future. 


\section{Objective}

In this work we propose a new and simple method to produce anisotropic multi-scale porous ceramics with aligned continuous pores suitable for microfluidics regime in active cooling components. A classical sacrificial filler approach will be taken with an innovative sacrificial filler of electrospun polystyrene (PS) fibers. The removal of the PS fibers will create aligned continuous pores that run the whole length of the ceramic part. The porosity derived from the sacrificial filler combines with the small inter-particle porosity generated from the sintering process, creating a multiscale porous ceramic that can target the requirements of lightweight, thermal conductivity and mechanical integrity suitable for a UHTC active cooling component.

The anisotropic $\mathrm{TiB}_{2}$ material from this new processing method will be characterized to understand the structure properties relationship, and compared to current anisotropic icetemplated $\mathrm{TiB}_{2}$. Thermal conductivity will be measured in the pore direction over a range of temperatures. Compressive strength will be measured in the pore direction, at RT. By taking common UHTC measurements we will be able to compare our technique to other porous structures.

To improve the understanding of these materials at higher temperatures and under oxidizing conditions, an improved oxyacetylene test will be modeled. By controlling the material heat flow during testing with a sample holder of known materials, a finite difference thermal model and common in situ temperature measurements an approximate thermal conductivity can be measured. 


\section{Chapter 1: Aligned Continuous Cylindrical Pores Derived from Electrospun Polymer Fibers in Titanium Diboride*}

\subsection{Introduction}

There are currently several processing routes to creating aligned continuous cylindrical pores in ceramic materials. Block-copolymer templating, ice-templating, fugitive cotton thread, and extrusion are techniques that produce similar structures to what is need for this type of anisotropic insulation package. ${ }^{52}$ Figure 4 shows an illustration for each of these techniques.

a)

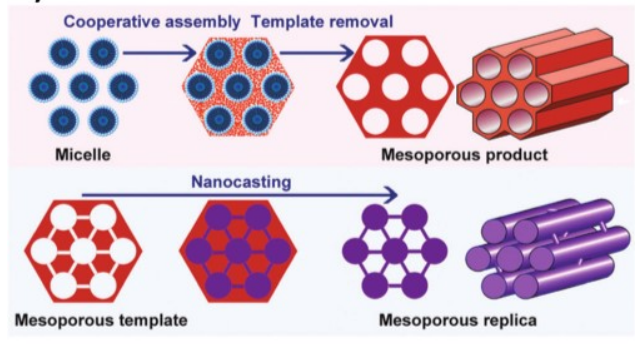

c)

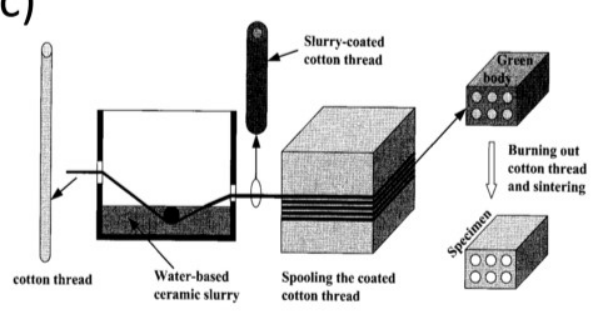

b)

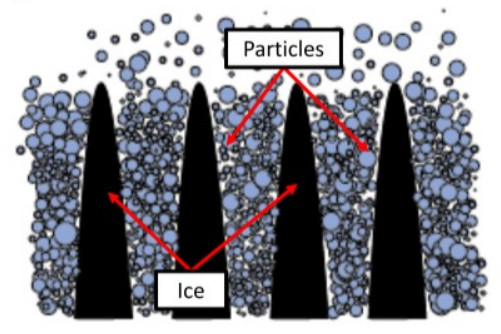

d)

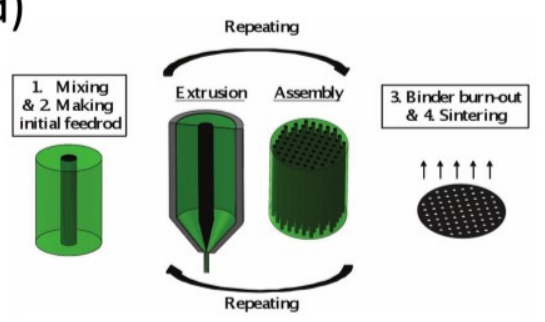

Figure 4: Several methods for producing aligned porous structures a) block copolymer templating, b)icetemplating, c) fugitive cotton thread and d) extrusion, extracted from multiple sources "open access/reuse with permission" ${ }^{63-66}$.

\footnotetext{
* adapted from publication with permission from Hicks DC, Zhou Z, Liu G, Tallon C. Aligned continuous cylindrical pores derived from electrospun polymer fibers in titanium diboride. Int J Appl Ceram Technol. 2018;00:1-12.
} 
Block-copolymer templating uses a polymer template to form a range of highly organized and controlled mesoporous structures. For application in UHTC material materials need to be produced on the scale of $100 \mathrm{~mm}$. BCP templating is hard to scale up both in final material size and pore size to the regime of microfluidics. BCP is focused more or materials with electric applications where high surface area is a priority. Hsueh et al produced a nanostructured $\mathrm{TiO}_{2}$ with surface area $257 \mathrm{~m}^{2} \mathrm{~g}^{-1}$ for uses in photocatalysis ${ }^{67}$. $\mathrm{TiO}_{2}$ with an organized structure was produced by block-copolymer templating with pore walls at $10 \mathrm{~nm}$ near the excitation diffusion length. Block copolymer templating can produce highly organized materials, but cannot produce materials on the size scale necessary for hypersonic insulation application.

Ice-templating makes aligned pores with sizes compatible with microfluidics, but it becomes difficult to have uninterrupted interconnected aligned pores when the parts have complex shapes or large sizes. With ice-templating complex shapes can be casted making it a viable option for insulation materials. Ice-templating has even been used to cast large parts from UHTCs, both $\mathrm{TiB}_{2}$ and $\mathrm{ZrB}_{2}{ }^{22}$. Ice-templating pore organization follows the freezing front and making it difficult to product pores in multiple directions, limiting the complexity of structures possible. The pore morphology has been controlled somewhat more precisely with the addition of magnetic particles to the suspension, but the technique is still limited ${ }^{68}$.

Extrusion techniques are very proficient at making porous networks in one dimension, but have a low limit on pore size and creating 3D complex geometries is not possible. Extrusion has tremendous capacity for controlling material structure. Hoy et al produced ceramics with a features in the shape the capital letter $M$, the symbol for the University of Michigan, down to 
the level of $10 \mu m^{69}$. Extrusion techniques have also been used to make a $\mathrm{ZrO}_{2}$ with negative poison ratio $^{70}$.

Sacrificial filler techniques have potential for organized and diverse pore structures by controlling the orientation of the filler material in a mold. Fugitive cotton thread is based on a sacrificial filler route, where cotton thread is coated in a ceramic slurry and made into a green body ${ }^{65}$. The cotton threads are then burnt out and the resulting structure has pores on the order of $100 \mu \mathrm{m}$. However, these pores have very rough walls that will affect the fluid flow, and the technique has showed restricted versatility towards the casting of complex shapes.

The use of PS electrospun fibers allows us to control the fiber diameter and create mats of fibers with aligned fibers. The aligned fiber mats can be consolidated in molds for an organized sacrificial template. Through electrospinning there is potential for multiple organization structures by spinning two polymer solutions ${ }^{62}$. Before the array of complex geometries are forms the casting technique for combining a ceramic suspension with electrospun PS must be developed. In Chapter 1 we propose a method using vacuum infiltration casting to combine a ceramic suspension and electrospun fibers to form an aligned porous ceramic from a UHTC material.

\subsection{Experimental Section}

\subsubsection{Ceramic Suspension Preparation}

Titanium diboride (TiB2; grade F, purity $\geqslant 96 \%$, dv50=2.9 $\mu m$, H.C. Stark, Germany) was used as the ceramic powder. Ethanol (EtOH; 200 proof, $\geqslant 99.2 \%$, Decon Labs, USA) was selected as 
the solvent and Dolapix CE 64 (Zschimmer and Schwartz, USA) as the dispersant for making the ceramic suspension.

Ceramic suspensions with solid loadings between 20 and 40 vol\% were prepared using ethanol as a solvent and $0.05 w t \%$ of dispersant respect to the weight powder. The suspensions were stirred manually using a spatula until became homogeneous. The mixed suspensions were sonicated for 2 minutes at $40 \mathrm{~Hz}$ (with ultrasound pulses for $1 \mathrm{~s}$ on and $1 \mathrm{~s}$ off) using a sonicator horn (Sonic Dismembrator Ultrasonic Processor, model FB-705, Fisher Scientific, USA) to break up any agglomerates existing in the as-received powder. After that, the suspension was rolled for $30 \mathrm{~min}$ to ensure homogenization before it was used.

\subsubsection{Sacrificial Filler Production}

The PS fibers used as sacrificial fillers in this work were prepared using electrospinning.

Polystyrene (PS; MW 192 k, Sigma Aldrich, USA), N,N-dimethylformamide (DMF; >99.7\%, Sigma Aldrich, USA) and chloroform ( $\geq 99.99 \%$, Fisher Scientific, USA) were used to create polystyrene fibers. PS was chosen as the fiber material because of its availability, low cost and well documented properties $^{71}$. As shown in Figure 5 a, the PS fibers were electrospun on a homemade rotating drum to produce a highly aligned fiber mat, similar to the procedures reported by Fennessey et. al. ${ }^{72}$, and Teo et. al. ${ }^{62}$ The electrospinning setup consisted of several components including a high voltage power supply (model P030HP1, Acopian, USA), a metal drum collector (135 $\mathrm{mm}$ in diameter, $150 \mathrm{~mm}$ in length), a rotator by Southwest Science (Part 
SOS20-S, Southwest Science, USA), and a syringe pump (EW-74905-58, Cole-Parmer, USA).

a)

\section{Electrospining}

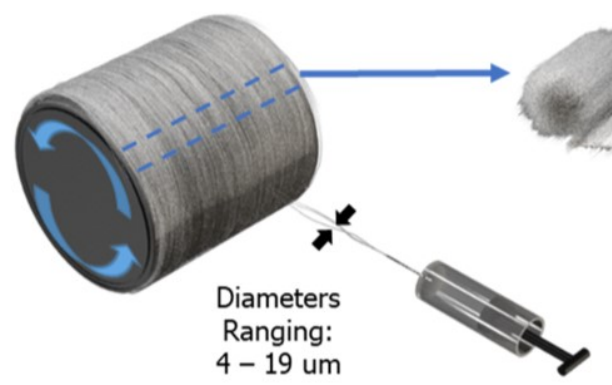

b)

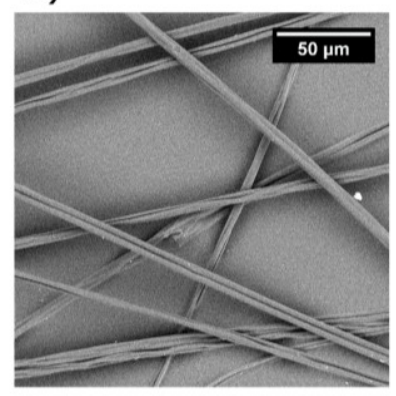

c)

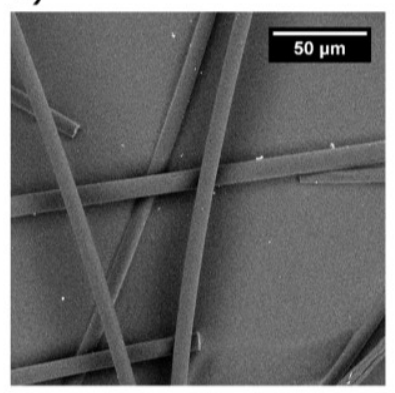

Fiber

Arranging d)

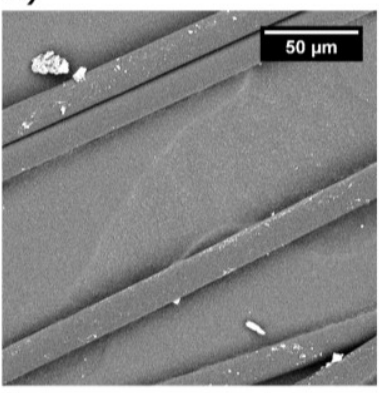

e)

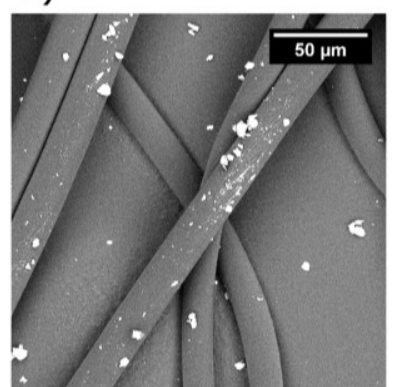

Figure 5: Illustration of the electrospinning set up and fiber preparation of the fiber swatch for placing into the mold; (B, C, D, and E) electrospun polystyrene fibers produced in this work with diameters of $4 \mu \mathrm{m}, 8 \mu \mathrm{m}, 17 \mu \mathrm{m}$, and $19 \mu \mathrm{m}$, respectively. (The bright spots on the fibers are debris derived from the sample mounting for the SEM observations.)

The polystyrene (PS) fibers used as sacrificial fillers in this work were prepared using electrospinning. Polystyrene (PS; MW $192 k$, Sigma Aldrich, USA), N,N-dimethylformamide (DMF; $\geq 99.7 \%$, Sigma Aldrich, USA) and chloroform ( $\geq 99.99 \%$, Fisher Scientific, USA) were used to create PS fibers. As shown in Figure 5a, the PS fibers were electrospun on a custom-built electrospinning setup, similar to those reported by Fennessey et. al. ${ }^{72}$, and Teo et. al. ${ }^{62}$ The custom-built electrospinning setup consisted of several components including a high voltage power supply (model P030HP1, Acopian, USA), a rotating metal drum collector (135 mm in diameter, $150 \mathrm{~mm}$ in length) to produce a highly aligned fiber mat, a rotator by Southwest Science (SOS20-S, Southwest Science, USA), and a syringe pump (EW-74905-58, Cole-Parmer, 
USA). The diameter of the resulting fibers was controlled by the flow rate and concentration of polymer solution out of the syringe. Concentration was controlled by changing the $w t \%$ of PS in DMF between 29 and $44 \%$ of total solution weight. Chloroform was added in small concentrations, 2-3 $w t \%$, to ensure the fiber dried before being deposited on the drum. Flow rate was varied between 0.2 and $0.3 \mathrm{ml} / \mathrm{min}$. Voltage, collector rotation, and throw distance between the syringe needle tip and the fiber collector were all kept constant at $18 \mathrm{kV}, 600$ $\mathrm{rpm}$, and $22 \mathrm{~mm}$ respectively. These parameters were adjusted to create four different fiber diameters $(4,8,17$ and $19 \mu \mathrm{m})$. Individual PS fibers produced in this work are shown in Figure 5, and their associated spinning parameters are summarized in Table 2. The fabricated PS fiber mats were $150 \mathrm{~mm}$ by $420 \mathrm{~mm}$. The density of the PS fibers was measured with a helium gas pycnometer (model ACCUPYC II 1340, Micrometrics, USA) and the value was centered around $1.09 \mathrm{~g} / \mathrm{ml} \pm 2 \%$, which is close to the theoretical density of PS $1.04 \mathrm{~g} / \mathrm{ml}^{73}$.

Table 2: Summary of electrospinning parameters to produce our sacrificial filler fibers.

\begin{tabular}{|c|c|c|c|c|}
\hline Diameter $(\boldsymbol{\mu m})$ & PS $(\boldsymbol{w t} \%)$ & DMF $(\boldsymbol{w t} \%)$ & $\begin{array}{c}\text { Chloroform } \\
(\boldsymbol{w t} \%)\end{array}$ & $\begin{array}{c}\text { Flow Rate } \\
(\boldsymbol{m l} / \mathbf{m i n})\end{array}$ \\
\hline 4 & 22 & 72 & 6 & 0.06 \\
\hline 8 & 27 & 67 & 6 & 0.12 \\
\hline 17 & 26 & 64 & 10 & 0.2 \\
\hline 19 & 28 & 63 & 9 & 0.2 \\
\hline
\end{tabular}

\subsubsection{Vacuum Infiltration Casting (VIC) Rig}

A vacuum infiltration casting (VIC) rig was constructed by modifying the classical vacuum flask with a stopper set up, as shown in Figure 6. The customized rig comprised of several components: a faucet aspirator vacuum pump (part number 312635, Dynalon Labware, USA), a 
liquid flow meter (1/2" NPS Threaded, Adafruit, USA), and a vacuum gauge (0-100 $\mathrm{kPa}$ range, 23\% accuracy, Winters Instruments, USA). Cylindrical molds for casting were made by cutting 22 $\mathrm{mm}$ sections from clear vinyl tubing with an internal diameter of $8 \mathrm{~mm}$. The molds were placed within the stopper. A piece of wire mesh with $1 \mathrm{~mm}$ mesh spacing was below the mold. A filter paper was placed on top of the wire mesh to prevent the bulk of PS fibers from pulling through the mold. The aspirator pulled vacuum at a range of different fluxes corresponding to a water flow between 4 and $10 \mathrm{l} / \mathrm{min}$, and reached vacuum pressures up to $-75 \mathrm{kPa}$. A large diameter pipe with an internal diameter of $8 \mathrm{~mm}$ and length of $70 \mathrm{~mm}$ was fitted on top as a reservoir to hold up to $4 \mathrm{ml}$ suspension for the casting process.

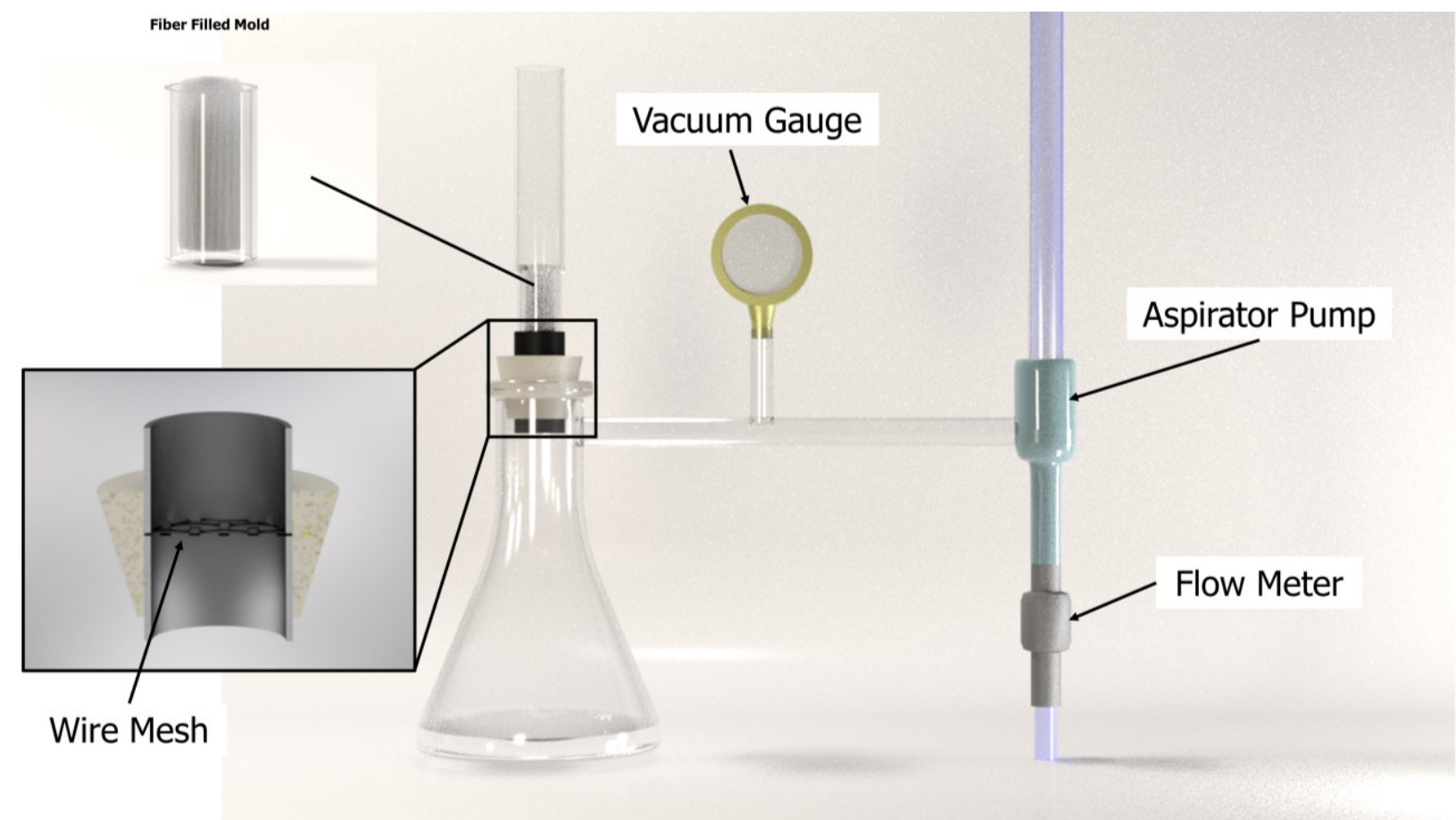

Figure 6: Vacuum Infiltration Casting (VIC) Rig designed for casting electrospun fiber filled molds and produced green bodies. 


\subsubsection{Casting of green bodies}

Pore size and porosity was varied by choosing fibers with different diameters while keeping constant the total amount of fibers at $20 \mathrm{vol} \%$ respect to the mold volume. Additionally, the effect of fiber amount was explored for a fixed fiber diameter $(17 \mu \mathrm{m})$, using amounts of fibers in the mold of 10, 15 and $20 \mathrm{vol} \%$ with respect to the mold volume. The clear vinyl mold was coated with demolding grease (Krytox, GPL 205, Chemours, USA), and then weighed. Then, a section (swatch) of the aligned fiber mats was cut the same width as the mold (as depicted with dashed lines in Figure 1 a). The swatch of fibers was weighed and sections were cut from the length until it matched the desired weight. The cut fiber mat was rolled to form a cylinder that fitted within the mold shown in Figure 6. The filled mold was then weighed to corroborate the precise amount of fibers. The mold was fitted in the VIC, as described in section 2.4. The suspension (whose concentration was selected based on the fiber diameter and amount) was placed in the reservoir. An initial flow rate for the aspirator pump to control the vacuum was then selected. Once the suspension filled the mold, the vacuum was lowered to maintain a value of $-5 \mathrm{kPa}$ and then slowly raised until the maximum vacuum of the aspirator pump. The casting conditions required an initial increase in vacuum to ensure the entire mold filled. The parameters for casting each sample are shown in Table 3. The wet green bodies were then removed from the VIC and allowed to dry at RT for $24 h$. Dried green samples were weighed to estimate if the coverage of the fibers was correct and evaluate the presence of potential processing flaws. 
Table 3: Summary of the Vacuum Infiltration Casting (VIC) proccesing methof parameters.

\begin{tabular}{|c|c|c|c|c|c|c|}
\hline $\begin{array}{l}\text { Fiber Diameter } \\
\qquad(\mu m)\end{array}$ & $\begin{array}{c}\text { PS } \\
(\text { vol \%) }\end{array}$ & $\begin{array}{c}\mathrm{TiB}_{2} \\
\text { suspension } \\
(\text { vol } \%)\end{array}$ & $\begin{array}{c}\text { Aspirator } \\
\text { Flow (L/ } \\
\text { min) }\end{array}$ & $\begin{array}{c}\text { Max } \\
\text { Vacuum } \\
(\boldsymbol{k P a})\end{array}$ & $\begin{array}{l}\text { Pore Size } \\
\qquad(\mu m)\end{array}$ & $\begin{array}{l}\text { Porosity } \\
\text { (vol \%) }\end{array}$ \\
\hline $4 \pm 1.6 \%$ & 20 & 20 & 10 & -16 & 6 & 56 \\
\hline $8 \pm 1.0 \%$ & 20 & 20 & 7.5 & -18 & 8 & 49 \\
\hline \multirow{3}{*}{$17 \pm 1.6 \%$} & 20 & 30 & 9.2 & -22 & 15 & 72 \\
\hline & 15 & 40 & 5.9 & -21 & 14 & 68 \\
\hline & 10 & 40 & 6 & -8 & 14 & 61 \\
\hline $19 \pm 2.4 \%$ & 20 & 35 & 8 & -17 & 21 & 72 \\
\hline
\end{tabular}

\subsubsection{Sintering}

A vacuum graphite (Red Devil-G, R.D. Webb, USA) furnace was used to remove the PS fibers and carry out the pressureless sintering. The initial part of thermal cycle was conducted in vacuum (0.02 mbar). Thermal gravimetric analysis (TGA) data shows that polystyrene compounds with molecular weights between 110 and $2000 k$ fully degrade at $450 o C^{74}$. A heating rate of 2 ${ }^{\circ} \mathrm{C} / \min$ until $500^{\circ} \mathrm{C}$, in vacuum, was used to ensure the gradual removal of the PS sacrificial fillers and to prevent damage to the green body during this PS decomposition. That temperature was maintained for $30 \mathrm{~min}$ to ensure all the PS fibers removed. The samples were then heated at a rate of $5^{\circ} \mathrm{C} / \mathrm{min}$ until $2000^{\circ} \mathrm{C}$, with a dwell time of $1 \mathrm{~h}$. The cooling rate was $5^{\circ} \mathrm{C} / \mathrm{min}$ and the furnace was backfilled with argon above $1800^{\circ} \mathrm{C}$.

\subsubsection{Characterization}

The fibers, powders, fresh fractures of the green and sintered bodies were observed using a Table Top SEM (Phenom Pro, Model 800-07333, Phenom World, USA). Fiber diameters, pore diameters and inter-pore spacing were measured using ImageJ (version: 1.51j8, Wayne Rasband, National Institute of Health, USA). Viscosity of the suspensions was measured using a 
rheometer (AR-G2, TA Instruments, USA), with a concentric cylinder configuration (rotor diameter of $28 \mathrm{~mm}$, a cup diameter of $30 \mathrm{~mm}$, and a rotor immersed height of $42 \mathrm{~mm}$ ) for shear rates between 10 and $1000 s^{-1}$. The density (and porosity) of the sintered materials were measured using a modified Archimedes' method, where the samples were coated with a thin layer of wax of know density to protect the samples during the immersion in the measurement fluid $^{58}$. The percentage of overall porosity is calculated as $100 \%$ minus the percentage of relative density of the sintered specimens (that is density of the sample measured from Archimedes' method divided by the theoretical density of $\mathrm{TiB}_{2,}$, multiplied by 100$)$.

\subsection{Results and Discussion}

\subsubsection{Effect of Size and Amount of the Electrospun Sacrificial Filler on Porosity}

Figure $5 b$-e show the electrospun fibers with the four different diameters: $4,8,17$, and $19 \mu m$, respectively. The PS fibers were uniform in size and morphology as can be seen from the images in Figure 5 and the standard deviation in Table 2 . The $4 \mu \mathrm{m}$ fibers had some cosmetic issues, such as difference in surface roughness but, as it will be shown later, the morphology and size of the pores were not affected. Some of the fibers showed cut or shorter sections on the SEM images, but this was an artifact of the SEM mount preparation, and not indicative of the final aspect ratio of the fibers. The four different fiber diameters were selected based on the ceramic particle size and the potential application in active cooling components and microfluidic regime ${ }^{31}$. The ratio of fiber diameter to particle size needed to be adequate to ensure the particles arrangement around the fiber can provide sufficient resolution to the cylindrical pores. Fibers with diameter range of 15 to $20 \mu \mathrm{m}$ are more suitable for regime of microfluidics than the smaller fibers, and therefore fibers of 17 and $19 \mu \mathrm{m}$ were chosen in this 
study to demonstrate the viability of this processing technique to produce controlled fiber diameters in that range and the corresponding porous materials with those pore sizes. These two very similar fiber diameters were selected to investigate how sensitive the electrospinning fiber and vacuum infiltration casting techniques are to tailor fiber size and pore size for these applications.

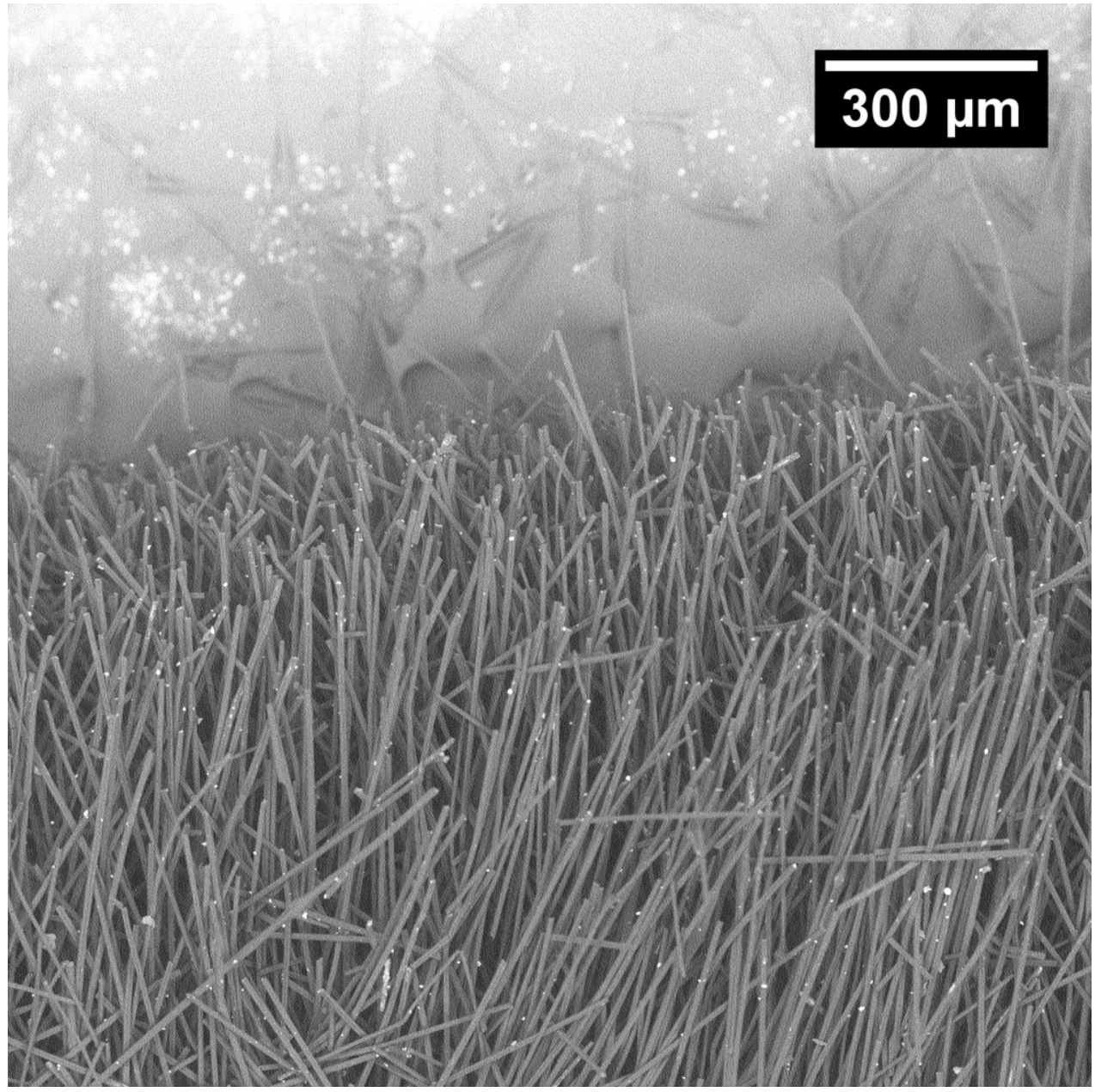

Figure 7: Image of cut end of an aligned PS fiber mat.

The success of a sacrificial filler technique relies heavily on the morphology of the filler material and in our case we rely on the organization of the filler material before casting. It was qualitatively estimated based on experience that the fibers spun here are approximately $70 \%$ 
aligned. Figure 7 shows the cut end of an aligned mat of fibers that would be rolled and placed in a mold. The ends are disturbed during cutting and result in some disorder among the fibers, even so the matt appears to be well organized.

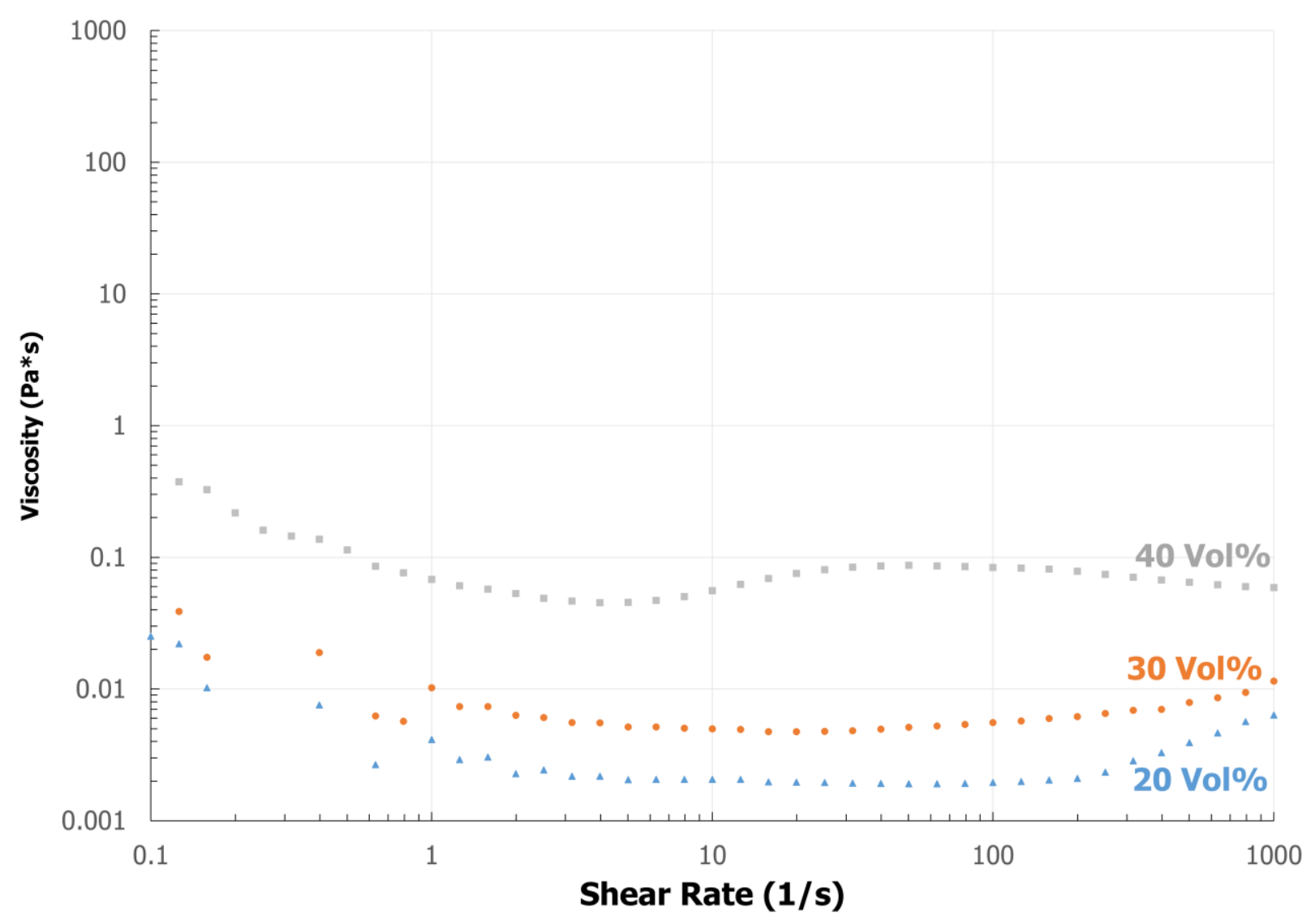

Figure 8: Viscosity vs shear rate curves for suspensions of TiB2 in ethanol with $3 w t \%$ dispersant with respect to powder and varying solid content $(20,30$, and $40 \mathrm{vol} \%$.)

Figure 8 depicts the viscosity behavior of the $\mathrm{TiB}_{2}$ suspensions with three different solid loadings as a function of the shear rate. As expected, the viscosity of the suspensions increased with the $\mathrm{TiB}_{2}$ concentration. All the suspensions exhibited viscosity values below $1 \mathrm{~Pa}$ for all the shear rates measured, with shear-thinning or almost Newtonian behavior. This viscosity 
ensures pourability and a good flow of the ceramic suspension through the fiber-filler mold during the VIC process.

Table 2 shows the processing conditions that enabled the preparation of green bodies with successful coverage of the PS fibers with the ceramic suspensions. In general, as the fiber diameter decreases (for a fixed total amount of fibers) there is more resistance for the suspension to flow through the fiber-filled-mold placed in the $\mathrm{VIC}$ rig, due to the increase in surface area of the fibers. As the fiber diameter decreases, there is more area for interactions between the fibers and suspension, since there is a larger number of fibers in the mold, and the spacing in between them decreases. In order to accommodate this change in the flow resistance trough the fiber-packed mold, the suspension solid loading was varied as $20,30,35$ and $40 \mathrm{vol} \%$. Lower solid concentrations and lower viscosities were more suitable for smaller fiber diameter (that means larger number of fibers per volume, very small spacing between fibers), while higher solid concentrations with a slightly higher viscosity were used for larger fiber diameters (less number of fibers per volume, more space in between fibers). Our results indicate that the solid concentration of the suspensions did not affect the overall porosity of the samples. The porosity of the resulting materials was tuned with the fiber diameter, and the different solid concentration were just used to ensure enough coverage and wetting of the fibers. 
a)

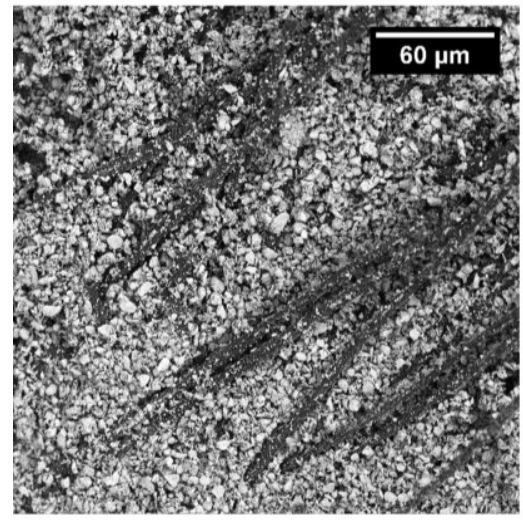

b)

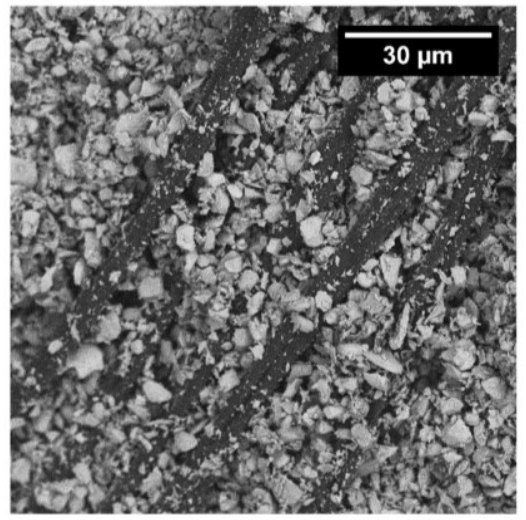

c)

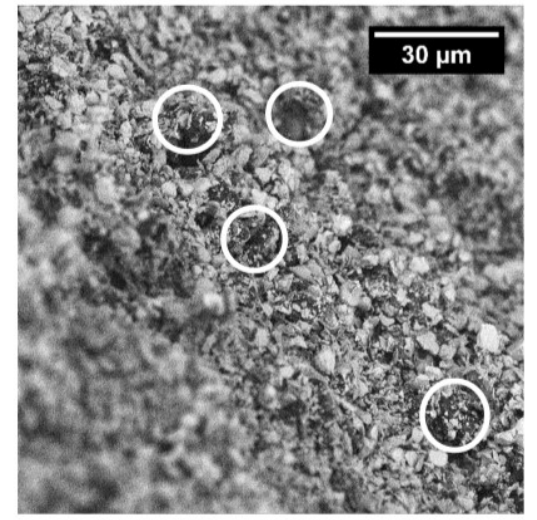

Figure 9: SEM micrographs of fresh-fractured $\mathrm{TiB}_{2}$ green bodies: ( $A$ and $B$ ) parallel cross section to fibers alignment, and (C) perpendicular section to fibers alignment (the white circles have been added to highlight the fiber location.)

Figure 9 shows SEM micrographs of fresh-fractured green bodies. These images show that PS fibers were well dispersed and become fully integrated within the green bodies, keeping the alignment created during the electrospinning process. There are no visible cases of broken fibers in the transverse fracture images. The PS fibers are surrounded by the $\mathrm{TiB}_{2}$ particles in the green bodies, indicating a great affinity of the ceramic suspension for the fibers to produce homogeneous coating. The fugitive cotton thread method reported by Zhang et al. ${ }^{65}$ showed that the slurry was rapidly soaked up by the cotton fibers, and preventing absorption of the ceramic similar to a slip cast system ${ }^{65}$. Sakka et al. used polymer spheres as a sacrificial filler in a suspension, and they controlled the charge and zeta potential difference between the ceramic particles and the filler material to create a system where particles adhere to the filler material ${ }^{56}$. However, in most of the instances where the sacrificial filler material is much larger than the ceramic particles in suspension, there is no specific description of the mechanism of interaction between fillers and particles ${ }^{75,76}$. In the present work, the electrospun PS fibers did not have any pores, and therefore there were no issues with soaking of the fibers, and the 
ceramic particles fully surrounded the fibers, coating them completely without the need for further adjustment of the chemistry of the fibers or ceramic slurry. Further work will be performed to fully understand the mechanism of dispersion of the fibers in the suspension and the wetting of the fibers by the ceramic particles.

a)

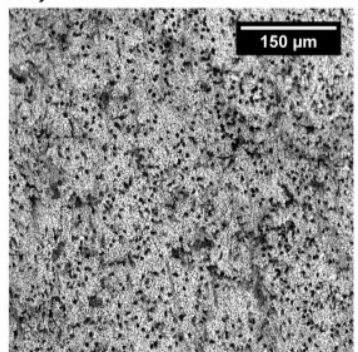

e)

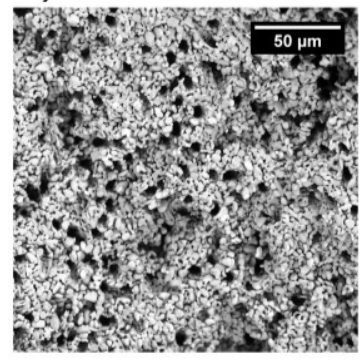

b)

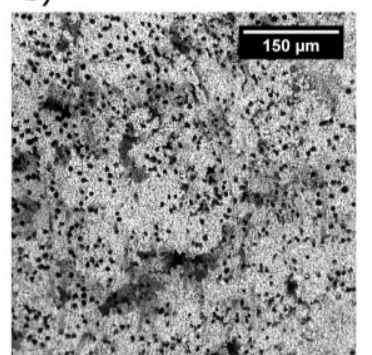

f)

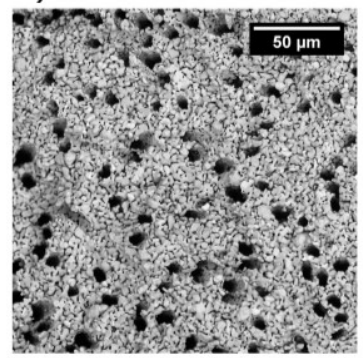

c)

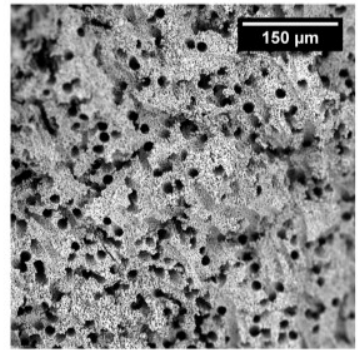

g)

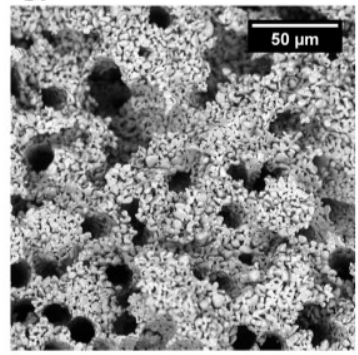

d)

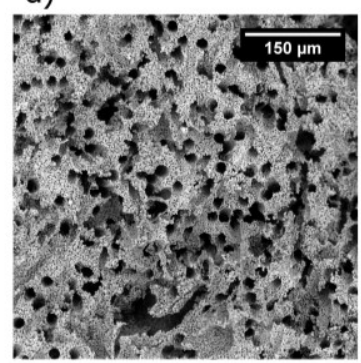

h)

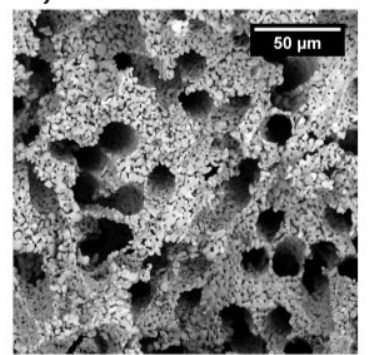

Figure 10: SEM micrographs of fresh-fractured surfaces perpendicular to fiber alignment of sintered $\mathrm{TiB}_{2}$ samples prepared with different fiber diameters: (A-D) low magnification and (E-G) high magnification images of samples prepared with fibers of diameters $4,8,17$, and $19 \mu m$ respectively.

Figure 10 shows SEM images of the sintered multi-scale porous structures prepared with various fiber diameters, taken on fresh-fractured surfaces, at two different magnifications. The total amount of PS fibers for each of the samples with different fiber diameter was the same (20 vol \%). The lower magnification images (Figure $10 \mathrm{a}-\mathrm{d}$ ) indicate a high degree of dispersion of the fibers across the sintered bodies, with relatively even spacing among the pores in each sample. This high degree of dispersion of the pores in the sintered bodies is due to the distribution and dispersion of the fibers in the green body. Apart from the use of PS fibers, no 
other additives were used in the ceramic suspension. The use of EtOH as the suspension solvent has played a significant role in the fiber dispersion, as reported by Jun et al ${ }^{77,78}$, Yoon et al79, and Nair et a $\left.\right|^{80}$, but the mechanisms are not yet fully understood. There were no signs of significant fiber bundling or entanglement, based on the SEM images taken in different sections of the samples. The pore sizes, shown more clearly in the higher magnification images in Figure 10e-h, are in good agreement with the electrospun fiber diameters (Table 3).

a)

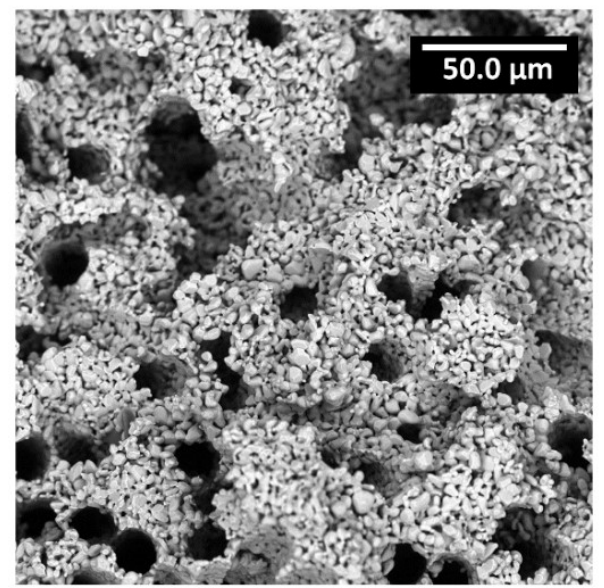

c)

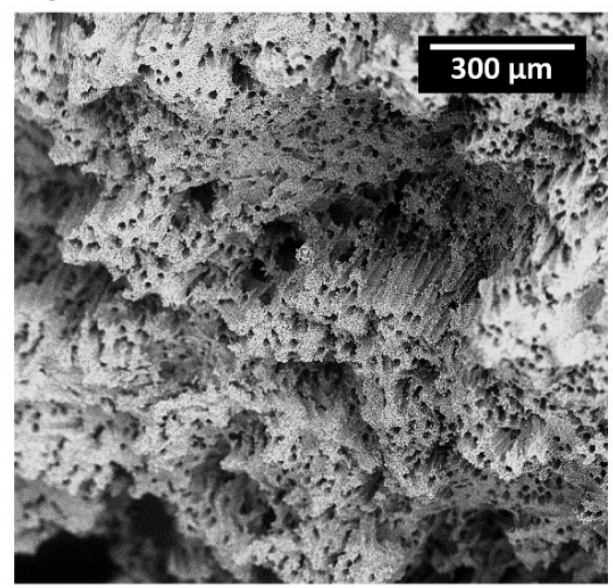

b)

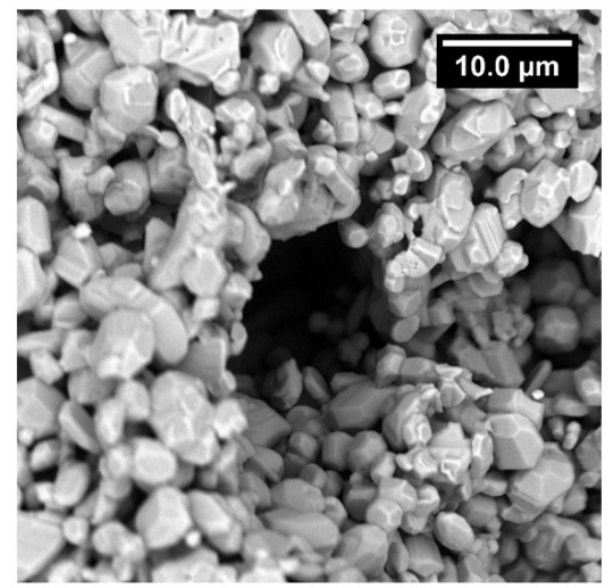

d)

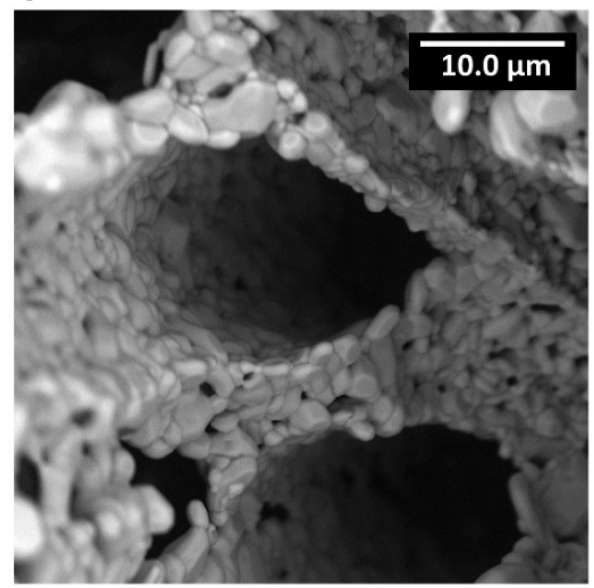

Figure 11: SEM micrographs of fresh-fractured surfaces perpendicular ( $a$ and $b$ ) and parallel ( $c$ and $d)$ to the to the fiber alignment to show the interparticle porosity in the struts and details of the alignment of the pores across the sintered porous $\mathrm{TiB}_{2}$ specimens. 
Apart from the macropores derived from the sacrificial fiber fillers, there is a second type of porosity among the $\mathrm{TiB}_{2}$ particles due to the partial sintering at $2000{ }^{\circ} \mathrm{C}$. This inter-particle porosity is in the range of 0.3-1 $\mu \mathrm{m}$ (Figure 11). The overall porosities (from the fibers and from the partial sintering) of these samples are summarized in Table 3, and range from 56 to $72 \%$ as the fiber diameter increases from 4 to $19 \mu \mathrm{m}$. Part of that porosity is based on the vol \% electrospun fibers in the mold (20\%) and the rest is from the partial sintering of the $\mathrm{TiB}_{2}$ particles. The combination of the processing route porosity and the partial sintering led to the creation of a multi-scale porosity that can be used for the tailoring of the thermal conductivity of the porous structures.

a)

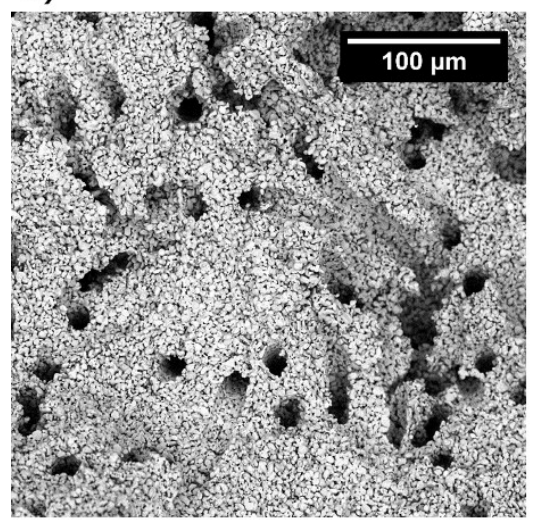

b)

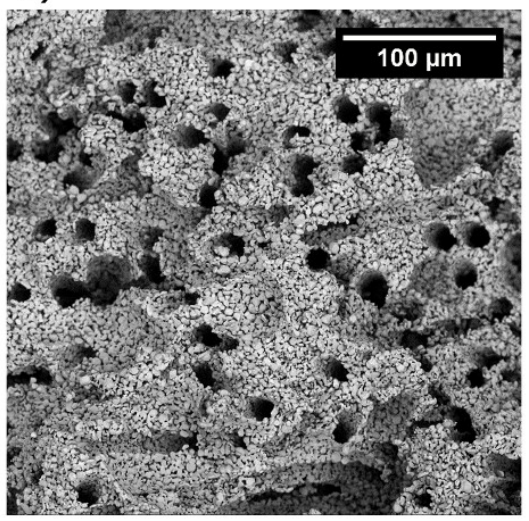

c)

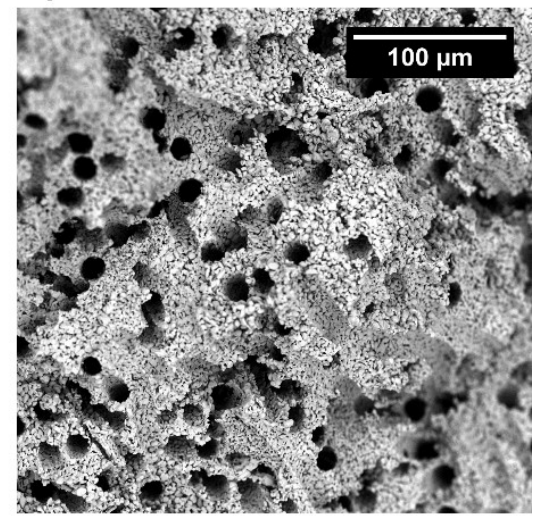

Figure 12: SEM micrographs of fresh-fractured surfaces perpendicular to fiber alignment of sintered TiB2 with fiber diameter of $17 \mu \mathrm{m}$ and different fiber amount: (A) 10, (B) 15, and (C) $20 \mathrm{vol} \%$.

Figure 12a-c shows SEM images of sintered samples prepared using a fiber diameter of $17 \mu \mathrm{m}$ and various amounts of PS fibers, 10, 15 and $20 \mathrm{vol} \%$ respect to the mold. The porosity increased from 58 to $72 \mathrm{vol} \%$ when the amount of fibers increased, as expected. These images show that, regardless of the different amount of fibers, there is an excellent dispersion of the pores across the samples with no need for additional surface modifiers, other than choosing 
ethanol as the solvent. As mentioned before, no significant evidence of bundling of fibers was observed in different sections of the samples. The pore size resembles with great accuracy of the fiber diameter, shown in Table 3. This is an indication of the ease of processing developed in this system.

\subsubsection{Advantages of Vacuum Infiltration Casting with Electrospun Fibers as Sacrificial Fillers}

Table 4: Pore size, porosity and inter-pore spacing for aligned pore structures.

\begin{tabular}{|c|c|c|c|c|c|}
\hline Techniques & $\begin{array}{l}\text { Pore Size } \\
\quad(\mu m)\end{array}$ & $\begin{array}{l}\text { Porosity } \\
\text { (vol \%) }\end{array}$ & $\begin{array}{c}\text { Average Pore } \\
\text { Spacing }+^{x} \\
(\mu \mathrm{m})\end{array}$ & $\begin{array}{c}\text { Percent } \\
\text { Standard } \\
\text { Deviation (\%) }\end{array}$ & $\begin{array}{c}\text { Spacing } \\
\text { Normalized by } \\
\text { Pore Size }\end{array}$ \\
\hline $\begin{array}{c}4(\mu \mathrm{m}) 20(\mathrm{vol} \%) \mathrm{PS} \\
\text { (this work) }\end{array}$ & 6 & 56.2 & 17.6 & 43.4 & 0.34 \\
\hline $\begin{array}{c}8(\mu m) 20(\text { vol \%) PS } \\
\text { (this work) }\end{array}$ & 8 & 48.8 & 19 & 40.7 & 0.42 \\
\hline $\begin{array}{c}17(\mu \mathrm{m}) 20(\mathrm{vol} \%) \text { PS } \\
\text { (this work) }\end{array}$ & 15 & 72.4 & 33.5 & 41.4 & 0.44 \\
\hline $\begin{array}{c}19(\mu \mathrm{m}) 20(\mathrm{vol} \%) \text { PS } \\
\text { (this work) }\end{array}$ & 21 & 71.5 & 43.8 & 32.3 & 0.48 \\
\hline BCP Templating 59 । & 0.02 & - & 0.0291 & 8.67 & 0.69 \\
\hline BCP Templating ${ }^{81 ~ ॥}$ & $3 \times 10^{-3}$ & - & 0.00484 & 38.9 & 0.6 \\
\hline Extrusion $^{66 ~ I I I}$ & 6 & 38 & 22 & 29.2 & 0.27 \\
\hline Freeze Casting $82 \mathrm{IV}$ & $4.5^{x x}$ & 42.2 & 13.1 & 29.8 & 0.34 \\
\hline
\end{tabular}

${ }^{+}$Distance from pore center to pore center

$x$ Pore spacing for this work was measured from Figure 5 e-h respectively.

$x x$ Measured with ImageJ following same procedure as for our materials.

' Measured from Figure 4 a, shown in Malenfant et al ${ }^{59}$.

"Measured from Figure 5, shown in Ciesla et al 81 .

III Measured from Figure 3 a, shown in Bae et al ${ }^{66}$.

IV Measured from Figure $3 \mathrm{c}$, shown in Araki et al ${ }^{82}$.

Table 4 shows a summary of the different pore sizes, overall porosities and inter-pore spacing obtained in this work, compared to other techniques reported in the literature to create aligned continuous pores in ceramic materials. That inter-pore spacing for our samples and for those techniques reported in the literature was quantified via an algorithm based on information 
gathered from the ImageJ software (see Appendix 1) to better compare the homogeneity of the pore distribution between different techniques. When the pore areas are calculated, their locations (centroids of the pores) can be stored and measured against the neighbor pores to gain insight into the inter-pore spacing. This was calculated for a pore by finding the closest neighbors surrounding that particular pore and repeating for every pore in the image. From this calculation, the average pore spacing and the corresponding standard deviation can be obtained (Table 4). The standard deviation is descriptive of the degree of order within the pore structure or homogeneity of the pore distribution. A low standard deviation will indicate pores that are consistently spaced or periodic. Inter-pore spacing was also normalized by the pore diameter, to allow for comparison with other techniques, regardless of the scale. The normalized pore spacing by the pore diameter, is analogous to pore density per area, where a value of 1 would mean that all the pores are touching side to side. This approach was applied to images of our samples and also to images published for the other techniques that produced aligned porosity. Table 3 indicates that for our samples, the pore size and the pore spacing increases as the fiber diameter increases, since there are fewer fibers of that size (same vol \% of fibers were used). Techniques that produce smaller pore sizes for a given porosity (like blockcopolymer techniques) exhibited a smaller pore inter-pore spacing ${ }^{59,81}$.

Compared to other techniques that create aligned porous structures, our technique has a pore spacing comparable to the other techniques that render similar pore sizes. For example, our samples exhibited a similar pore size, but lower pore spacing and higher overall porosity than the extrusion of dual porosity $\mathrm{LiCoO}_{2}$ by Bae et $\mathrm{al}^{66}$. The standard deviation indicated that our technique has a comparable pore periodicity or even-spacing, and the normalized pore spacing 
shows that our samples have more pores per area. The work of Araki et al ${ }^{82}$ on freeze casting (ice templating) presented a less ordered but still aligned porous structures. Our samples showed comparable number of pores per area, but more defined aligned continuous porous structures. Although these two previous techniques also produce samples in the macroporosity range, our method can create more uniform pores with very controlled pore sizes and shapes by using a relatively simple approach as extrusion and freeze casting. Block copolymers routes (for boron nitride presented by Malenfant et al $^{59}$ and for zirconium oxo phosphate by Ciesla et $\mathrm{al}^{81}$ ) showed a more highly ordered structure with a higher number of pores per area. By contrast, our processing technique can provide ordered porous materials in cast shapes, with comparably even pore spacing, at a lower cost and potential for scalability, even for a higher pore size.

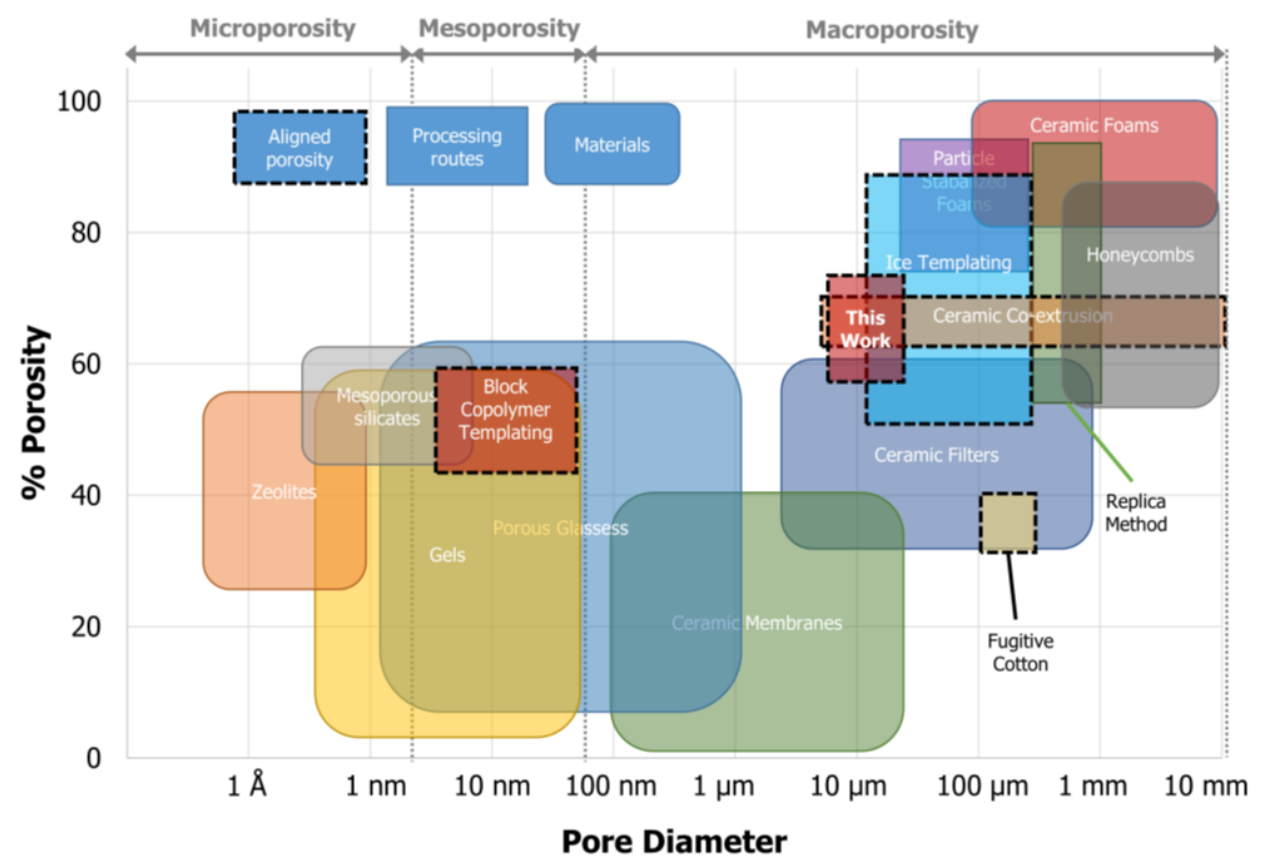

Figure 13: Comparative plot of porosity vs pore size for existing porous ceramic processing routes and the results of this work. 
The use of electrospun fibers as a sacrificial filler to create aligned continuous pores in a multiscale porous samples appears as an accurate and simple process. First, no additional surface modifiers, additives, or binders were needed to achieve the homogeneous pore distributions, other than the solvent choice and a well-known small addition of a ceramic commercial dispersant. Second, this process offers great versatility to tailor pore size and porosity by selecting the fiber diameter. Figure 13 shows a schematic with approximate bounds for porosity versus pore sizes for other processes used to prepare porous ceramic materials, including different pore morphologies, sizes and well-known porous materials, for the macro-, meso- and microporosity ranges. The bounds for the processing techniques that allow the creation of aligned continuous pores have been also depicted in the diagram. The results of the present work have been superimposed to compare with other routes. The use of electrospun PS fibers offers high control over porous structure, while maintaining the ease of processing, typical of sacrificial fillers processing routes. Our work enables the creation of not only smaller pores within the macropores regime, but also well-controlled and accurate alignment of high aspect ratio cylindrical pores. Compared to the processing routes that lead to continuously aligned pores, the combination of electrospun fibers and VIC process offer controlled pore morphologies and high porosities. 
a)

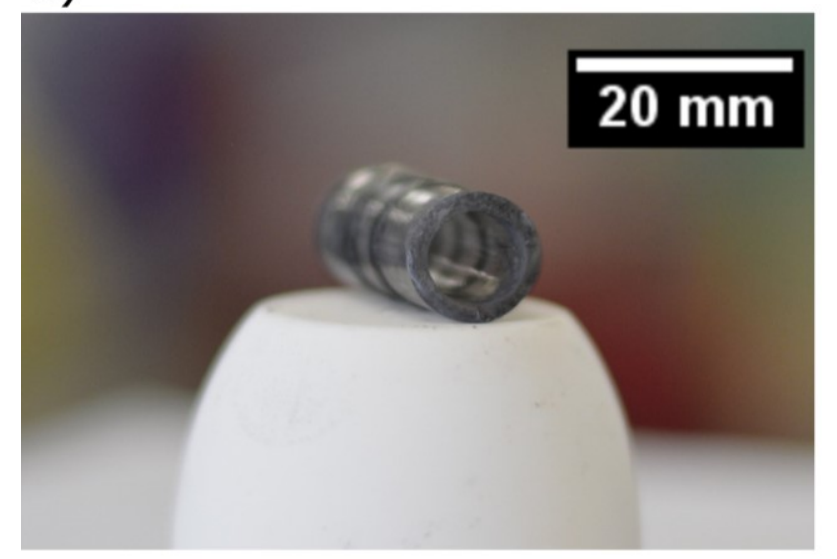

c) b)

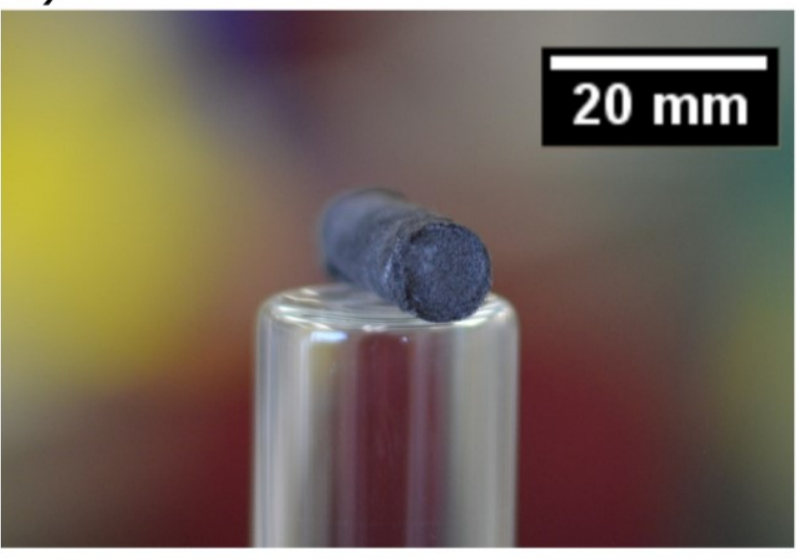

$20 \mathrm{~mm}$

Figure 14: Images of the: a) mold used for casting, b) standard $\mathrm{TiB}_{2}$ green body with aligned fibers, and c) a complex hollow green body with aligned fibers in the axial direction.

The VIC process described in this work using electrospun fibers also show potential to develop more complex shapes and different anisotropic pore designs while exhibiting a controlled porous structures across the piece. Figure 14 shows images taken with a digital camera of the mold and green bodies. The green bodies (Figure 14b and c) closely resembles the mold in dimension and shape demonstrating the cast-ability of the system. Figure 9c show a hollow cylinder with fibers aligned in the direction of the mold as a demonstration that more complex geometries can be attained. The alignment of the electrospun fibers and the use of the VIC process sets this sacrificial filler technique apart from the existing aligned pore processing 
routes, and its ease of processing and versatility make this approach suitable for a large range of ceramic materials.

\subsection{Conclusions}

Ceramic parts with aligned continuous pores have been produced by combining a vacuum infiltration casting approach with directionally aligned sacrificial fillers (PS fibers) produced by electrospinning. The fiber diameter and alignment were controlled during the electrospinning process to create PS fibers with diameters of 4, 8, 17 and $19 \mu \mathrm{m}$. $\mathrm{TiB}_{2}$ green bodies were produced by vacuum infiltration casting of the fiber-filler mold by a ceramic suspension. Aligned, continuous and evenly dispersed pores ranging from 5 to $20 \mu \mathrm{m}$ were derived from electrospun PS fibers, after pyrolysis and partial sintering. These samples exhibit a multi-scale porosity: pores from the sacrificial fillers and inter-particle pores from partial sintering. Samples with porosities values from 50 to $75 \mathrm{vol} \%$ were produced by either changing the fiber size or the fiber amount. The inter-pore spacing and pore density of the samples produced using these techniques exhibited similar values of inter-pore spacing, pore density and porosities to other macroporous techniques, but with pore morphologies definition similar to some mesostructured approaches. The simplicity of this approach represents a great potential to produce passive and microfluidics active cooling components with UHTCs for extreme applications, with tailored microstructures and geometries. 


\section{Chapter 2: Mechanical and Thermal Characterization of Continuous Aligned Porous $\mathrm{TiB}_{2}$}

\subsection{Introduction}

UHTC materials for active cooling components should be light weight, flexible, and are designed to be applied as non-loadbearing ${ }^{9}$. A combination of thermal conductivity, mechanical properties and oxidation behavior needs to be tailored for specific components, and an understanding of the processing-structure-properties relationship is essential for setting boundaries on the design space of porous UHTC materials ${ }^{83}$. Given a multi scale porous material from Chapter 1, the combined effect of the porous structure on thermal and mechanical behavior will be studied in controlled environments. The selected porous structure have pore sizes of 5, 10, 12 and $20 \mu \mathrm{m}$ produced following the route from Chapter1. 


\section{$\underline{2.1 .1}$ Heat Transfer in Porous Solids}

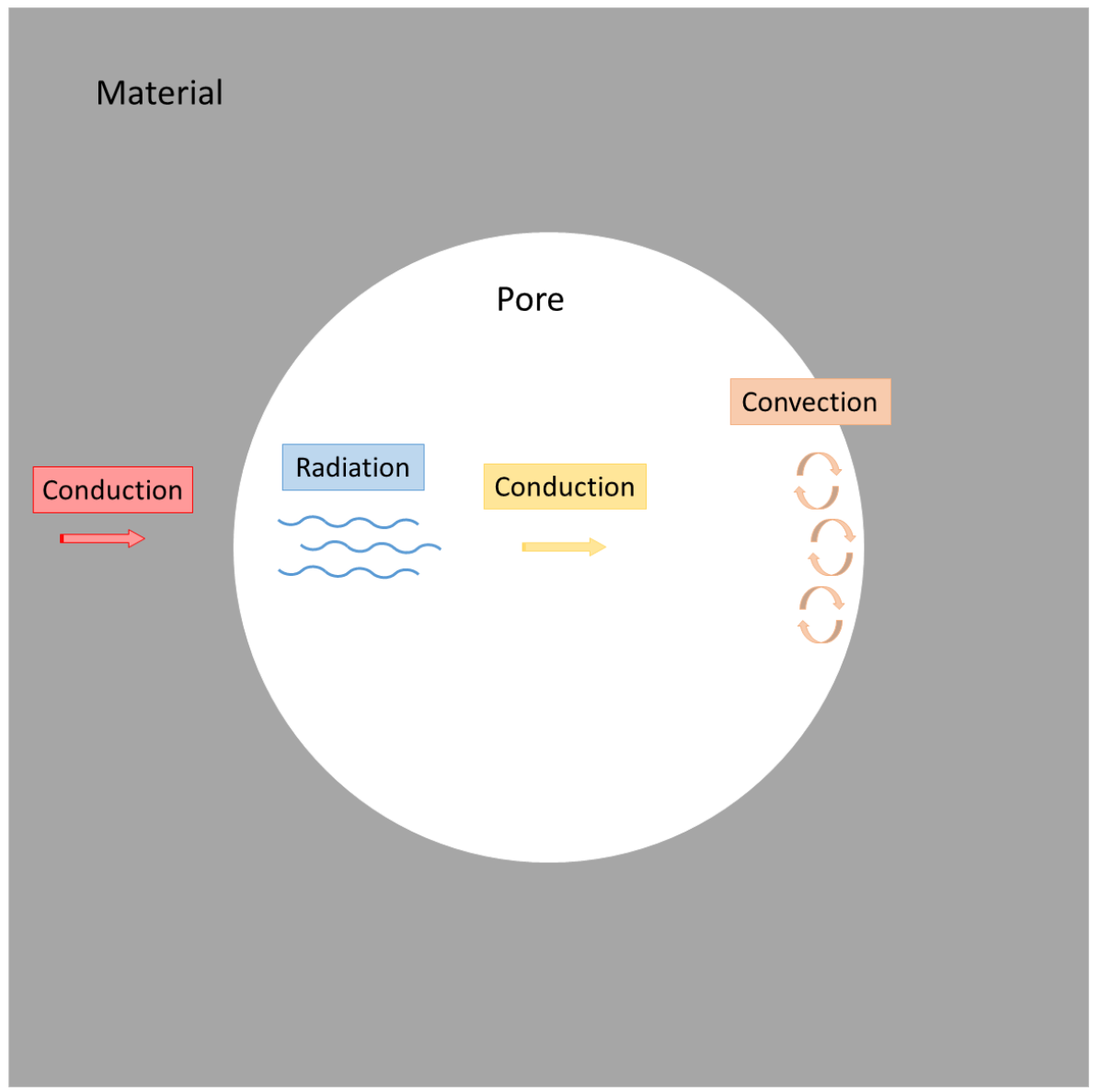

Figure 15: Paths for conduction in porous materials.

The effective conductivity $k_{e f f}$ of porous materials is generally described with a combination of 4 separate paths of heat transfer. These paths (shown in Figure 15) are the conductivity of the solid material $\left(k_{\text {solid }}\right)$, and gas phase $\left(k_{\text {gas }}\right)$ as well as the convection within the pores $\left(k_{\text {convection }}\right)$, and the radiation within pores $\left(k_{\text {radiation }}\right)$. These effects are often modeled separately and additively combined as in Equation $1^{41,84}$.

Equation 1: Thermal Conductivity Contributions.

$$
k_{\text {eff }}=k_{\text {solid }}+k_{\text {gas }}+k_{\text {convection }}+k_{\text {radiation }}
$$


Each contribution to conductivity can oscillate between being the dominant contribution to negligible depending on pore geometry and material solid properties. The conductivity of the solid and gas for insulating materials are both used to calculate a composite conductivity. A substantial number of methods exist to model effective conductivity for porous materials ${ }^{36}$.

Gas thermal conductivity is a function of temperature and will change depending on the gas. Our tests were carried out in argon to try and prevent oxidation. The temperature thermal conductivity of argon gas was fit by a least-squares analysis to a cubic function by Saxena et al over 350-2500 $K^{85}$, Equation 2.

Equation 2: Thermal Conductivity of Argon.

$$
k_{\text {gas }}=5.465+0.04729(T)-0.1111 * 10^{-4} T^{2}+0.1599 * 10^{-8} T^{3}
$$

Where $T$ is temperature in $K$. The conductivity of the gas has a larger contribution only when the conductivity of the solid material and gas are close in magnitude and/or porosity is above $90 \%{ }^{86}$, not the case with our materials.

Convection is heat transfer between the gas and material. Heat transfer by convection is not a function of the solid material properties. Gas flow and movement within a pore will increase convective transport, however, the intra-pore gas movement is not substantial enough to allow for convective heat transport for pores smaller than 4-5 $\mathrm{mm}^{41,84}$. Therefore, with our structure convection will be negligible.

Radiation is affected by both the pore geometry and the interaction of the material with infrared waves, substantiating a more complex analysis. There are several equations for 
estimating the radiation effect, rooted in the Stefan-Boltzmann law and Beer's Lambert $\operatorname{Law}^{87,88}$. The equation from Bhattacharijee et al. was used due to a limit placed by availability of literature values for the necessary inputs ${ }^{88}$. Equation 3 shows the form used by Bhattacharijee et al, where $\sigma$ is the Stefan-Boltzmann constant, $\varepsilon$ is the emissivity, $l$ is the pore length along the conducting path, and $\mathrm{T}$ is the mean temperature.

Equation 3: Radiation Contribution to Conductivity.

$$
k_{\text {radiation }}=4 \sigma \varepsilon l T^{3}
$$

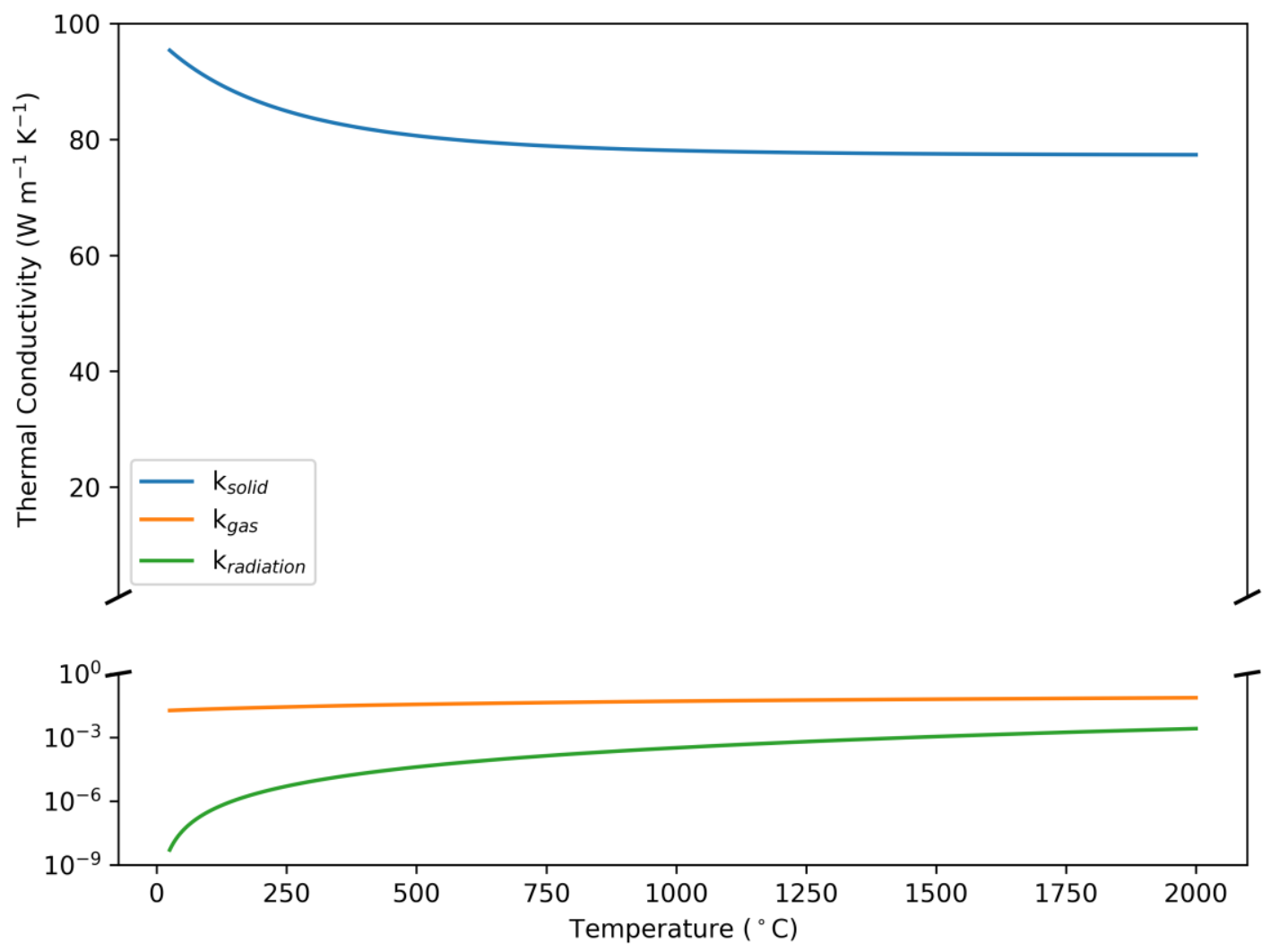

Figure 16: Thermal contributions plotted as functions on temperature, where $\boldsymbol{k}_{\text {solid }}$ represents dense TiB $\boldsymbol{B}_{2}, \boldsymbol{k}_{g a s}$ represents Argon, and $\boldsymbol{k}_{\text {radiation }}$ is based on $\mathrm{TiB}_{2}$ material properties. 
Figure 16 shows the thermal contribution from solid, gas, and radiation. The solid conductivity is based on an interpolation solved for by Munro, in a study of the thermal conductivity of $\mathrm{TiB}_{2}$ from $20-1200{ }^{\circ} \mathrm{C}^{14}$. The gas conductivity and the radiation contribution are both less than $0.1 \%$ than the solid conductivity, therefore they will not be considered when calculating the effective thermal conductivity for our material.

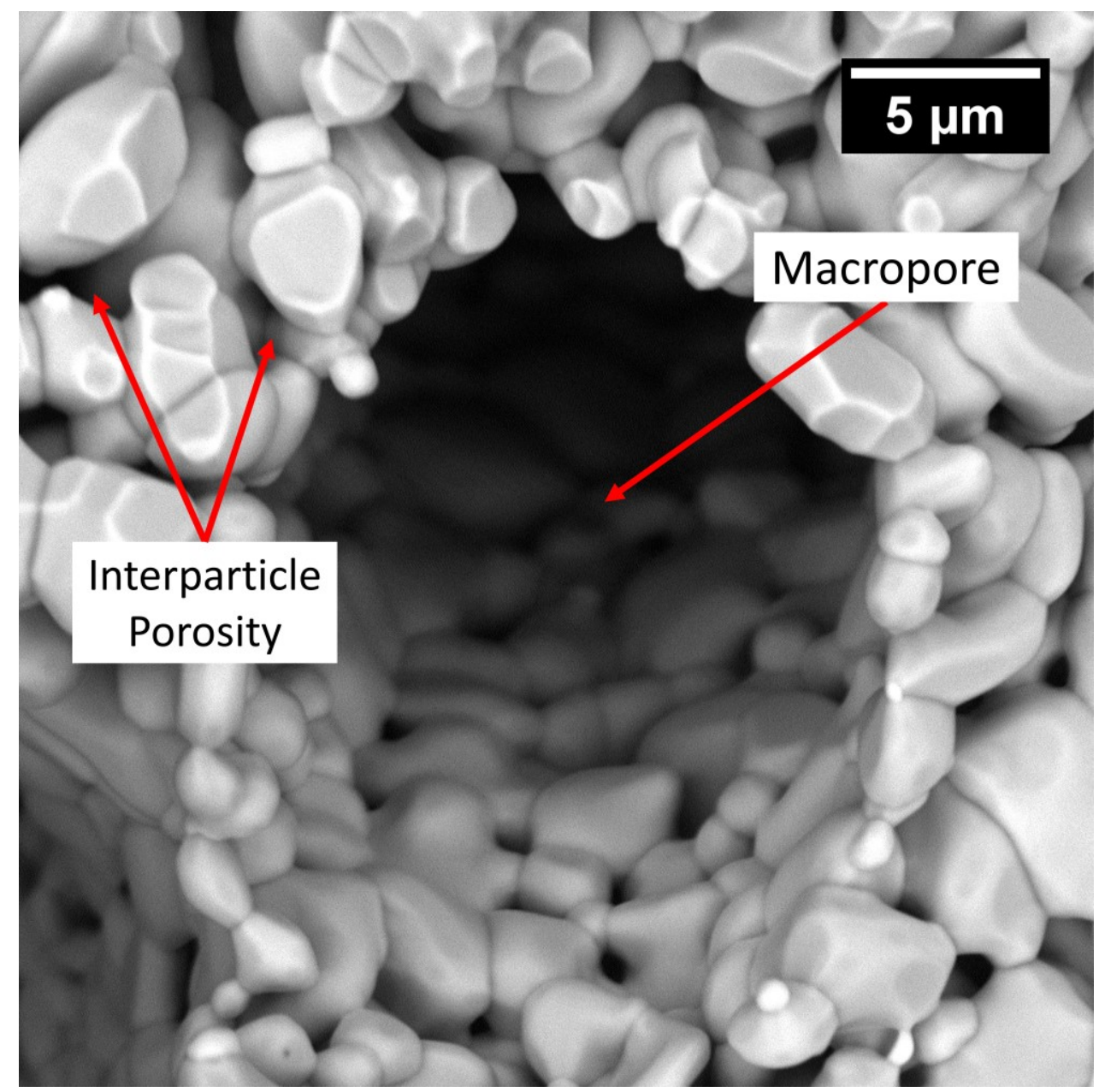

Figure 17: Example of a multiscale porosity with interparticle porosity and cylindrical pores from this work.

The two dominant forms of porosity in our microstructure are shown in Figure 17. To accurately connect structure to properties, a combination of analytical models that considers both the 
interparticle porosity from partial sintering and the macroporosity (cylindrical pores) from our sacrificial filler will be employed.

\subsubsection{Thermal Conductivity of Parallel Cylindrical Pores}

The cylindrical pores originate from the sacrificial filler route described in Chapter 1 . There are models for an anisotropic material composed of cylindrical aligned pores, but most of these are only concerned about heat flowing across the pore direction ${ }^{89,90}$. Li et al ${ }^{89}$ used a finite difference simulation to study the effect of effect of pore size and aspect ratio on thermal conductivity perpendicular to pore direction. They found decreasing conductivity with decreasing pore size, because of phonon scattering. The Rayleigh-Maxwell model for composite thermal conductivity, and often used for porous thermal conductivity, was modified by Hasselman et al to included resistance between the two phases. Hasselman et al derived a modified Rayleigh-Maxwell model for several geometries including conductivity perpendicular to the pore direction ${ }^{90}$. The effect of pore orientation on aligned lotus type porous copper has been modeled by Ogushi et $\mathrm{al}^{91}$ with good agreement with experimental data. Their model for conductivity parallel to the pore direction simplified to the rule of mixtures approximation, and predicted small changes in conductivity for misalignment of less than $10^{\circ}$ off of parallel alignment $^{91}$.

The use of the rule of mixtures for parallel cylindrical porous thermal conductivity makes sense when we consider heat flow as a probability of interference with a pore. Heat will be conducted parallel to pores and not be impeded by crossing multiple pores; there will simply be areas where there is material conduction, and areas of pore gas conduction. Because of this we have 
opted to use the parallel thermal conductivity approximation shown in Equation 4 to represent the contribution of the aligned pores and acts as the upper bound for conductivity ${ }^{20}$.

Equation 4: Parallel Thermal Conductivity

$$
k_{\text {eff }}=v_{\text {pore }} * k_{\text {solid }}
$$

Where $k_{\text {eff }}$ represents the effective thermal conductivity, $v_{\text {pore }}$ represents the volume fraction of pores and $k_{\text {solid }}$ represents the bulk solid material thermal conductivity.

\section{$\underline{2.1 .3}$ Interparticle Porosity Thermal Conductivity}

In addition to the cylindrical pores, the ceramic struts are composed of interparticle porosity, from partial sintering. Interparticle porosity has random orientation. The effect of a partially sintered structure was studied by Florez et al ${ }^{92}$, shown in Equation 5.

Equation 5: Partial Sintering Equation

$$
k_{\text {partially sintered }}=k_{\sigma} * \frac{2 *\left(1-v_{\text {pore }}\right)}{\pi * r}\left(\frac{0.56}{x}+\frac{8 * r *\left(1-\frac{r}{r+r_{m}}\right)}{\pi *\left(x+r \frac{x+r_{m}}{r+r_{m}}\right)^{2}}\right)^{-1}
$$

Where $k_{e f f}$ is the effective thermal conductivity, $v_{\text {pore }}$ is the porosity, $k_{\text {solid }}$ is the bulk conductivity (taken from Königshofer et al $^{93}$ and shown in Figure 16 as $k_{\text {solid }}$ ), $x$ is the radius of neck in plane of contact of two particles, $r$ is the particle radius, and $r_{m}$ is the radius of curvature of necked region between particles.

Equation 5 considers the geometry of the partially sintered particles within the porous body and does not account for radiation and grain boundary resistance where the disorientation of 
crystal lattices at grain boundaries can disrupt scatter phonons. In addition to the cylindrical pores and the interparticle porosity there is a small amount of additional random porosity form several sources: gas evolution during fiber burnout and areas where suspension did not fill during casting. In this case, for a sample with 50 vol \% porosity, we assume a constant 20 vol \% porosity from PS fibers, $30 \mathrm{vol} \%$ porosity in the struts from partial sintering, and the remainder being random porosity. This small portion of remaining porosity can be fit with a model by Rhee et $\mathrm{al}^{94}$, shown in Equation 6.

Equation 6: Aivazov-Domashnev Thermal Conductivity Fit

$$
k_{A-D \text { fit }}=k_{\text {solid }} * \frac{1-v_{\text {pore }}}{1+n * v_{\text {pore }}{ }^{2}}
$$

Where $k_{\text {solid }}$ is the conductivity of the bulk material, $v_{\text {pore }}$ is the volume faction of porosity and $\mathrm{n}$ is a fitting parameter. The fitting parameter $n$, which must be between 0 and 14 , was found empirically for a given material system and correlates to a specific material and pore structure below $74 \mathrm{vol} \%$ porosity. Rhee et al applied this equation to experimental data from a range of ceramics including polycrystalline graphite and alumina ${ }^{94}$. The small amount of random porosity modeled with this equation is well below $74 \%$.

\subsubsection{Mechanical Strength in Porous Solids}

As with thermal conductivity, there is a similarly complex relationship between porosity and mechanical strength. As porosity increases both mechanical strength and Young's modulus will decrease. In ceramics, failure happens when a crack forms in a pore or defect, and propagates far enough for failure to occur ${ }^{95}$. Cracks form where the local stress creates tensile forces great 
enough for material failure. There are several competing strategies for estimating strength of porous brittle materials. Some of the most popular are 1) Minimum Solid Area (MSA) $\left.{ }^{96}, 2\right)$ pore stress concentration factor theory ${ }^{97}$, or the 3) Gibson Ashby cellular solid models ${ }^{98}$.

\subsubsection{Minimum Solid Area Method}

a)

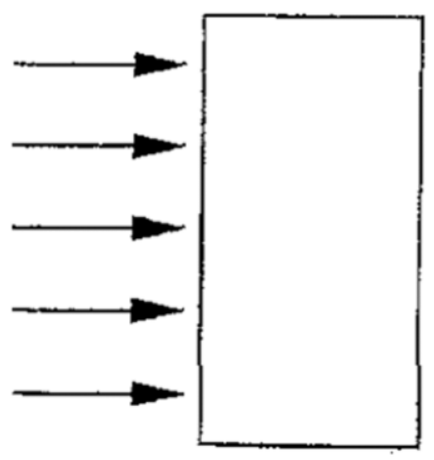

c)

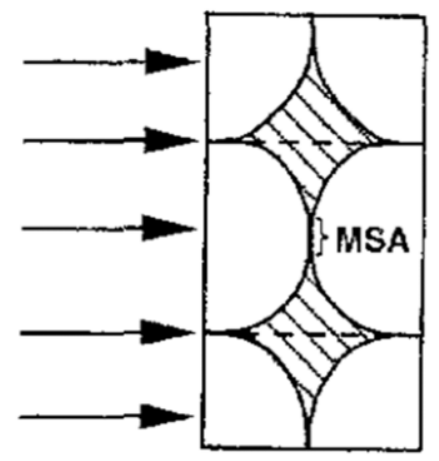

b)

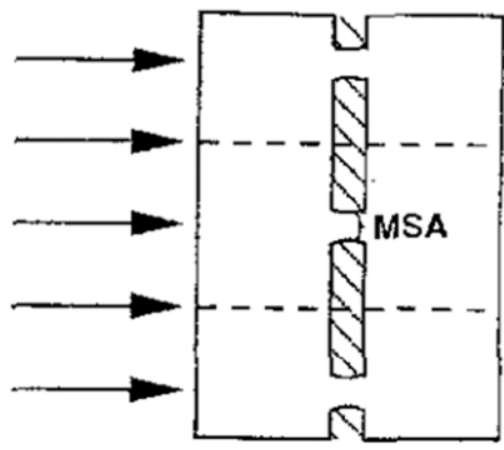

d)

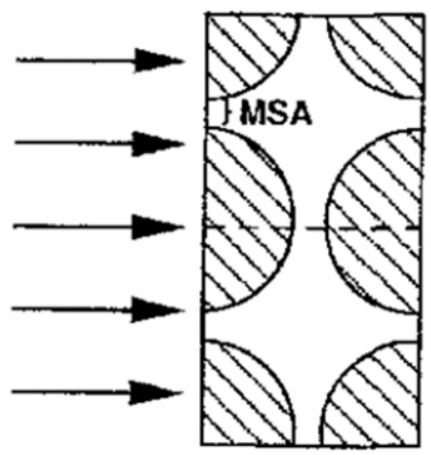

Figure 18: Minimum Solid Area Scheme A) a solid material where the load will be uniformly distributed, B) mostly solid are with a region of porosity reducing the area of material in that section, $C$ ) spheres packed where the contact points represent the minimum solid area and D) a cellular solid where the struts represent the minimum solid area reproduced from Rice "reuse with permission"

The MSA approach suggests that strength can be estimated by the amount of solid area where a force is applied and the properties of the solid material. This model assumes a uniform and 
even pore structure. Figure 17 shows some basic configurations for porous structures. Reynaud et al $^{99}$ studied the mechanical properties a random porous material of SiC from 3-37 vol \% porosity and showed their conformation to the MSA theory. Recent work by Pabst et al ${ }^{100}$ has attempted to refute the MSA model, stating that it is only valid for highly anisotropic materials and showing that MSA is incorrect for the simplest cases of partially sintered porous structures. The MSA approach is a simple method for modeling porosity, but due to our multiscale porosity, both isotropic and anisotropic in form, this would not be appropriate to describe our structure property relationship.

\subsubsection{Stress Concentration Factor Method}

a)

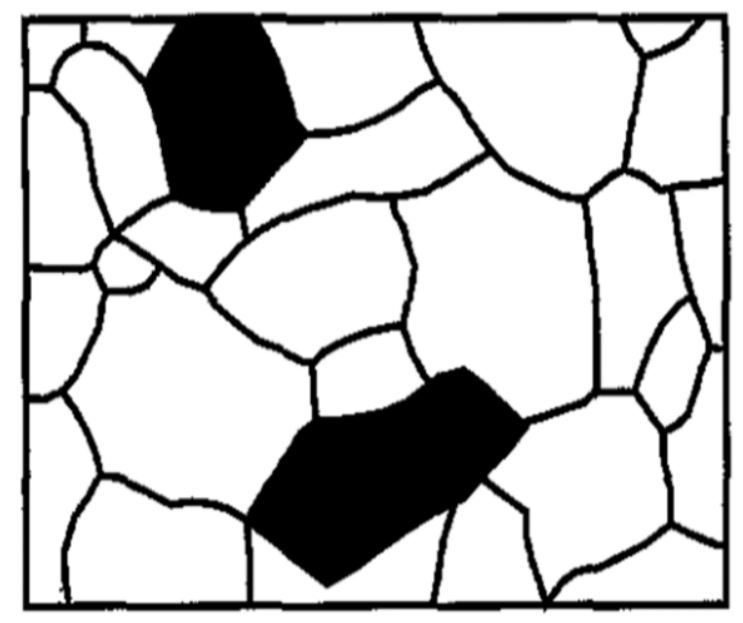

b)

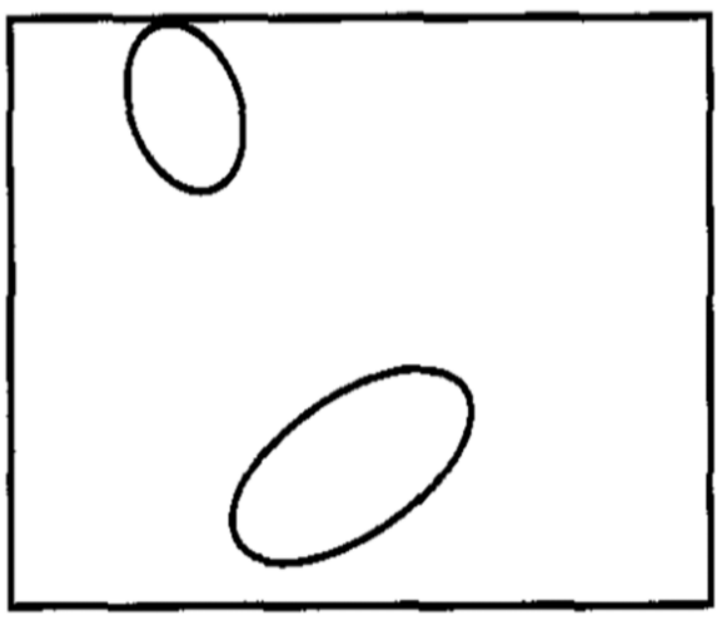

Figure 19: Stress Concentration Factor Scheme a) real microstructure and b) ellipse representation for model estimate extracted from Boccaccini et al "reuse with permission"97.

The stress concentration factor method assumes that failure will occur at the highest stress concentration or strength limiting feature of the structure. In ceramic materials with porosity in the $20-40 \%$ range mechanical strength has a strong correlation with stress concentration 
factor ${ }^{97,101,102}$. The stress concentration model described by Boccaccini et al ${ }^{97}$ includes parameter to describe the dimensions and orientation of the ellipse that represents the stress concentration feature pore to determine properties. Cox et al applied the model to an anisotropic porous $\mathrm{Zr}$-based bulk metallic based glass foam. The material was studied over porosity ranging from 39-54 $\mathrm{vol} \%$ and pore orientation from 0 to $90 \mathrm{vol} \%$ and found reasonable agreement with the model. Boccaccini et al state that pores parallel to the stress direction will not cause stress concertation ${ }^{97,103,104}$. Our cylindrical pores are mostly parallel to the stress direction and the interparticle pores will be randomly oriented, therefore this model will not properly represent our system.

\subsubsection{Gibson Ashby Cellular Solid Approach}

Gibson and Ashby present a more comprehensive model where they divide the materials into unit cells and discuss the forms of loading within those cells. The Gibson Ashby model considers the expected loading within a potential structure and uses a derivation of the stress fields to pinpoint failure criterion. There are three general pore structures discussed by Gibson and Ashby: closed cell, open cell, and honeycomb shown in Figure 20.

a)

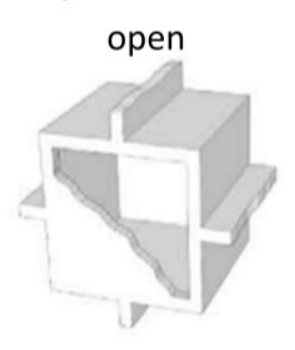

b)

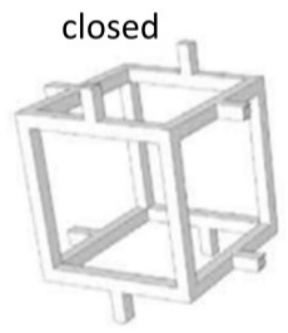

c) honeycomb

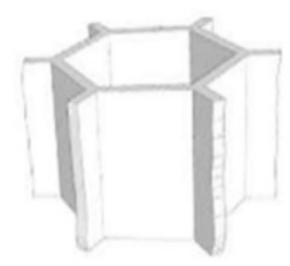

Figure 20: Gibson and Ashby cellular solid cell geometries a) closed cell, b) open cell and c) honeycomb extracted from Seuba et al "fair use"24. 
The Gibson Ashby model has separate equations for elastic plastic and brittle materials, referring to the bulk material behavior. The closed cell model refers to closed pores filled with a compressible gas, Equation 7.

\section{Equation 7: Closed Cell}

$$
\sigma=\sigma_{P}\left(C_{6}\left(\phi * \frac{P^{*}}{P_{0}}\right)+C_{6}^{\prime \prime} *(1-\phi) * \frac{P^{*}}{P_{0}}\right)
$$

Where $\sigma$ is the effective compressive strength, $\sigma_{P}$ is the modulus of rupture of the solid material within the walls, $C_{6}$ and $C_{6}^{\prime \prime}$ are geometrically determined constants, $\phi$ represents the volume fraction of porosity in the walls, $P^{*}$ is the density of the porous structure and $P_{0}$ is the density of the solid material ${ }^{24}$.

Open cell is similar in shape to closed cell except that the pore diameters are larger than the cell length and the pores overlap cells, Equation 8.

\section{Equation 8: Open Cell}

$$
\sigma=\sigma_{P} * C_{4}\left(\frac{P^{*}}{P_{0}}\right)^{3 / 2}
$$

Where all variables are the same as in Equation 7 and $C_{4}$ is a experimentally determined constant.

Honey comb represents an anisotropic structure composed of hexagonal cells ranging in length from continuous through the materials to short stacked cells, Equation 9. 
Equation 9: Honey Comb

$$
\sigma=6 * E_{s}\left(\frac{P^{*}}{P_{0}}\right)^{3}
$$

Where all variables are the same as in Equation 7 and $E_{S}$ is the Young's modulus of the dense material.

Closed cell solids are typically polymer foams where the cell walls can be thin with no flaws such that a gas could be trapped inside the pores. Ceramics produced with direct foaming are open cellular materials. Materials with ice templated structures are normally approximated as honey comb even though they have cylindrical pores. The variables for fitting the material in this study are presented in the work of Seuba et al ${ }^{24} . C_{6}, C_{6}$, and $C_{4}$ are experimentally determined fit variables for brittle materials. 



Figure 21: Gibson Ashby Models plotted with normalized compressive strength highlighting different structures A) replica, B) foaming, C) pore former and D) ice templating. The title shows the cell structures in order of closed cell, open cell and honey comb reproduced from Seuba et al "fair use"24.

Seuba et al produced research on pore diameter and wall thickness vs mechanical properties in freeze cast ceramics, and a review of porous ceramic mechanical behavior compared with the Gibson Ashby model. Figure 21 from Seuba et $\mathrm{al}^{24}$ shows several forms of cellular ceramics, open cellular from replica, foaming and pore former and honeycomb from ice-templating, materials plotted against the Gibson Ashby model. The replica methods fall slightly below the open cell and honey comb model because of burnout defects. Ice templated ceramics follow the honey comb model. Sample prepared from replica, foaming and pore formers fall between open and closed cell. 
Table 5: Gibson Ashby cellular solids model parameters for brittle materials.

\begin{tabular}{|c|l|l|}
\hline \multicolumn{2}{|l|}{ Closed Cell } & Solid Material Modulus of Rupture \\
\hline$\sigma_{P}=$ & $1 \mathrm{MPa}$ & Fit Constant for Ceramic Brittle Behavior \\
\hline$C_{6}=$ & 0.65 & Solid Fraction in the Cell Edges \\
\hline$\phi=$ & $30 \%$ & Solid Material Density \\
\hline$P^{*}=$ & $4.52 \mathrm{~g} \mathrm{~cm}^{-3}$ & Apparent Material Density \\
\hline$P_{0}=$ & Independent variable & Fit Constant for Ceramic Brittle Behavior \\
\hline$C_{6}^{\prime \prime}=$ & 1 & \multicolumn{1}{|l|}{} \\
\hline Open Cell (out of plane) & Solid Material Modulus of Rupture \\
\hline$\sigma_{P}=$ & $1 \mathrm{MPa}$ & Fit Constant for Ceramic Brittle Behavior \\
\hline$C_{4}=$ & 0.2 & Solid Material Density \\
\hline$P^{*}=$ & $4.52 \mathrm{~g} \mathrm{~cm}^{-3}$ & Porosity \\
\hline$P_{0}=$ & Independent variable & Solid Material Young's Modulus \\
\hline Honey Comb & \multicolumn{2}{|l}{} \\
\hline$E_{S}=$ & $500 \mathrm{GPa}$ & Porosity \\
\hline$P_{0}=$ & Independent variable
\end{tabular}

To accurately interpret the mechanical behavior, it is important to determine how the loading, deformation and pore structure will contribute to failure. The Gibson Ashby model has been shown in literature as an accurate analytic model to describe mechanical behavior in porous ceramics, and it will be used in this work to understand the mechanical response of the multiscale aligned porous samples.

In this chapter, we will measure the thermal and mechanical response of structures following the processing route outlined in Chapter 1, with materials of pore sizes of 5, 10, 12 and $20 \mu \mathrm{m}$. The structure-property relationship for the multi-scale porous materials will illuminated with a combination analytical models to describe the roles of interparticle porosity and aligned macroporosity in thermal conductivity and mechanical properties. These measurements are 
taken and compared to models with the goal of forming a more rigid bounds for the design space of porous UHTCs.

\subsection{Materials and Methods:}

\subsubsection{Samples}

Fibers with sizes 5, 10, 12 and $20 \mu \mathrm{m}$ were used to produce porous samples as described in Chapter 1 . Slip cast samples were produced to create a material that had the interparticle porosity and therefore the properties of the struts. Slip cast samples were made with the 30 vol \% suspension used in Chapter 1 , and slip cast in a cylindrical mold on top plaster of paris. The slip cast samples were $10 \mathrm{~mm}$ in diameter, while the aligned porous samples were $8 \mathrm{~mm}$ in diameter due to differences between the casting apparatus and molds. All porous and slip cast samples were sintered at $2000^{\circ} \mathrm{C}$ (following the same cycle described in Chapter 1 ).

Thermal conductivity samples were cut into $2 \mathrm{~mm}$ thick discs whether porous or slip cast. The $20 \mu \mathrm{m}$ sample was also prepared for $\mathrm{x}$-ray computed tomography characterization prior to diffusivity testing. Samples were cut with a low speed saw (Buhueler Isomet 1000), and faces made parallel with sand paper (3M) for mechanical testing. Porous samples for mechanical testing had a diameter and height of $8 \mathrm{~mm}$, and slip cast samples were $10 \mathrm{~mm}$ in height and diameter to maintain a constant $1 / 1$ ratio between samples.

\subsubsection{Porosity}

Porosity was measured for each sample using the Archimedes method, similar to the thermal conductivity samples. Cylindrical pore size was taken from previous trends in processing Chapter 1, and confirmed with image analysis using Image (version: 1.51j8, Wayne Rasband, 
National Institute of Health, USA) in some samples from a Table Top SEM (Phenom Pro, Model 800-07333, Phenom World, USA). Table 6 and Table 7 show the porosity and pore size values for samples used for further characterization.

Table 6: Thermal conductivity sample properties.

\begin{tabular}{|l|l|l|l|l|l|}
\hline Pore Size $(\boldsymbol{\mu m})$ & 10 & 10 & 12 & 12 & 20 \\
\hline Porosity $(\boldsymbol{v o l} \%)$ & 59 & 61 & 56 & 74 & 77 \\
\hline
\end{tabular}

Table 7: Mechanical testing sample properties.

\begin{tabular}{|l|c|c|c|c|c|c|c|c|c|}
\hline Pore Size $(\boldsymbol{\mu m})$ & 5 & 10 & 10 & 20 & 20 & 20 & Slip Cast & Slip Cast & Slip Cast \\
\hline Porosity (vol \%) & 65 & 60 & 59 & 71 & 71 & 73 & 30 & 30 & 29 \\
\hline
\end{tabular}

\subsubsection{X-ray Computed Tomography (X-ray CT)}

X-ray CT was measured at the Cornell Biotechnology Resource Center Imaging Facility (Cornell

University, Ithaca, New York) with a Zeiss/Xradia 520 X-ray Microscope. Reconstruction was done using MITK software (German Cancer Research Center Division of Medical Image Computing, Heidelberg, Germany.) Slices were converted to images for analysis using python.

\subsubsection{Thermal Measurements}

Thermal diffusivity was measured by Oakridge Nation Lab, using a Netzsch LFA 457 (Netzsch, Selb, Germany) Microflash diffusivity system. Measurement and signal processing followed ASTM E1461-13 105 . The Netzsch LFA 457 was outfitted with a quartz tube furnace, SiC sample holders and run under ultra-high purity argon. $\mathrm{O}_{2}$ concentration was estimated to be $100 \mathrm{ppm}$. Thermal conductivity was calculated from diffusivity data following ${ }^{105}$ where $\alpha$ is the diffusivity at a given temperature, $C_{p}$ is the heat capacity at a given temperature calculated according to 
the formula provided by $\mathrm{NIST}^{14}$, and $\rho$ is the sample density. $C_{p}$ was calculated following the Shomate Equation fit by Munro et al ${ }^{14}$ from RT to $2000^{\circ} \mathrm{C}$.

Equation 10: Thermal Diffusivity Thermal Conductivity Relation

$$
k=\alpha * C_{p} * \rho
$$

\subsubsection{Mechanical Testing}

Mechanical specimens were measured using an Instron 5960 Series Dual Column Table Frame (Instron, Norwood, MA). Porous samples were loaded parallel to aligned porosity. A Crosshead speed of $0.1 \mathrm{~mm} / \mathrm{min}$ was used. Strain was calculated from crosshead displacement.

\subsection{Results and Discussion:}

\subsection{1 $\mathrm{X}$-ray $\mathrm{CT}$}

Figure 22 , show a 3D reconstruction of a representative microstructure with $20 \mu m$ pores in the parallel direction. As in the SEM images, the pores seem well dispersed and mostly aligned. The tomography shows some of the defect areas that resulted from fiber burnout or air bubbles during casting. The resolution used for Figure 22 is $4 \mu \mathrm{m}$ such that the pores and large defects can be resolved but interparticle porosity cannot be seen. 


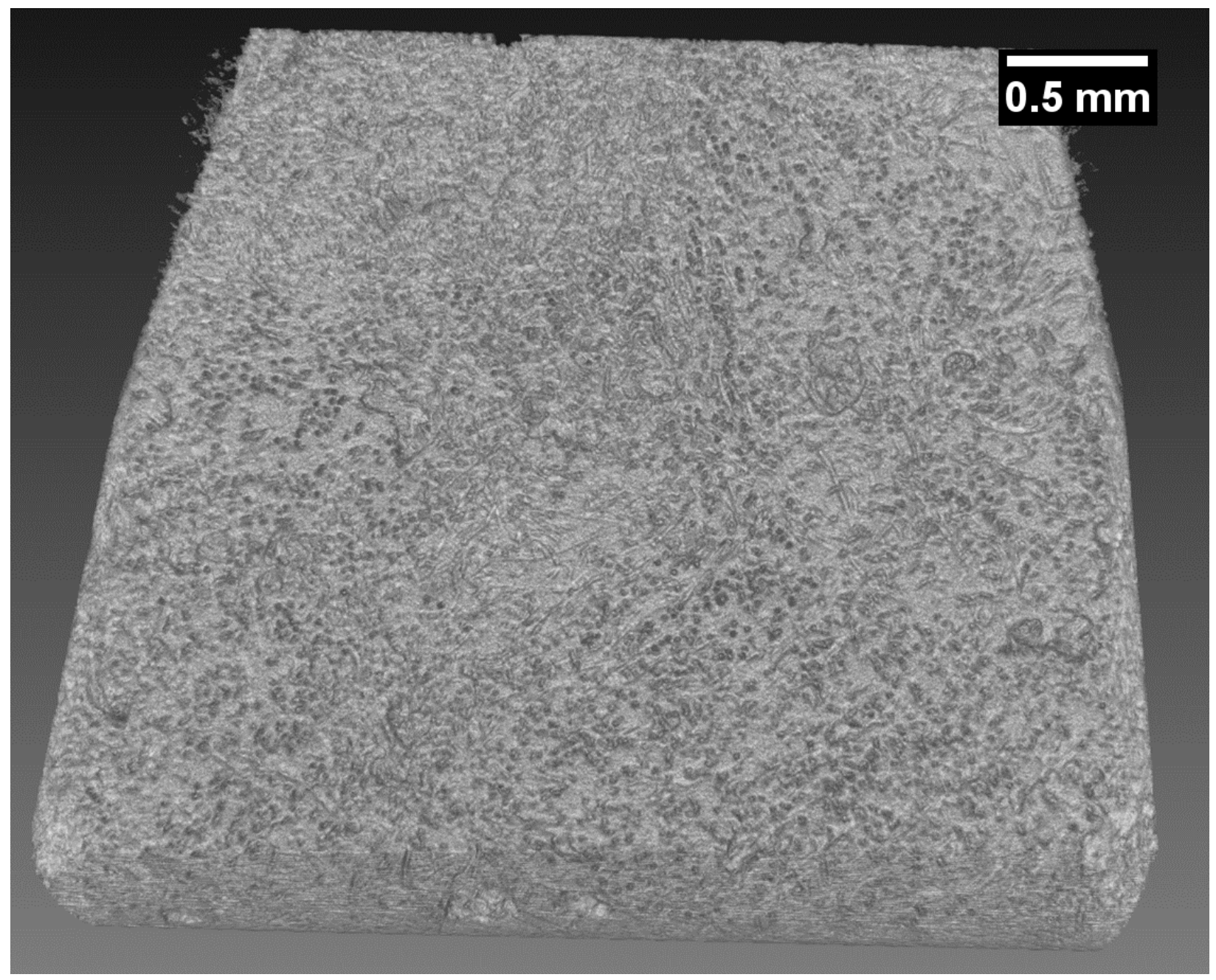

Figure 22: X-ray computed tomography section of $20 \mu \mathrm{m}$ sample at $4 \mu \mathrm{m}$ voxel size to show macropore structure without partially sintered pores.

X-ray CT data was used to visualize and understand the 3D microstructure. Porous UHTC materials of $\mathrm{ZrB}_{2}$ and $\mathrm{TiB}_{2}$ made from partial stabilized foams, ice-templating, and reticulated foam templates have been reconstructed using X-ray CT and used as inputs for thermal and mechanical models ${ }^{22}$. Partially sintered perovskite $\mathrm{La}_{0.6} \mathrm{Sr}_{0.4} \mathrm{Co}_{0.2} \mathrm{Fe}_{0.8} \mathrm{O}_{3-\delta}$ (LSOF), a cathode material, with varying porosity was reconstructed from FIB-SEM data and input into a mechanical model to connect the processing-structure-properties relationship ${ }^{106}$. X-ray CT has been used to reconstruct $39 \%$ porous alumina after compression testing to understand the 
failure ${ }^{107}$. Recent improvements in the last two decades in data collection and processing have allowed for $\mathrm{x}$-ray $\mathrm{CT}$ to become an increasingly common technique for understanding materials structure-processing-properties relationships.

The $\mathrm{x}$-ray CT was not used as an input into a model for mechanical or thermal properties. To sufficiently take advantage of X-ray CT data to study the effects of pore size and porosity of our material, data would need to be gathered for all pore sizes and porosities. The data is currently serving as visualization and insight into the 3D microstructure not fully realized through SEM. 


\subsubsection{Thermal Conductivity}

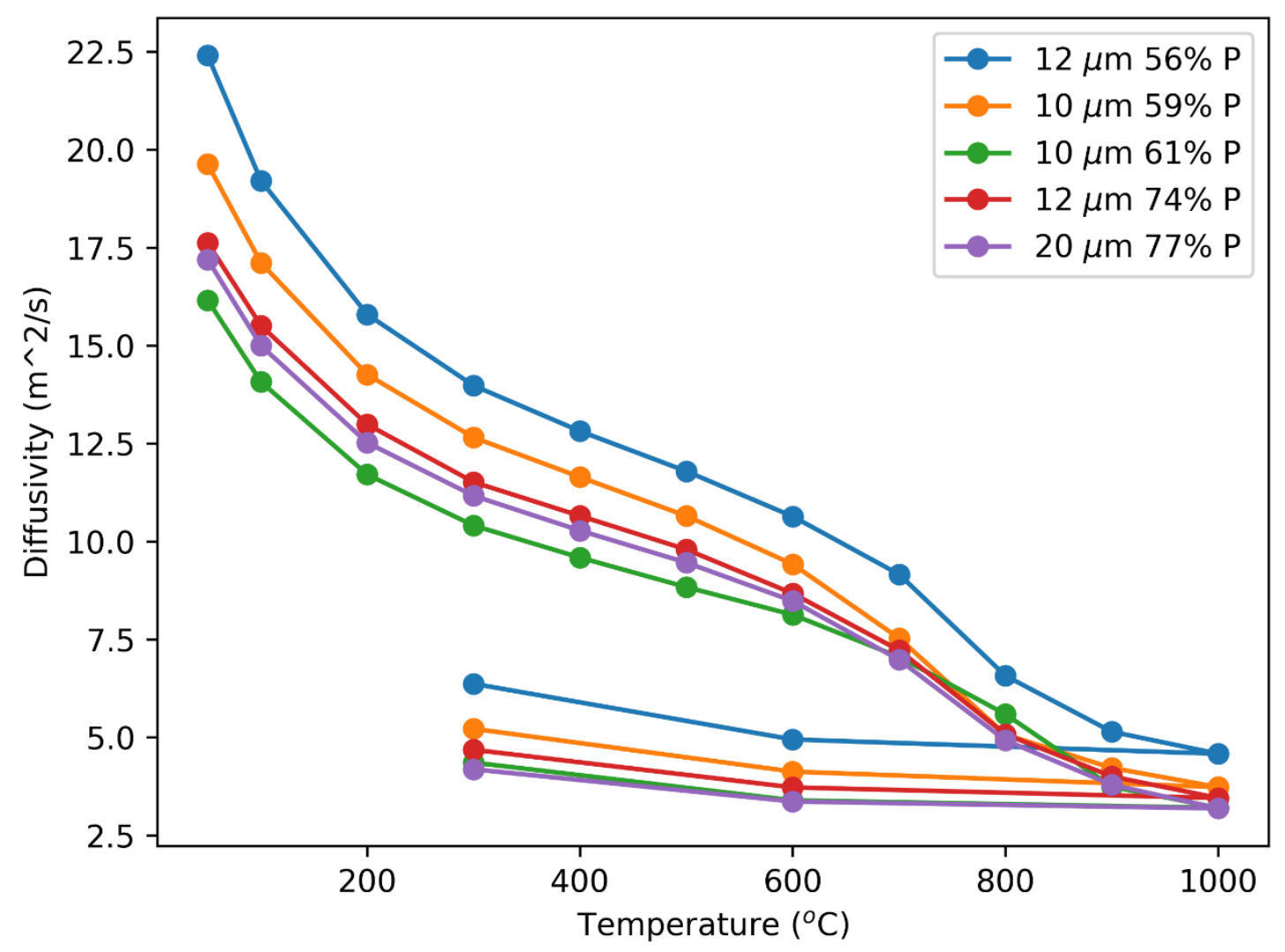

Figure 23: Thermal Diffusivity as a function of temperature for porous samples with 10, 12 and $20 \mu \mathrm{m}$ sized pores, measured at Oak Ridge National Lab.

Figure 23 shows the curves of measured diffusivity, where diffusivity decreases as porosity increases. The curves all follow a similar trend of decreasing diffusivity with increasing temperature, a slope change initiating around $600^{\circ} \mathrm{C}$ and a severe hysteresis upon cooling. 


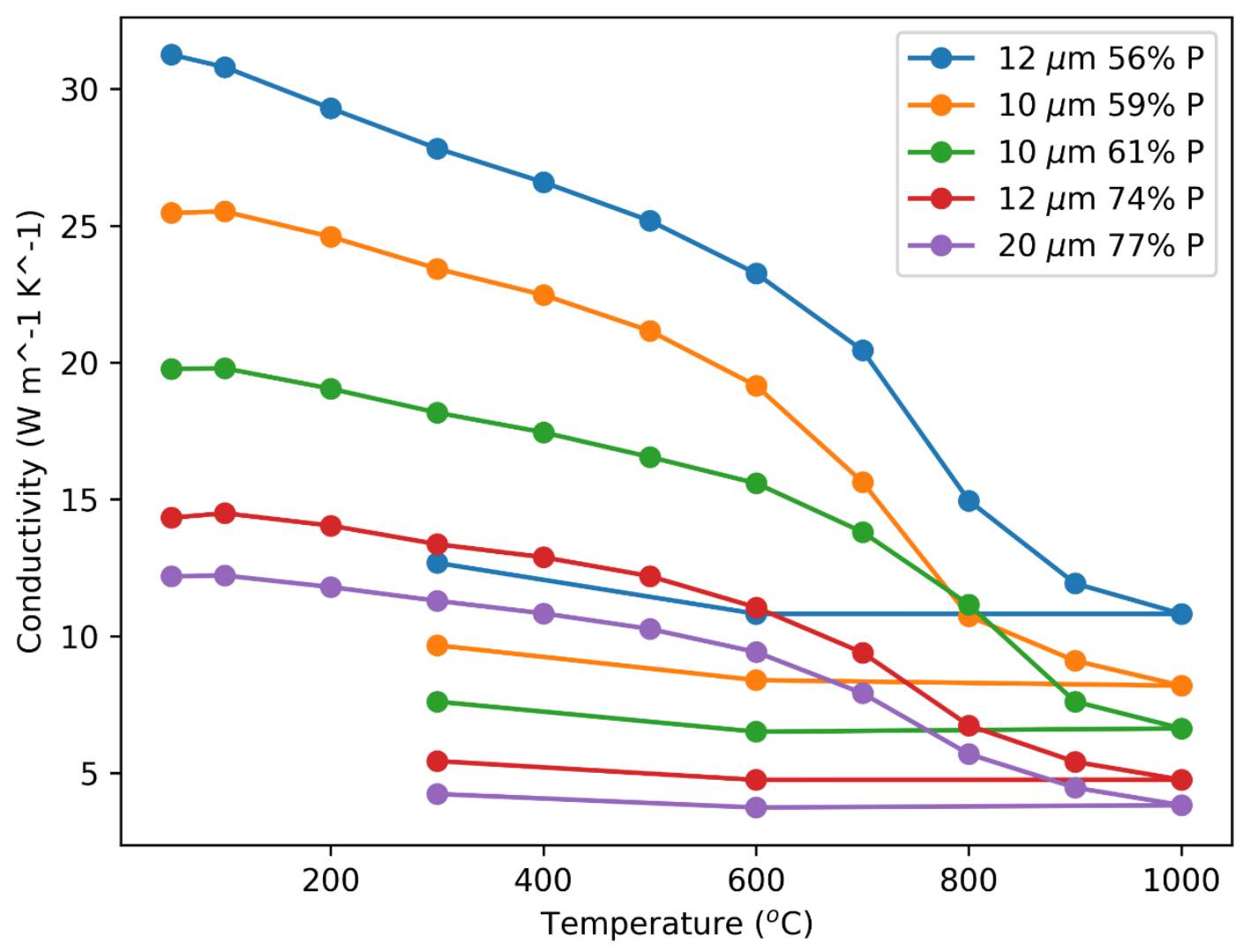

Figure 24: Thermal Conductivity as a function of temperature for porous samples with 10, 12 and $20 \mu m$ sized pores, measured at Oak Ridge National Lab.

Figure 24 shows the thermal conductivity plotted as a function of temperature. The samples show decreasing thermal conductivity with increasing porosity across the samples, as expected. Initially we see that conductivity decreases with increasing porosity. We do not see a strong association of pore diameter with conductivity, indicating that the conductivity is independent of variations in pore sizes for the distribution we studied when heat flow is parallel to the pores, but is strongly dependent on overall porosity.

Each of the curves can be separated into three regions. First we see a minute increase in thermal conductivity as the temperature rises from RT until $100^{\circ} \mathrm{C}$. A similar phenomena has 
been seen in the resistivity vs temperature curves of other measured UHTCs ${ }^{93,108-111 .}$ Königshofer et al ${ }^{93}$, observed a similar increase in electrical and thermal conductivity from RT to $100{ }^{\circ} \mathrm{C}$ in dense hot-pressed $\mathrm{TiB}_{2}$. Justin et al ${ }^{108}$ saw and initial rise in thermal conductivity for dense $\mathrm{HfB}_{2} / \mathrm{SiC} / \mathrm{TaSi}_{2}$ from $\mathrm{RT}-300^{\circ} \mathrm{C}$. This initial rise, only seen in the conductivity curve is a result of a rapid increase in heat capacity happening over the same temperature range ${ }^{14}$. However, several other papers do not have this initial increase in conductivity with temperature $\mathrm{e}^{39,110,112,113}$.

Second, from $100-200{ }^{\circ} \mathrm{C}$ to $600-700^{\circ} \mathrm{C}$ there is a decrease in conductivity with temperature, typical for lattice thermal conductivity, where the phonon mean free path is becoming increasingly smaller as the material approaches the Debye temperature. Additionally, phonon scattering off grain boundaries and defects can contribute to further lowering thermal conductivity. The thermal conductivity for dense $\mathrm{TiB}_{2}$ typically decreases from 90 to 78 $\mathrm{Wm}^{-1} \mathrm{~K}^{-1}$ over the temperature range $100-600{ }^{\circ} \mathrm{C}^{14,93}$. Tallon et a ${ }^{22}$ measured the thermal conductivity of several porous UHTC materials and reported ice-templated $\mathrm{ZrB}_{2}$ at $51 \mathrm{vol} \%$ porosity and $24 \mathrm{Wm}^{-1} \mathrm{~K}^{-1}$ and $\mathrm{ZrB}_{2}$ foam at $68 \mathrm{vol} \%$ porosity and $24 \mathrm{Wm}^{-1} \mathrm{~K}^{-1}$. $\mathrm{TiB}_{2}$ produced with several different techniques show a range of conductivities: ice-templating at 56 vol \% porosity with macropore pore sizes from 30 to $100 \mu \mathrm{m}$ and similar interparticle porosity shows a RT thermal conductivity of $23.4 \mathrm{~W} \mathrm{~m}^{-1} \mathrm{~K}^{-1}$, foaming resulted in a porosity of 84 vol $\%, 80-200 \mu m$ pores and a conductivity of $2.88 \mathrm{~W} \mathrm{~m}^{-1} \mathrm{~K}^{-1}$ at $\mathrm{RT}$ and $\mathrm{TiB}_{2}$ made through a replica technique with $0.5 \mu \mathrm{m}$ pores and $93 \%$ has a conductivity of $1.15 \mathrm{~W} \mathrm{~m}^{-1} \mathrm{~K}^{-1}$ (unpublished results, Tallon.) The ice-templated material is anisotropic similar to our material showing that we may have a more organized pore structure. Thermal diffusivity measured is 
lower than that of dense $\mathrm{TiB}_{2}$ at $35 \mathrm{~mm}^{2} \mathrm{~s}^{-1}$ measured by Munro ${ }^{14}$. Our porous $\mathrm{TiB}_{2}$ out performs $\mathrm{TiB}_{2}$ at a similar porosity produced from ice-templating, showing that our structure is more organized.

The third region can be seen above $600{ }^{\circ} \mathrm{C}$, where there is a change in slope. A hysteresis can be seen upon cooling pointing towards a permanent change in the material structure. The change in slope is result of a permanent chemical change in the material. The samples were run in a standard oxide furnace under argon. The atmosphere in the oxide furnace allowed for oxidation at a lower temperature. Kulpa et $\mathrm{al}^{114}$ found $\mathrm{TiB}_{2}$ powder to oxidize reversibly between $100-400{ }^{\circ} \mathrm{C}$ at $0.05 \mathrm{ppm} \mathrm{O}_{2}$. Andrievskii et al ${ }^{115}$ found the onset of oxidation to vary between $210-475^{\circ} \mathrm{C}$ based on particle size between $75-1500 \mathrm{~nm}$ respectively. Studies on oxidation of dense $\mathrm{TiB}_{2}$ show oxidation between $800-1000^{\circ} \mathrm{C}^{116,117}$. Our material experiences the onset of oxidization above $400^{\circ} \mathrm{C}$ because we have a partially sintered material. However, the oxidation is worse than the dense material because of the open pores allow for transport of oxygen into the material. When $\mathrm{TiB}_{2}$ oxidized to $\mathrm{TiO}_{2}$ the anisotropic pore structure is lost and the conductivity will be significantly decreased. Dense $\mathrm{TiO}_{2}$ has a thermal conductivity of 2 $W m^{-1} K^{-1}$ at $200{ }^{\circ} C^{118}$. The change in slope to a sharper decease in conductivity is a result of the oxidation of $\mathrm{TiB}_{2}$ to $\mathrm{TiO}_{2}$. This change in conductivity is not a reversible change since it is taken above $400^{\circ} \mathrm{C}$, resulting in a hysteresis upon cooling ${ }^{114}$. 
a)

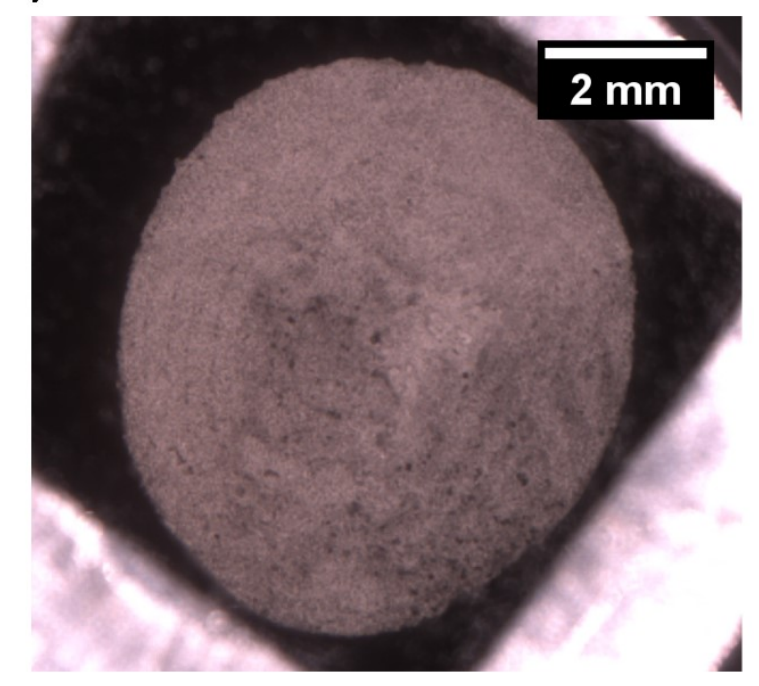

c)

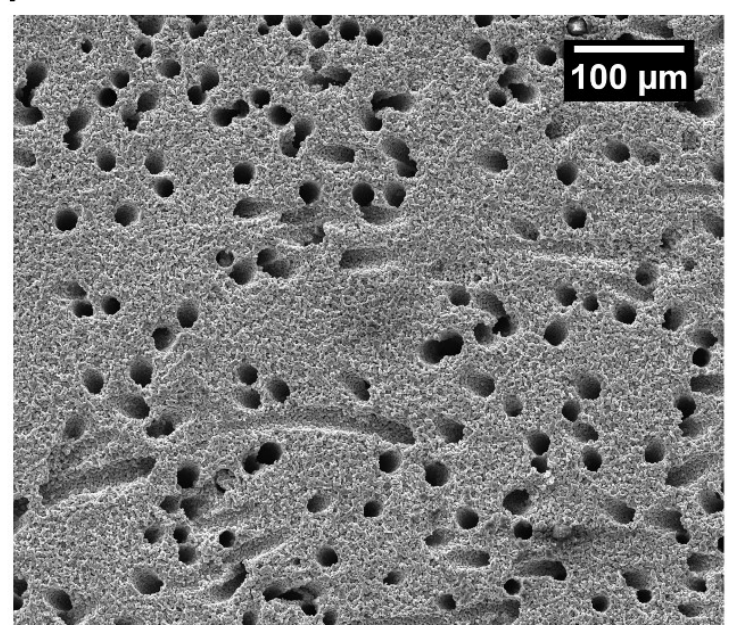

b)

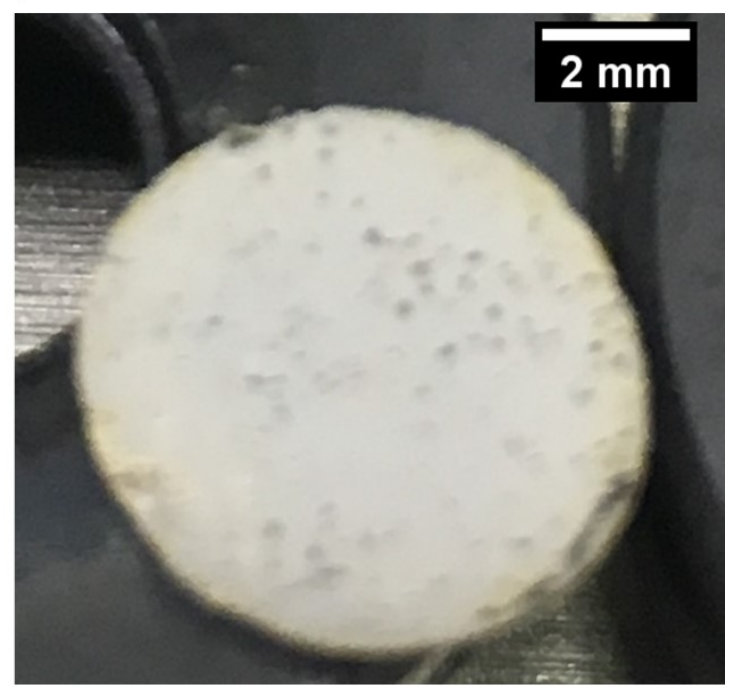

d)

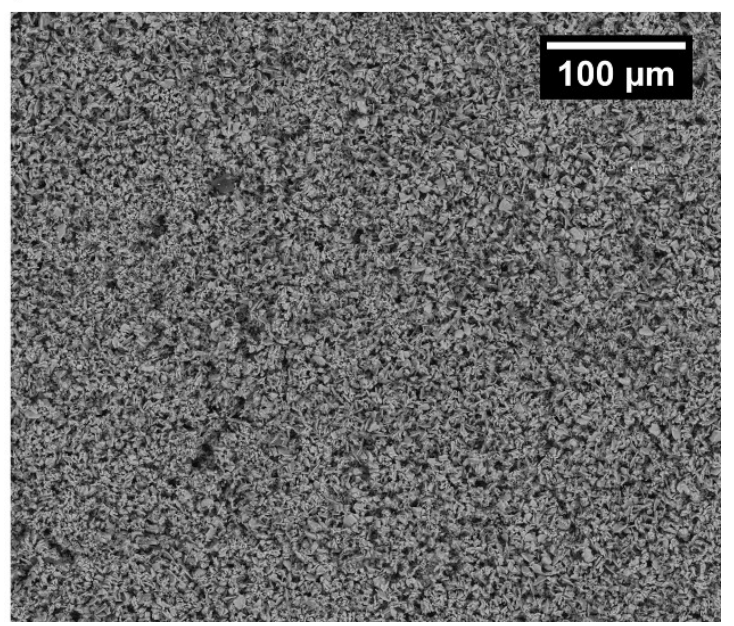

Figure 25: Change in microstructure and appearance from left) un-tested $\mathrm{TiB}_{2}$ to right) tested $\mathrm{TiB}_{2}$

Figure 25 illustrates a definite change in material appearance and a change in microstructure.

Further analysis was done using SEM with EDS capabilities. The EDS was able to show near

stoichiometric amount is $\mathrm{Ti}$ and $\mathrm{B}$ in the non-tested sample and $\mathrm{Ti}$ and $\mathrm{O}$ in the tested sample.

In addition to the dominant materials of $\mathrm{TiB}_{2}$ and $\mathrm{TiO}_{2}$ found in the non-tested, and tested

samples respectively $\mathrm{Cu}, \mathrm{Co}, \mathrm{Sn}$, and $\mathrm{C}$ were present in small quantities from the samples 
cutting with a diamond saw. The change was severe such that the cylindrical pores were completely covered by $\mathrm{TiO}_{2}$ formation.

\subsubsection{Thermal Model Comparison}

The materials tested have several types of porosity a) aligned macro-porosity from the sacrificial filler, b) interparticle porosity from partial sintering and c) an additional small amount of porosity due to processing defects. Aligned porosity can be approximated from the parallel approach where thermal conductivity is treated like electrical resitivity ${ }^{20}$. It is assumed that the aligned macro-porosity represents a constant $20 \mathrm{vol} \%$ porosity, (calculated with Equation 4.) It is important to note that the parallel approach does not consider the pore diameter. Interparticle porosity makes up a constant $30 \mathrm{vol} \%$ of the struts. The partially sintered material requires a slightly more complex approximation to include the effect of particle necking, (calculated with Equation 5.) The last amount of porosity is variable and from defects arising during improper filling during casting and fiber burnout, (fit with Equation 6.) 


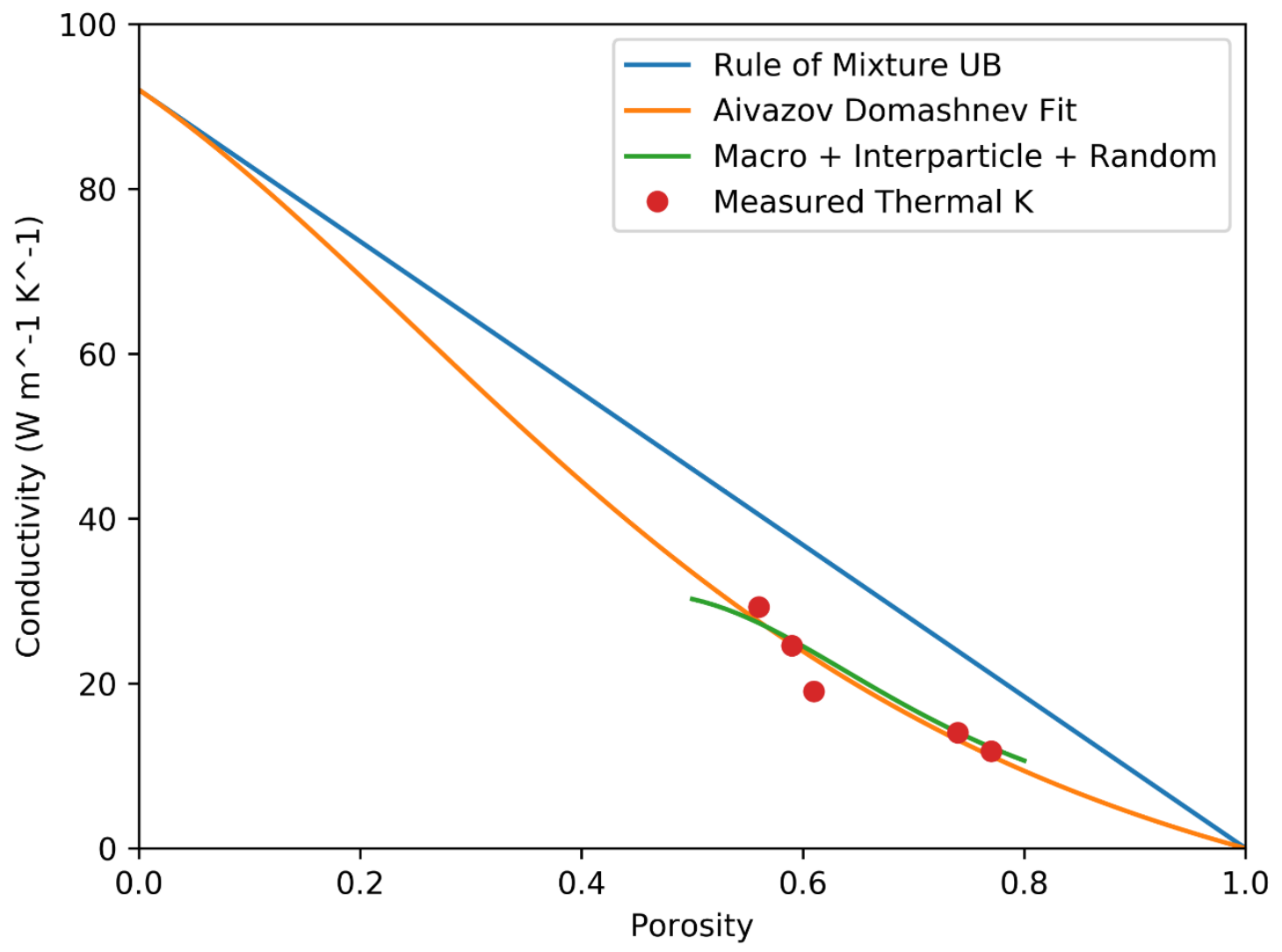

Figure 26: Thermal conductivity vs porosity plotted with several models for accurate fitting of the measured thermal conductivities.

Figure 26 shows the $\mathrm{k}$ values as a function of porosity, calculated using the rule of mixtures, the partial sintering model, Aivazov Domashnev fit, and combine model. Equation 11 to Equation 13 shows the combined model.

Equation 11: Step 1 to application of combined model

$$
k_{\text {eff } 1}=k_{\text {partially sintere }}=k_{\sigma} * \frac{2 *(1-P)}{\pi * r}\left(\frac{0.56}{x}+\frac{8 * r *\left(1-\frac{r}{r+r_{m}}\right)}{\pi *\left(x+r \frac{x+r_{m}}{r+r_{m}}\right)^{2}}\right)^{-1}
$$


Equation 12: Step 2 to application of combined model

$$
k_{\text {eff } 2}=k_{\text {upper bound }}=k_{\text {eff } 1} *(1-P)+k_{\text {pore }} * P
$$

Equation 13: Step 3 to application of combined model

$$
k_{\text {combined }}=k_{A-D \text { fit }}=k_{\text {eff } 2} * \frac{1-P}{1+n * P^{2}}
$$

The combined model has potential for accommodation to changes in any of the three pore structures observed. All of the model inputs and fitting parameters are summarized in Table 8. The fit parameters differ in the two applications of the Aivazov-Domashnev equation because $n$ is empirically associated with a particular material and pore structure ${ }^{94}$. The use of the AivazovDomashnev in the combined model represents a portion of the pore structure while in the application of the equation for the total porosity all structures are included.

The conductivity would be near the upper bound if all the porosity was aligned. The partially sintered structure and the residual pores lower the conductivity from the upper bound. The partially sintered approximation lies below the measured data but fits reasonably well showing that the partially sintered structure has a large contribution to the composite conductivity. The Aivazov-Domashnev approximation for random porosity fits well with the conductivity data but does not provide insight into the various structural contributions to the composite conductivity. 
Table 8: Input parameters for thermal conductivity models.

\begin{tabular}{|c|c|c|}
\hline \multicolumn{3}{|c|}{ Partially Sintered } \\
\hline$k_{\sigma}=$ & $92.015\left(\mathrm{~W} \mathrm{~m}^{-1} \mathrm{~K}^{-1}\right)$ at $200^{\circ} \mathrm{C}$ & Intrinsic Material Conductivity \\
\hline$P=$ & Independent variable & Porosity \\
\hline$r=$ & $1.5 \mu \mathrm{m}$ & Particle Diameter \\
\hline$x=$ & $0.918 \mu m$ & Particle Neck Radius \\
\hline $\mathrm{r}_{m}=$ & $0.18 \mu m$ & Neck Radius of Curvature \\
\hline \multicolumn{3}{|l|}{ Upper Bound } \\
\hline$P=$ & Independent variable & Porosity \\
\hline$k_{\text {pore }}=$ & $0\left(W \mathrm{~m}^{-1} \mathrm{~K}^{-1}\right)$ below $1000^{\circ} \mathrm{C}$ & Pore Thermal Conductivity \\
\hline \multicolumn{3}{|c|}{ Aivazov-Domashnev Fit } \\
\hline$P=$ & Independent variable & Porosity \\
\hline$n=$ & $\begin{array}{l}11 \text { (combined model) } \\
1 \frac{1}{2} \text { (Independent model) }\end{array}$ & Fit variable \\
\hline
\end{tabular}

The thermal conductivity data fits well with the Aivazov-Domashnev fit and well with the combined model. The current application of the combined model approximates the change in defect porosity, but does well to predict the conductivity of the system with few defects at 50 vol $\%$ without any fit parameters before the Aivazov-Domashnev fit comes into play. The accuracy of the models prediction at $50 \mathrm{vol} \%$ shows the strong influence of the interparticle porosity on thermal conductivity. Conductivity would be much lower if measured in the other direction. Ogushi et al ${ }^{91}$ estimated a $40 \%$ decrease in thermal conductivity between conductivity parallel to perpendicular aligned pores using EMF theory, and found good agreement with experimental data for aligned porous copper at $40 \mathrm{vol} \%$ porosity. Hu et al ${ }^{21}$ saw a $70 \%$ decrease in conductivity in the parallel vs perpendicular conductivity of freeze cast yttria-stabilized zirconia at 73-78 vol \% porosity. We can expect a similar type of behavior for the anisotropy of our material, but a slightly less pronounced effect due to the interparticle porosity and defect porosity. 


\subsubsection{Mechanical Behavior}

a)

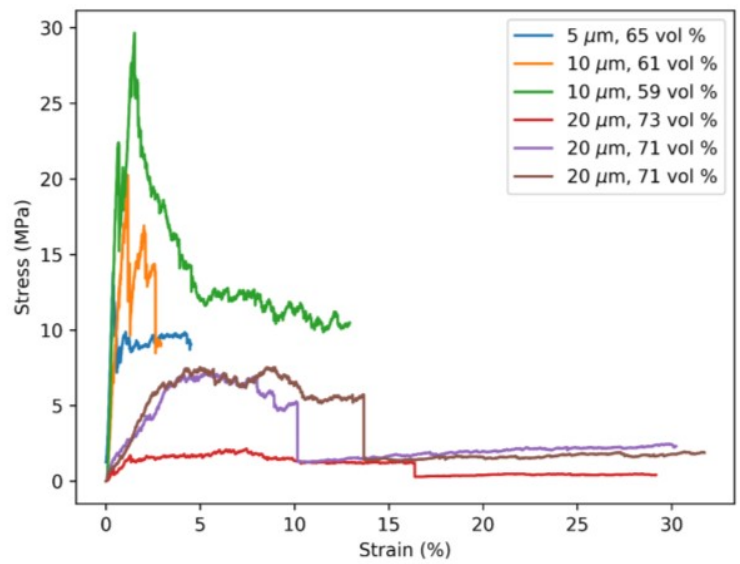

b)

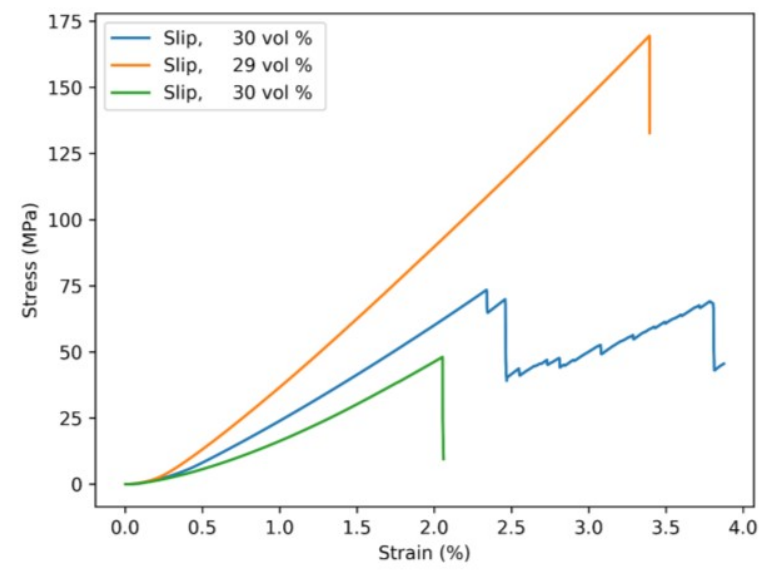

Figure 27: Mechanical Behavior a) aligned porous samples with progressive crushing behavior and b) slip cast samples with brittle like behavior.

Figure 27 shows the results of compression tests along the pore direction for samples with different porosity and pore size, as well as for slip cast samples representing the material in the struts. All the aligned porous ceramic materials experienced progressive crushing, with multiple fracture periods before complete failure. The slip cast samples had significantly greater strength, and more pronounced brittle behavior. The progressive crushing samples showed a less smooth loading and after the initial failure they continued to support load to strain values of 2-5 \% as the sample was further crushed. Cracks form during loading as localized stresses are developed, but because of the increased porosity cracks will not propagate till catastrophic failure. This progress crushing from multiple crack but not complete failure result in a rounded sloping curve similar in appearance to a ductile material. Seuba et $\mathrm{al}^{25}$ postulated that micro cracks parallel to the maximum loading resulted in the catastrophic brittle failure, while the 
progressive crushing samples failed due to buckling stress at the midpoint of the sample. The compressive strength correlates better with percent porosity then pore size.

The brittle samples showed a smooth increase in load with displacement and a single failure point. Although the slip cast samples are porous they show brittle behavior because of the interconnectivity of the ceramic is higher and cracks propagate till catastrophic failure. Partially sintered alumina with density above $63 \mathrm{vol} \%$ studied by Hirata et al ${ }^{119}$ showed similar brittle behavior under compression.

Failure is determined by stress concentrations and crack propagation, initiating in the mid plane where bending stresses are greatest. Stress concentrations are located in interparticle pores, non-aligned cylindrical pores and in defect pores from casting and potentially burnout. Each sample regardless of fiber size will have comparable interparticle porosity, while the cylindrical pores from the fibers will vary based on fiber size. In addition to the two controlled forms of porosity, some defect pores exist and are coupled in the total porosity. The largest pores will have the greatest stress concentrations, therefore the filler burnout defects as well as nonaligned cylindrical pores are the most likely sources for failure. Greater understanding of the defects that arise in filler burnout is necessary to further understand the effect of pore size parallel to loading.

Materials in Figure 27a show progressive crushing behavior typically associated with porous structures, while materials in Figure $27 \mathrm{~b}$ show a more typical brittle behavior and fracture mode. Seuba et al ${ }^{25}$ showed this progressive crushing behavior in porous ice-templated alumina 
with $72 \mathrm{vol} \%$ porosity, $60 \mathrm{vol} \%$ lamellar porosity from ice-templating and $12 \mathrm{vol} \%$ porosity from partial sintering.
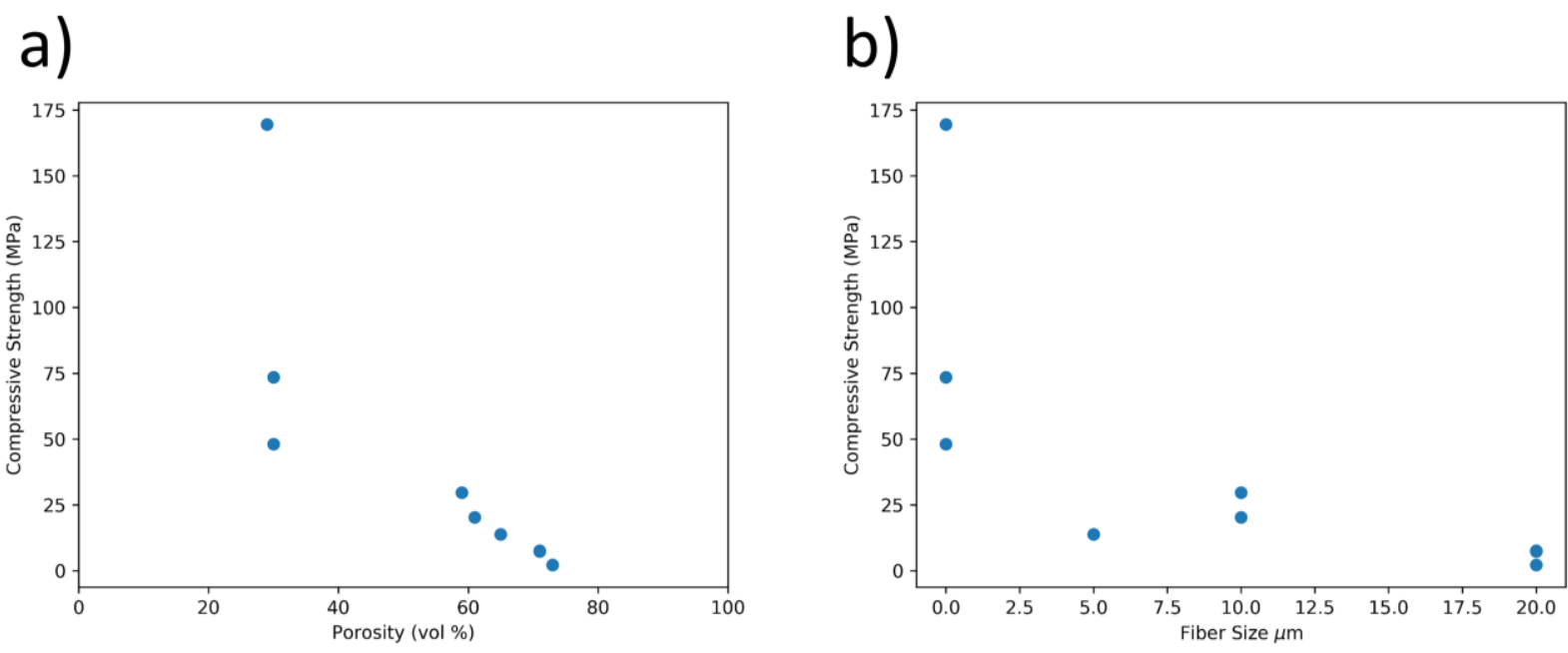

Figure 28: Compressive strength trends vs a) porosity and b) fiber size

Compressive strength shows a strong correlation with porosity. Figure 28a shows a visible trend of decreasing compressive strength with increasing porosity. Dense $\mathrm{TiB}_{2}$ has a compressive strength of $1.8 \mathrm{GPa}$ at $\mathrm{RT}^{14}$. Tallon et al, found the compressive strength for aligned $56 \mathrm{vol} \%$ porous $\mathrm{TiB}_{2}$ from ice-templating to be on the order of $155 \mathrm{MPa}$ with pore in the 30 to $100 \mu \mathrm{m}$ range ${ }^{22}$. This is much higher than our measured strength. The presence of defect pores from fiber burnout and casting could contribute to the lower strength. In the same work ${ }^{22}$, compressive strength vs porosity shows a trend for both $\mathrm{ZrB}_{2}$ and $\mathrm{TiB}_{2}$ across for multiple pore structures from 50 to $>90 \mathrm{vol} \%$ porosity, showing that porosity may have a stronger association with compressive strength than pore geometry in these multiscale porous UHTCs. Seuba et $\mathrm{al}^{25}$ plotted the compressive strength of alumina vs porosity and saw a decreasing trend. Cylindrical pore size does not show as strong a correlation to compressive strength, 
Figure 28b. For a real sample with a small percentage of non-aligned pores we would expect the compressive strength to decrease with increasing pore size. Increased pore size would mean that non-aligned pores have the potential to generate larger stress concentrations. To completely rule out the effect of pore size, a study of multiple pore sizes at a similar density would need to be conducted. After keeping density constant, the pore sizes may generate different flaws during burnout making the isolation of the pore size effect difficult. 
a)

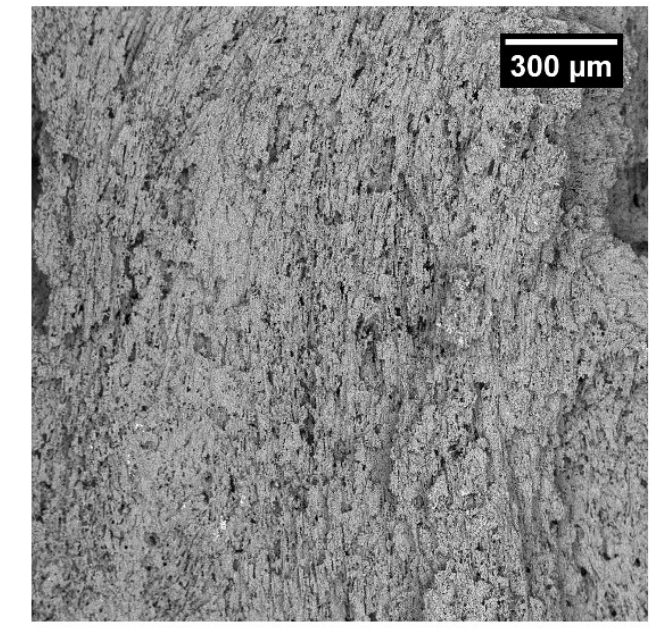

c)

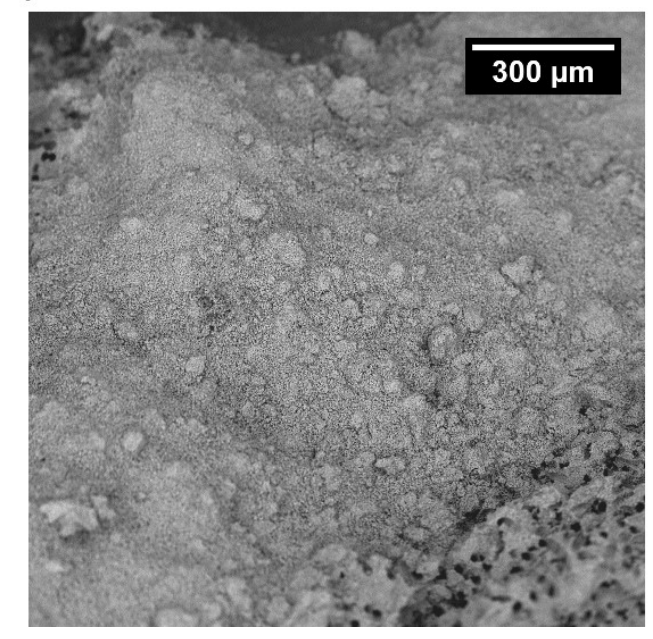

b)

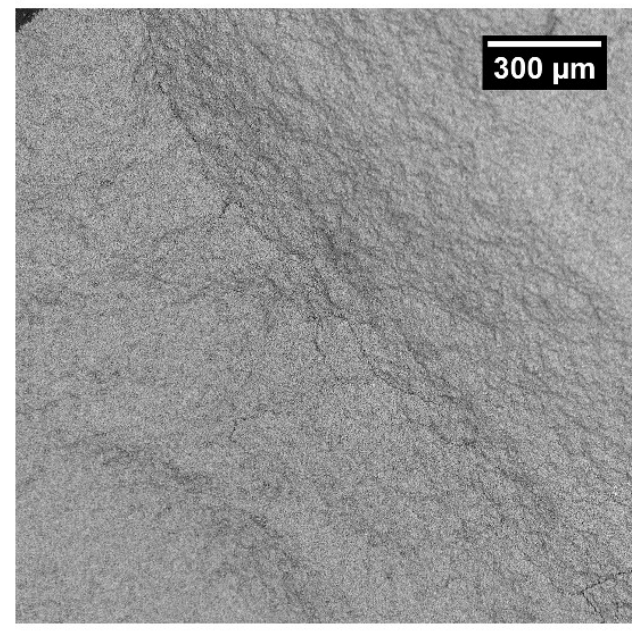

d)

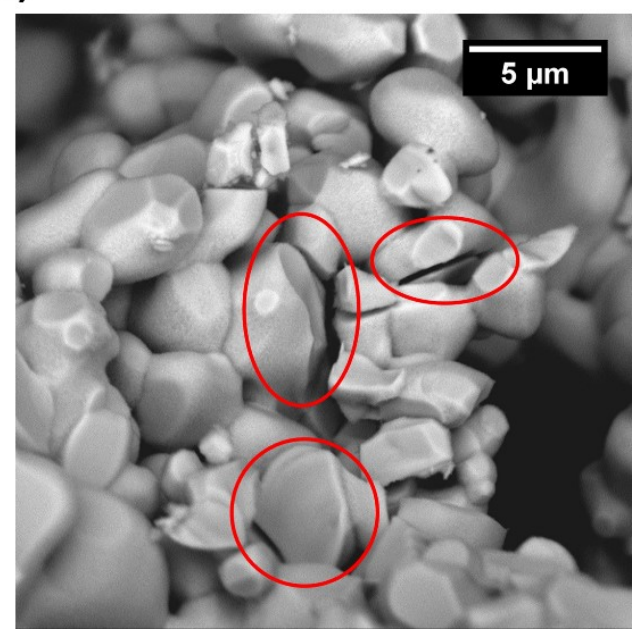

Figure 29: Fracture surfaces from compression test a) surface parallel to loading $10 \mu \mathrm{m}$ cylindrical pores and 61 $\%$ porous, b) surface parallel to loading of slip cast sample, c) surface normal to loading of $\mathbf{2 0} \mu \mathrm{m}$ and d) instances of inter-granular fracture.

Figure $29 \mathrm{c}$ shows fractured surfaces of samples after mechanical testing. The fracture surface parallel to loading direction, Figure 29 a, was distinct form the surface perpendicular to loading, Figure $29 \mathrm{~b}$. The surfaces shown are representative of the fracture planes for all progressive crushing samples. The crack propagation mechanism appears to be similar to the mechanism described by Meille et al ${ }^{107}$ as "highly porous, cellular like fracture" where the crack would span 
from pore to pore and not follow a rigidly straight path. Contrary to the brittle slip cast materials where there is a more rigidly flat fracture surface, Figure $29 \mathrm{~b}$. Similarly, there were few observed instances where a crack was seen to start in a pore, and not propagate. The surface normal to loading appeared like a powder compact where all the previously open cylindrical pores had been filled in with debris, Figure 29 c. There was evidence of intra-granular fracture shown in Figure $29 \mathrm{~d}$, but inter-granular fracture dominated the fracture surface. The fracture surface support the claim of progressing crushing as described by Seuba et al ${ }^{24}$.

a)

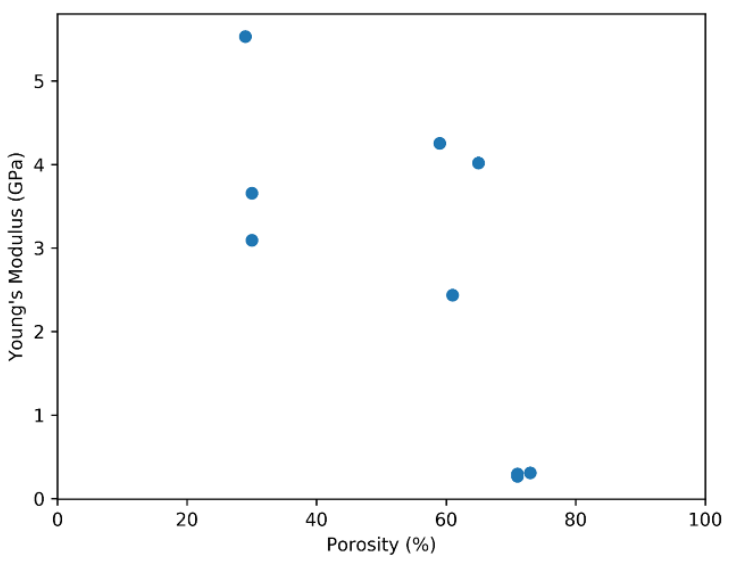

b)

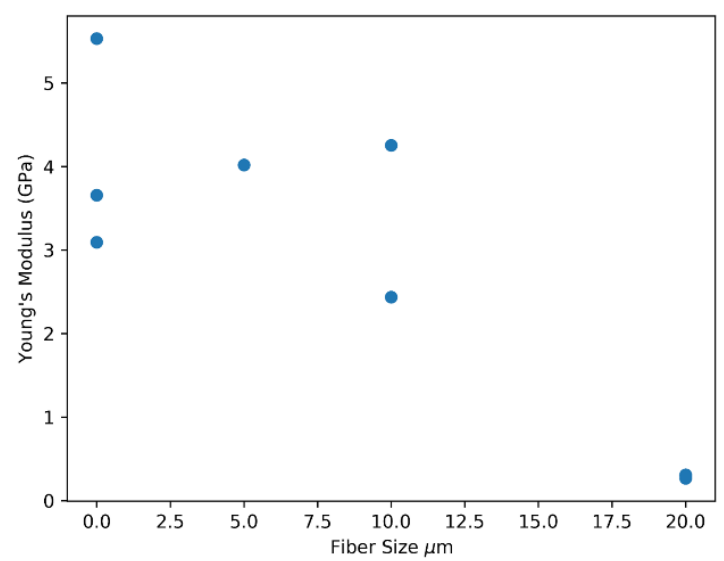

Figure 30:Young's modulus vs a) porosity and b) fiber size

Figure 30 shows Young's Modulus as a function of porosity vs pore size. Young's modulus decreases as porosity increases in Figure $30 \mathrm{a}$ and as fiber size increases in Figure $30 \mathrm{~b}$. The Young's modulus was calculated using the linear sections from Figure 27 during axial compression parallel to the pore direction. Dense $\mathrm{TiB}_{2}$ has a Young's modulus of $565 \mathrm{GPa}$, reported by Munro ${ }^{14}$. Porosity typically decreases the Young's modulus. For example, icetemplated $\mathrm{TiB}_{2}$ has a Young's modulus of $3 \mathrm{GPa}$ at $56 \mathrm{vol} \%$ porosity, as reported by Tallon et 
$\mathrm{al}^{22}$. Modulus describes the nondestructive loading of the material. Non-destructive deformation stems from a combined effect from the multiple porosities, therefore changing the geometry of one of those pores will change the modulus. The elastic behavior under loading is tied to the microstructure as a whole, the combined deformation and not the load limiting defect as is the case with compressive strength. Most literature and models for porosity as a function of Young's modulus are for partially sintered ceramics and will not behave the same as other porous microstructures, as noted by Chen et al ${ }^{106}$. Data of Young's modulus vs porosity from an alumina foam with different pore size could not be fit with a standard non-linear, likely because pore microstructure has a large effect ${ }^{95}$. The best understanding of Young's modulus will come from a combination of porosity and a microstructure geometric parameters. 


\subsubsection{Mechanical Model for Comparison}

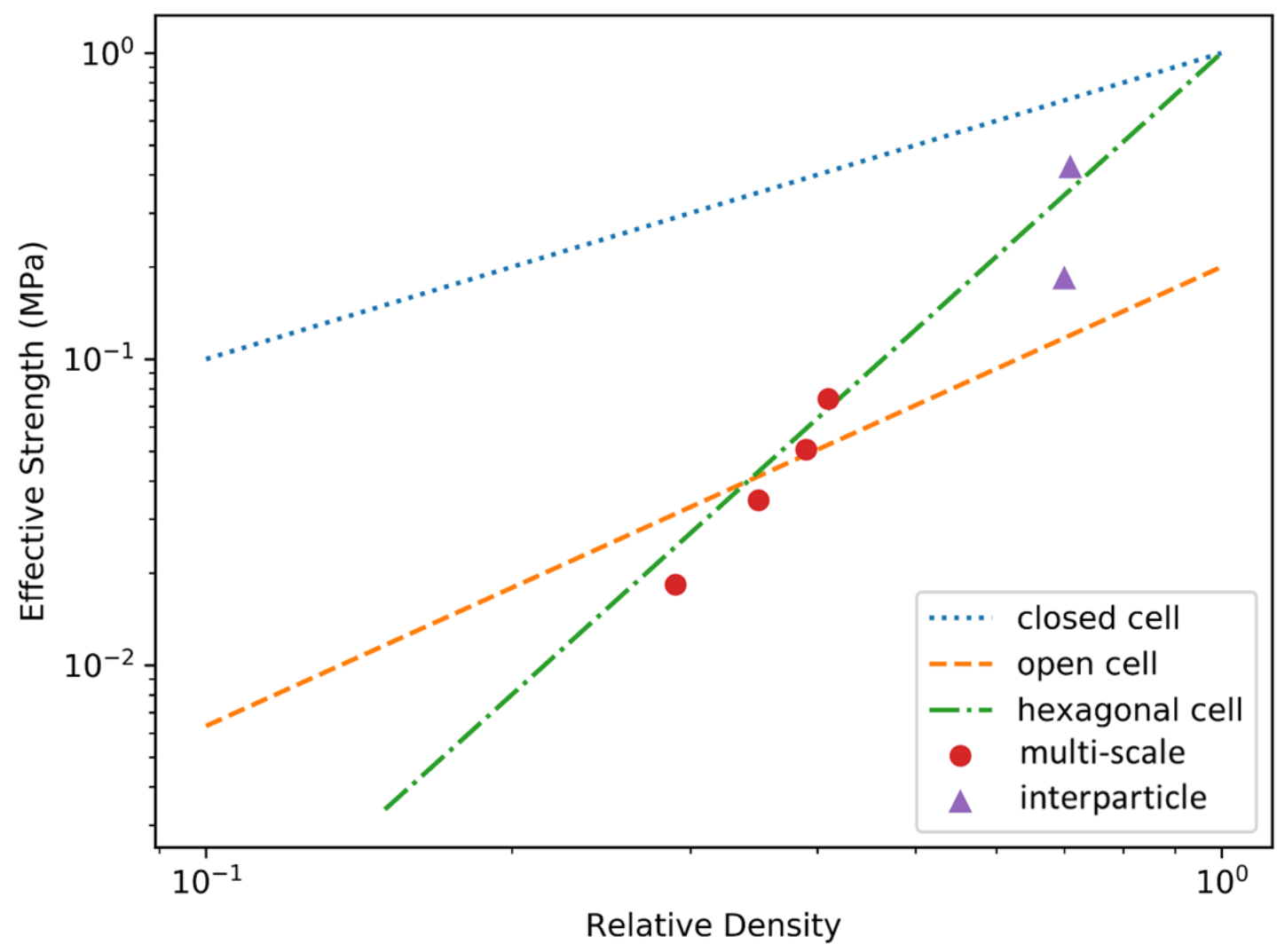

Figure 31: Gibson Ashby Models for Cellular Ceramics

Figure 31 shows the bounds of the Gibson Ashby models for open, closed and in-plane hexagonal cells plotted with the experimental data, corresponding to the porous samples and the slip cast samples. Relative compressive strength is plotted as a function of relative density. The aligned porous materials fall along the honeycomb prediction, but slightly below. The small discrepancy with the model can arise from two sources: first the walls of the aligned porous materials are partially sintered and therefore have less strength than a dense wall; and second, materials made with a sacrificial filler often show less strength then predicted due to defects 
from the filler burnout ${ }^{24}$. The current porosity level sits at the intersection where the out of plane honeycomb strength overcomes open cell structures. Above this intersection aligned porous materials will have a higher strength parallel to the pores compared to open cell isotropic structures.

\subsection{Conclusion}

The thermal and mechanical properties for aligned continuous porous $\mathrm{TiB}_{2}$ have been reported, showing that they fit well within the documented properties for multiscale porous $\mathrm{TiB}_{2}$ and that the organized structure has slightly increases the specific thermal conductivity. $\mathrm{TiB}_{2}$ from icetemplating showed better performance, likely due to issues with fiber burnout. Thermal conductivity was measured as a function of temperature ranging from $\mathrm{RT}-1000^{\circ} \mathrm{C}$, outperforming ice-templated $\mathrm{TiB}_{2}$ pointing to a better organized pore structure. The interparticle porosity from partial sintering had a large effect on the materials thermal conductivity. The compressive strength fit will well with the honey comb model by Gibson and Ashby. These multiscale porous structure fail due to progressive crushing with some instances of inter granular fracture. The data serves to inform the processing to refine the structure for varying applications, and to provide properties for aerospace designers to consider when, in the future, implementing this material structure. 


\section{Chapter 3: A Model for Thermal Characterization of UHTC Materials at Simulated Hypersonic Conditions}

\subsection{Introduction}

\subsubsection{Thermal Model Under Relevant Conditions}

In Chapter 2, the behavior of aligned multiscale porous $\mathrm{TiB}_{2}$ has been discussed for relatively isolated conditions. Hypersonic materials will experience a combined loading brought on by high heat fluxes, inducing rapid heating rates and disproportionate thermal expansion. Figure 32a shows the hypersonic environment around the leading edge, i.e. the flow properties governed by aerodynamic principles. The stagnation region where the highest convective heating takes place generates heat fluxes on the order of $800 \mathrm{~W} \mathrm{~m}^{-2} .{ }^{83} \mathrm{~A}$ shock layer arises from the stagnation point where high pressures will alter gas densities, change temperature and in some cases dissociate $\mathrm{N}_{2}$ and $\mathrm{O}_{2}$ creating a hyper reactive chemical environment ${ }^{83}$. Behind the shock layer, there is a boundary layer that will interact with a large area of the material behind the edge. Surface temperature in the boundary layer is a culmination of multiple effects such as re-radiation of the heat from the stagnation region into the environment, convention from the boundary layer into the material, and chemical heating from the enthalpy associated with material phase or composition change ${ }^{83}$. Figure $32 \mathrm{~b}$ shows the material state of a leading edge as a result of the hypersonic environment characterized by heat fluxes at the sharp tip, conduction from the tip, and re-radiation into the environment. Chen et $\mathrm{al}^{120}$ used a one dimensional finite difference model with surface temperature inputs from 
aerodynamic heating energy balance, and radiation to estimate material property requirements to prevent instability in the structural dynamics of a hypersonic vehicle frame based on temperature profiles from the unsteady heating environment. For proper design of hypersonic vehicles, it is imperative that the materials interaction with the hypersonic environment is precisely understood, and material properties uncertainties well defined.

a)

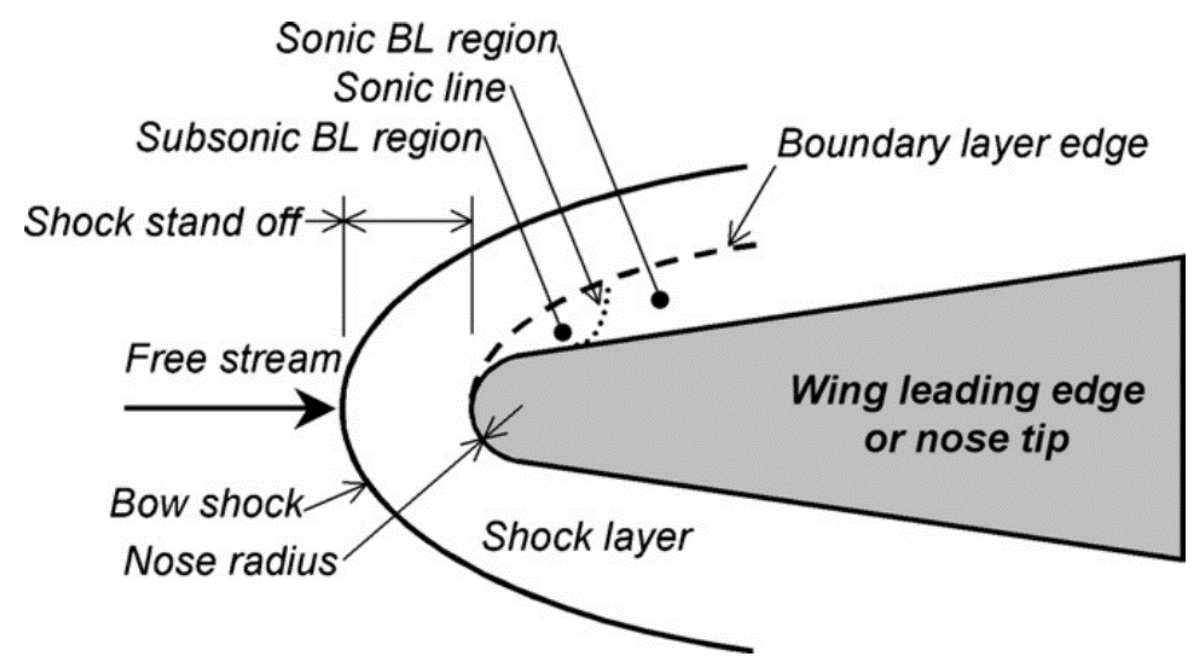

b)

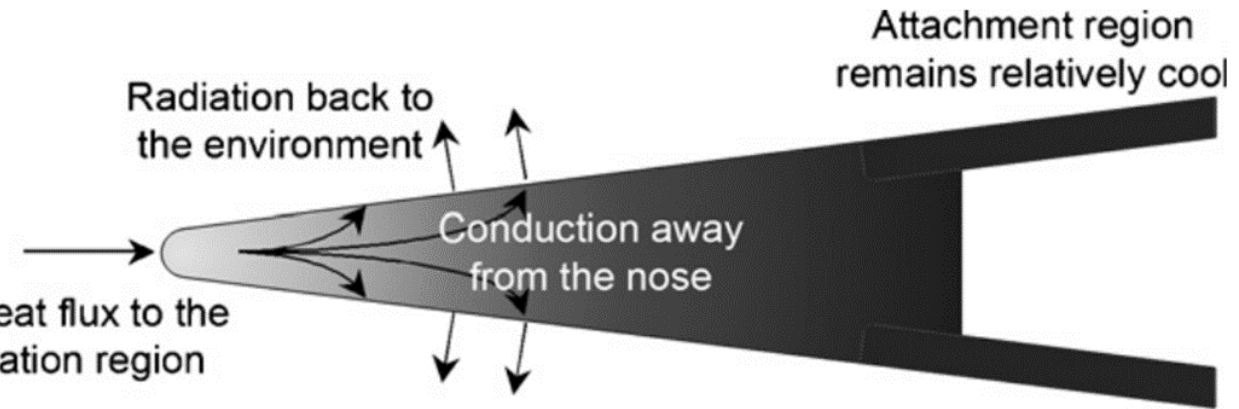

Figure 32: Breakdown of hypersonic flight taken from Squire et al "reuse with permission"

Recreating the hypersonic environment to measure material behavior and interaction is a necessary step in assessing the viability of candidate materials. Miller-Oana et al. stated in 2015 that "Currently, no single land based test facility is capable of duplicating all aspects of the 
extreme hypersonic flight environment."121 That being said, different tests can excel in creating specific subsets of hypersonic conditions, as shown in Table 9. The difficulty in all hypersonic tests is to simultaneously create harsh conditions while having an exact knowledge of the environment created to extract accurate material properties. Oxyacetylene tests are capable of generating suitably harsh environments at a fraction of the cost of Arcjet tests and Scramjet tests, making oxyacetylene tests useful for the screening of large sample sets, specifically in a research lab setup. The limitations of an oxyacetylene torch test are in subsonic gas velocity and $\mathrm{pO}_{2}$. However, oxyacetylene is still able to provide one of the highest heat fluxes, after Arcjet testing.

Table 9: Table of hypersonic characterization technique capabilities taken from Miller-Oana et al ${ }^{121}$.

\begin{tabular}{|c|c|c|c|c|}
\hline Test facility & Heat Flux $\left(W m^{-1} K^{-1}\right)$ & Flame Type & $\mathrm{pO}_{2}(k P a)$ & Velocity $(m / s)$ \\
\hline Arcjet PWT & $285-350$ & Plasma & $17-46$ & $\sim 9000$ (supersonic) \\
\hline ICP torch & $75-125$ & Plasma & $\sim<10$ & 88-136 (subsonic) \\
\hline $\begin{array}{l}\text { Scramjet } \\
\text { testing }\end{array}$ & $200-1800$ & Combustion & $\sim 19$ & $\begin{array}{l}250-4000 \text { (sub to } \\
\text { supersonic) }\end{array}$ \\
\hline $\begin{array}{l}\text { Oxyacetylene } \\
\text { torch }\end{array}$ & $\begin{array}{l}148-532 \\
830_{\max }\end{array}$ & $\begin{array}{l}\text { 1.35 VFR } \\
\text { combustion } \\
\text { (neutral) }\end{array}$ & $\begin{array}{l}1.4-7.2 \\
0.68_{\min }\end{array}$ & $\begin{array}{l}\sim 200 \text { at torch tip } 50 \\
\text { at } 10 \mathrm{~cm}\end{array}$ \\
\hline $\begin{array}{l}\text { Oxyacetylene } \\
\text { torch }\end{array}$ & $130-464$ & $\begin{array}{l}\text { 1.5 VFR } \\
\text { combustion } \\
\text { (oxidizing) }\end{array}$ & $2.3-9.6$ & $\sim 200$ at torch tip \\
\hline $\begin{array}{l}\text { Oxyacetylene } \\
\text { torch }\end{array}$ & $112-439$ & $\begin{array}{l}\text { 1.7 VFR } \\
\text { combustion } \\
\text { (oxidizing) }\end{array}$ & $3.6-11.3$ & $\sim 200$ at torch tip \\
\hline
\end{tabular}

Oxyacetylene tests have been used to screen UHTC candidate materials in many instances $44,50,51,122-125$. An oxyacetylene torch test uses specific ratios and flow rates of oxygen and acetylene to produce a range of conditions from oxidizing to neutral high heat flux.

Oxyacetylene torch tests have been traditionally utilized in a screening "go/no go" capacity, with post-test quantification of oxidation. During testing the temperature of the sample face is 
measured to record conditions and correlate to post-test analysis. Most tests quantify the oxidation and ablation through post-test mass change, and SEM with EDS of the materials surface to determine oxidation thickness and composition. For example, Han et al ${ }^{126}$ characterized $\mathrm{ZrB}_{2}-\mathrm{SiC}$ composites under a $2200{ }^{\circ} \mathrm{C}$ flame and used SEM and mass loss measurements to characterize the oxidation behavior of mass loss at $-0.23 \mathrm{mg} \mathrm{s}^{-1}$ and an oxidation rate of $0.66 \mu \mathrm{m} \mathrm{s}^{-1}$.

The study of porous UHTCs under and oxyacetylene flame are less prevalent then their dense counter parts. Porous materials are most often used as insulation, and only directly exposed to hypersonic flow when applied as ablatives. There are several cases of porous materials for hypersonic ablatives mostly of the material carbon/carbon-ZrC-SiC ${ }^{123,127-130}$. Xie et al ${ }^{123}$ studied the ablation behavior and oxide formation of carbon/carbon-ZrC-SiC composite at temperatures upwards of $3000{ }^{\circ} \mathrm{C}$ with varying porosity up to $20 \%$ and reported linear and mass ablation rates of $2.48 \times 10^{-3} \mathrm{~m} \mathrm{~s}^{-1}$ and $3.75 \times 10^{-4} \mathrm{~g} \mathrm{~s}^{-1}$ respectively. Qualitative tests and post-test oxidation analysis are useful, but understanding and controlling the heat distribution within a material during a test would allow for a better understanding of the material behavior. 


\subsection{UHTC Characterization Rig}

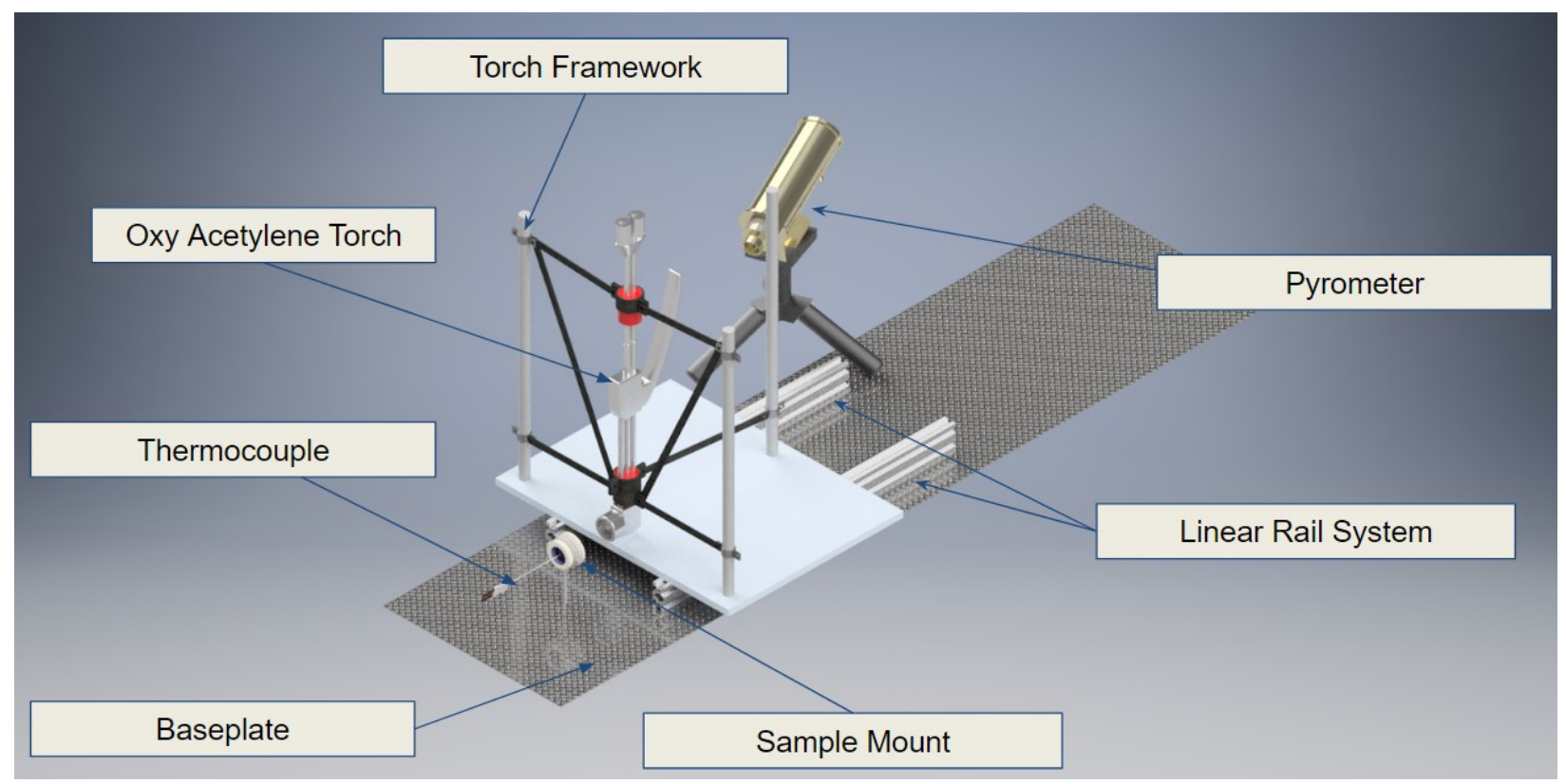

Figure 33: CAD of oxyacetylene torch rig setup

An oxyacetylene torch rig has been designed to provide both a screening test and oxidation characteristics for UHTC candidate materials as well as provide an idea of effective properties, in this case thermal conductivity. The design focus on the sample holder to exercise improved control over the test materials heating. Figure 33 shows the setup of our characterization rig which based on the general structure commonly implemented in literature ${ }^{51,126,127}$. In our design, there is an oxyacetylene torch on an automated linear actuating rail system to control distance from the flame to the sample surface (typically $15-30 \mathrm{~mm}$,) a pyrometer to measure sample front face temperature, a thermocouple to measure sample back face temperature, and a specially designed sample mount. 


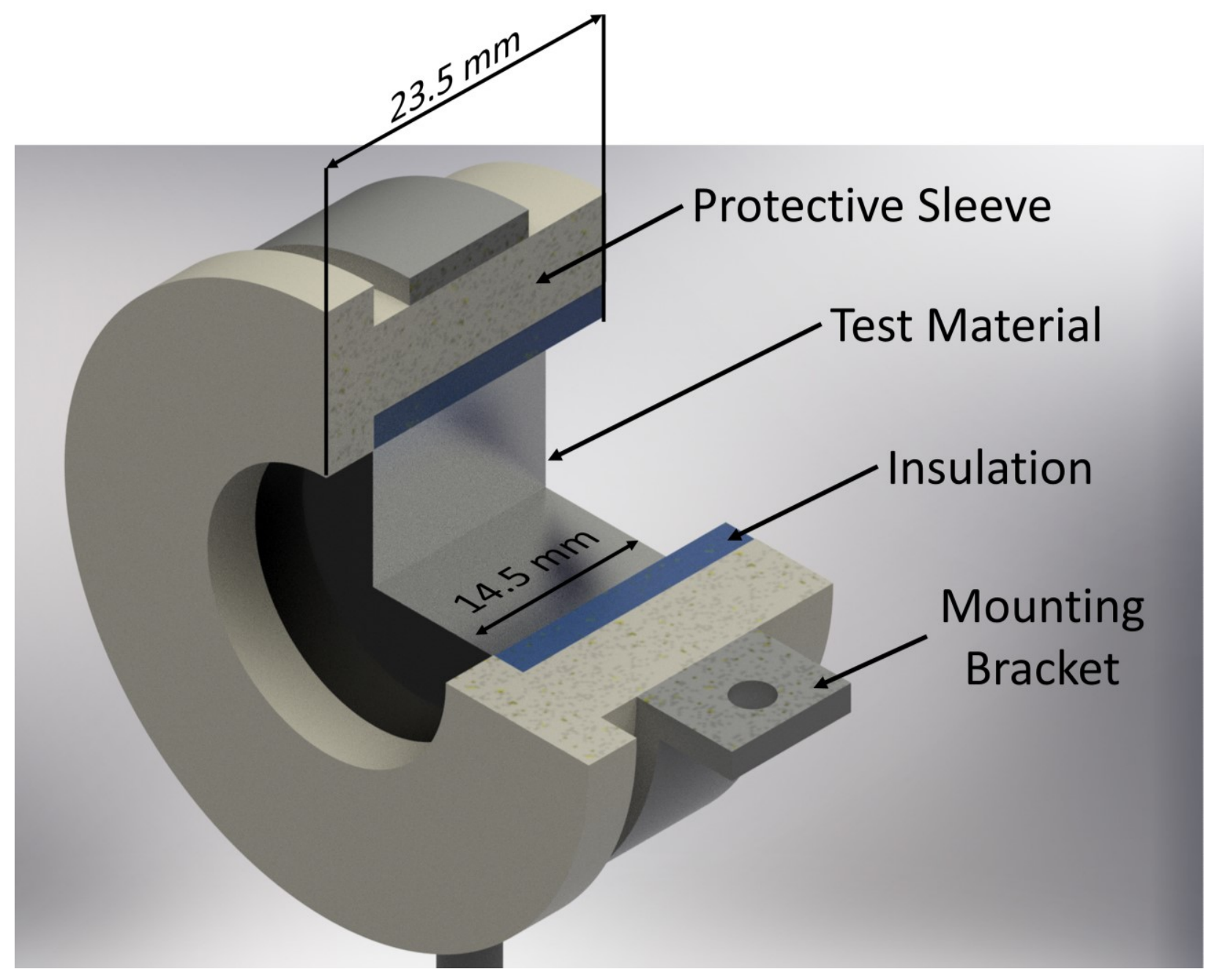

Figure 34: Sample holder for UHTC rig designed to allow for known material heat distributions.

Figure 34 shows a schematic of the sample holder which will help remove uncertainty from the material's thermal interaction with the torch. The sample holder is composed of an insulating dense material outer sleeve (alumina) and an inner layer of graphite felt. The dense outer serves to limit radial heat flow while the graphite felt bridges the gap between the sample and outer sleeve. Limiting heat flow to one direction allows for behavior similar to a larger area of material. Additionally, the graphite felt protects the sample from experiencing thermal stresses between the outer sleeve and sample. The dense outer layer has a lip to protect the felt from 
exposure to the oxidizing environment. With this small modification to the traditional rig set up we expect to retrieve thermal conductivity during the oxyacetylene torch test, using a thermal model.

\subsection{Thermal Profile Model}

An unsteady state thermal model was developed to estimate the thermal state of the material during heating. The thermal model serves two functions: 1 ) to estimate the test length and thermal profile of a material before a testing can occur, to more accurately determine test conditions at high temperature and safety precautions and 2) to back calculate an effective thermal conductivity based on front face and back face temperatures measured during the test. The model will generate heat maps for the sample and sample holder over the projected test length to understand potential damage to the material and sample holder at high temperatures. A base model was written in house using Python to code a 2D finite difference mesh.

As discussed earlier there are a range of contributing sources to the heat, in the boundary layer. For simplicity, we assume heat transfer only happens through convection from the torch either to the material (or to the bulk or to any oxide that may develop during the course of testing), ignoring radiation and enthalpy contributions from chemical change. Conduction within the material is likewise under a simplifying assumption that heat capacity and thermal conductivity do not vary with temperature (in the range of 1800 to $2000^{\circ} \mathrm{C}$ ) and are set to constant values. Given these assumptions, our finite difference model will estimate the time for the sample and mount to reach steady state and give a heat map for each instance leading up to steady state. 
These values let us estimate the consumables needed for a test, confirm that our measurement equipment is adequate, check that safety precautions will be adequate over the given temperature ranges and to identify potential hot spots that will comprise the material or sample holder.

To calculate an effective thermal conductivity, we set the front face temperature value to a constant observed value from the test and then vary material conductivity until our model matches the measured temperature profile. The sources of error for this back-calculated effective thermal conductivity are primarily derived from our knowledge of the outer sleeve material properties and the convective transfer around the sample holder and back face of the material. These sources of error can be corrected for directly by gaining insight on the outer sleeve properties and convective transfer or indirectly by calibrating those properties by testing known materials with our rig and thermal model.

The model is based on a standard non-steady assumption using a 2D finite difference model written in python. The model will take the arguments of heat flux, sample thickness and diameter, as well as heat capacity, thermal conductivity, and density for the sample, felt and sleeve. 


\subsection{Model Description}

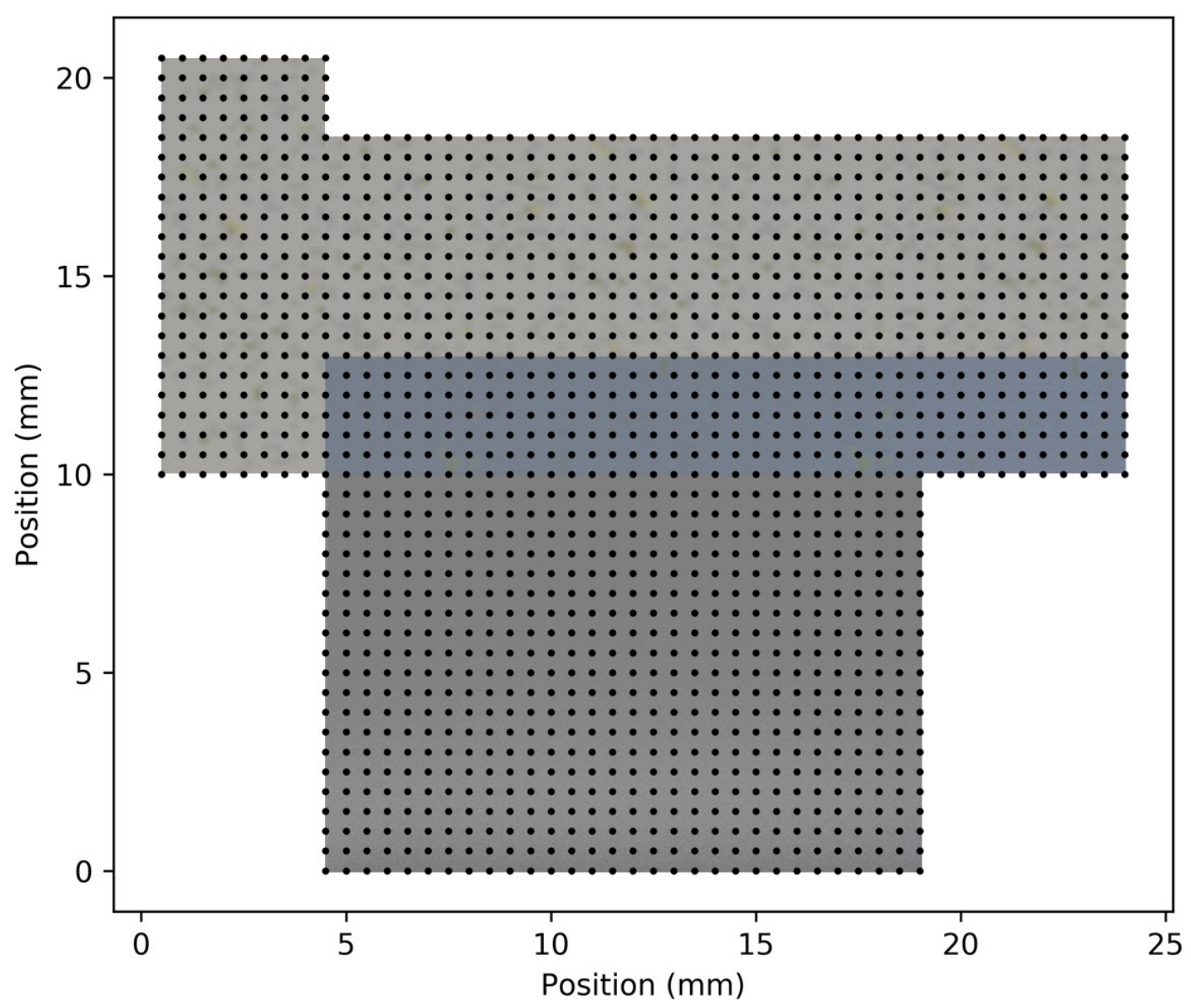

Figure 35: 2D finite difference node layout superimposed on cross-section of the sample holder.

A 2D finite difference model is used and herein the derivation of equations will be enumerated.

The finite difference model is built on the energy balance of a defined system of nodes given in

Figure 35 , with node size equal to $500 \mu \mathrm{m}$. The transport that will take place at a given node is a defined combination of convection and conduction, based on the location of each node within the material system. The conductive transfer is governed by Fourier's Law given in Equation 14. 
Equation 14: Fourier's Law for conduction in 1D.

$$
q_{x}=-k A_{x} \frac{d T}{d x} \text { and } q_{x}^{\prime}=-k \frac{d T}{d x}
$$

where $k$ represents the thermal conductivity and $q_{x}$ is the heat flux. Convective heat transfer is governed by Newton's Law of Cooling given in Equation 15.

Equation 15: Newton's law of cooling to describe convection.

$$
q_{x}^{\prime}=-h(\Delta \mathrm{T})
$$

$$
\text { where } \Delta \mathrm{T}=T_{s}-T_{\infty}
$$

where $h$ is the convective heat transfer coefficient and $\Delta \mathrm{T}$ represents the temperature difference between the material surface and the gas.

Heat accumulation for a given node will follow a conservation of energy such that the amount of heat entering equals the amount of heat leaving, assuming no radiation or heat generation:

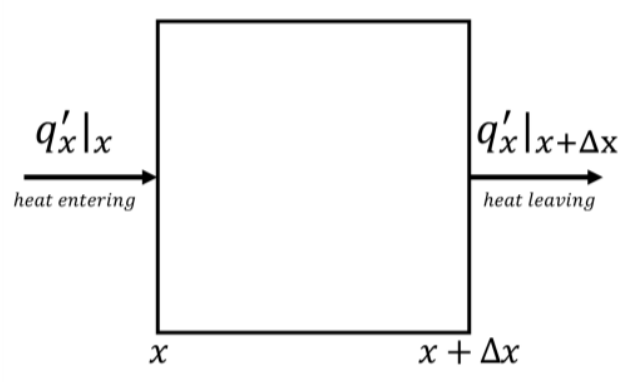

Figure 36: 1D conservation of energy visualization.

Equation 16: Conservation of energy in thermodynamic terms.

$$
\frac{\partial Q}{\partial t}=\left(\left.q_{x}^{\prime}\right|_{x}-\left.q_{x}^{\prime}\right|_{x+\Delta \mathrm{x}}\right) A_{\mathrm{yz}}+\left(\left.q_{y}^{\prime}\right|_{y}-\left.q_{y}^{\prime}\right|_{y+\Delta \mathrm{y}}\right) A_{\mathrm{xz}}
$$


with $A_{\mathrm{yz}}$ equal to the area in heat will pass through in the $y$ direction and $A_{\mathrm{yz}}$ in the $x$ direction.

Applying the first law of thermodynamics under constant pressure $\partial Q=d H$, where in heat equals change in enthalpy and rearranging the definition of heat capacity at constant pressure $C_{p}=\frac{1}{m}\left(\frac{\partial H}{\partial T}\right)_{p}$ to $\partial H=C_{p} m \partial T$, we get Equation 17.

Equation 17: Conservation of energy in terms of temperature and material properties.

$$
\begin{gathered}
C_{p} m \frac{\partial T}{\partial t}=\left(\left.q_{x}^{\prime}\right|_{x}-\left.q_{x}^{\prime}\right|_{x+\Delta \mathrm{x}}\right) A_{\mathrm{xz}}+\left(\left.q_{y}^{\prime}\right|_{y}-\left.q_{y}^{\prime}\right|_{y+\Delta \mathrm{y}}\right) A_{\mathrm{yz}} \\
\text { where } m=\rho V
\end{gathered}
$$

This allows us to derive change in temperature from the heat flux given by Fourier's Law of conduction and Newton's Law of Cooling based on the node conditions. Simplifying with equal node separation in $x$ and $y$.

Equation 18: Conservation of energy final form before implementation of conduction and convection equations.

$$
\frac{\partial T}{\partial t}=\frac{1}{C_{p} \rho V}\left(\left(q_{\text {in } x}\right) A_{\mathrm{xz}}-\left(q_{\text {out } x}\right) A_{\mathrm{xz}}+\left(q_{\text {in } y}\right) A_{\mathrm{xz}}-\left(q_{\text {out } y}\right) A_{\mathrm{xz}}\right) \text { with } \Delta \mathrm{x}=\Delta \mathrm{y}
$$

At this point the individual node equation will differ based on location within the test material and sample holder. There are 4 basic node conditions each with a heat flux defined in 4 directions. Table 6 summarized the heat flux for each direction for each node condition, as well as the rule for transfer between materials. The 2D finite difference method for modeling heat transport is well established, this derivation follows the work of Corcoran ${ }^{131}$. 
Table 10

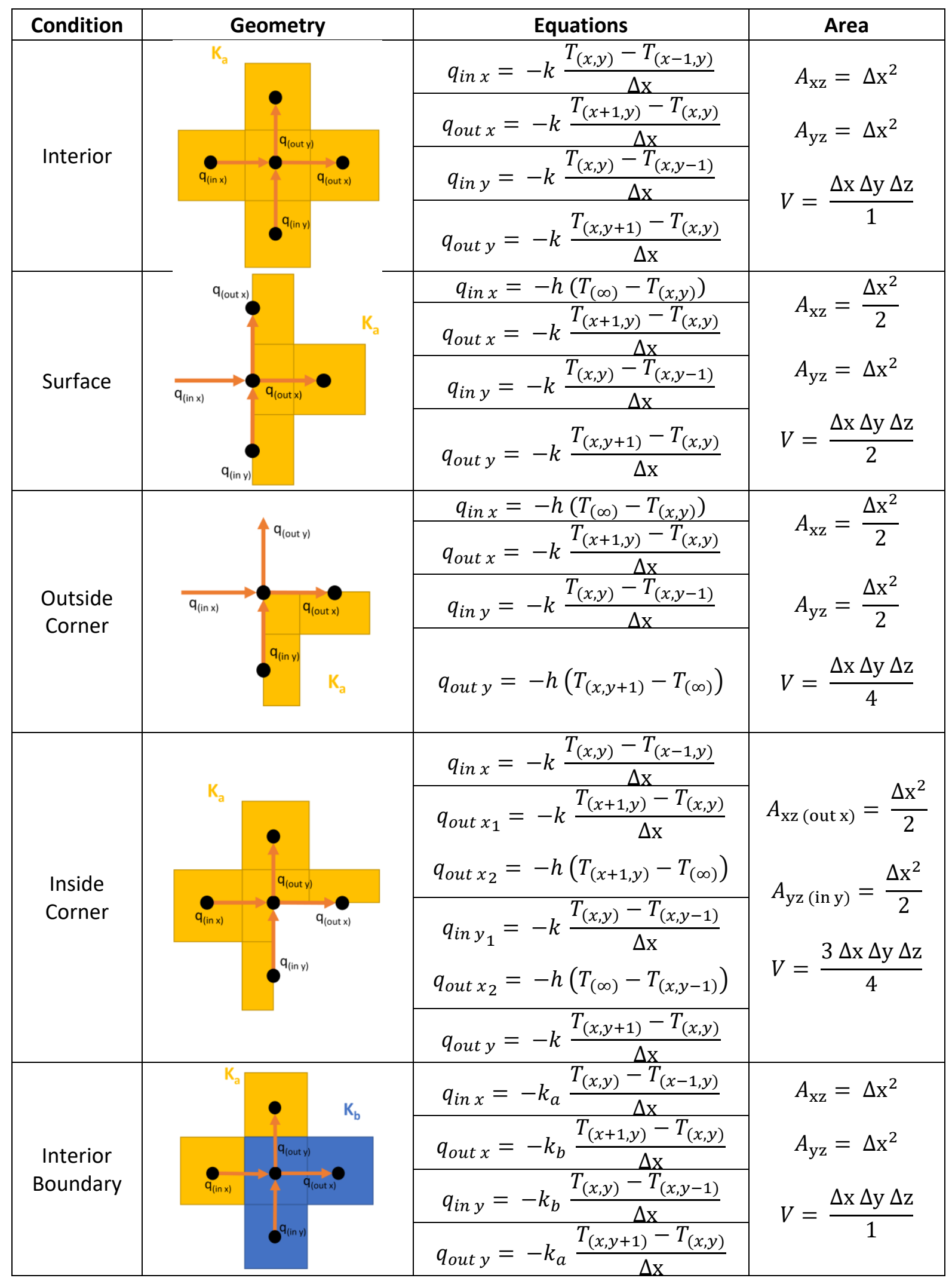




\subsection{Material Change During Testing}

Oxide formation is common under oxyacetylene tests and should be included, since the oxide often has significantly different thermal properties. When oxide forms it can be below the node resolution. For example, a dense $\mathrm{ZrB}_{2}$-SiC sample was shown to have a layer of oxidation 375 $\mu m$ thick after $10 \mathrm{~min}$ at above $2200^{\circ} \mathrm{C}{ }^{126}$. In this model, when the thickness of oxide formation is below the dimensions of the node resolution, we consider only convective heat transfer into the material and not conduction. Heat transfer at the surface is described by the convective heat transfer coefficient and the temperature drop. The convective heat transfer coefficient is material independent, but the heat flux will be divided by the density and heat capacity, of the material (bulk or oxide.) Oxide formation will have some effect on the heat entering a surface node, and that is accounted for in the finite difference model. If oxide thickness is above node resolution, then nodes will be modified to accommodate the properties related to the oxide. However, since this is not a common scenario, we will focus on oxide contributions below node resolution.

\subsection{Materials}

A porous aligned $\mathrm{TiB}_{2}$ with conductivities and densities analogous to that produced and tested in chapters 1-2 as well as bulk $\mathrm{TiB}_{2} \mathrm{HfB}_{2}$ and $\mathrm{ZrB} 2$ were chosen. These materials were chosen for their prevalence within the UHTC research community either as porous materials, composites or in bulk form and their range of thermal conductivities and densities, as well as our labs capability in producing these materials. All results described in the next section refer to model results. The model validation with experimental data is necessary, but out of the scope of the M.S. thesis. 
Table 11

\begin{tabular}{|c|c|c|c|c|}
\hline & $\mathrm{TiB}_{2}{ }^{\text {porous }}$ & $\mathrm{ZrB}_{2}{ }^{12}$ & $\mathrm{TiB}_{2}{ }^{14}$ & $\mathrm{HfB}_{2}{ }^{12}$ \\
\hline Thermal Conductivity $W m^{-1} s^{-1}$ & $10^{+}$ & 60 & 96 & 104 \\
\hline Density $\mathrm{g} \mathrm{cm}^{-3}$ & $1.9^{x}$ & 6.119 & 4.5 & 11.212 \\
\hline Heat Capacity $\mathrm{Jkg}^{-1} \mathrm{~K}^{-1}$ & $617^{x x}$ & 427 & 617 & 247 \\
\hline
\end{tabular}

porous aligned porous material characterized in Chapter 2.

${ }^{+}$Calculated from diffusivity measured by Oak Ridge National Lab

$x$ Measured using the Archimedes principle.

$x x$ Calculated following equation from Munro et al ${ }^{14}$.

These properties were taken from RT values. The material selection is meant to represent a distribution of properties within the bounds of UHTC materials and in order to compare the heating rates and temperatures with results from literature to validate our model. Additionally, we can get an effective value for the effect of porosity on thermal conductivity which can be difficult to estimate accurately.

\subsection{Results and Discussion}

\subsubsection{Beginning of Test}
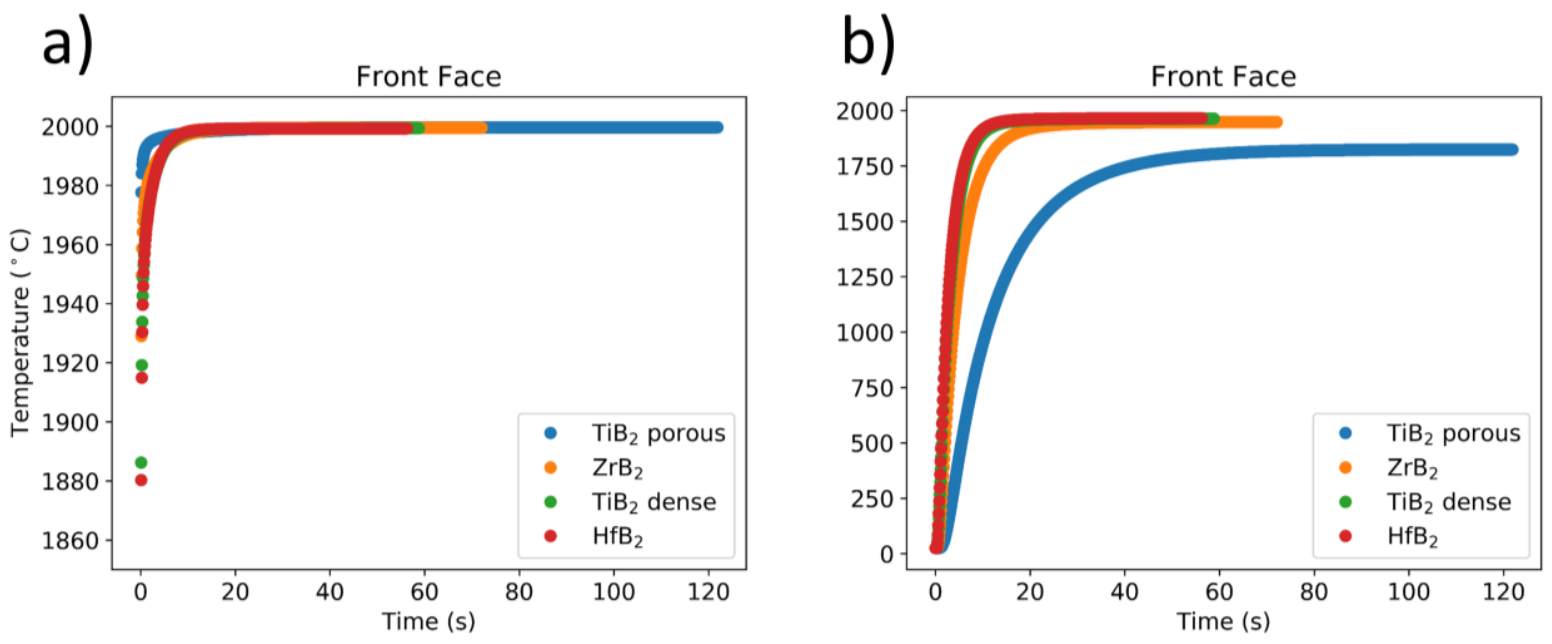

Figure 37: Evolution of temperature with time for a) front and b) back face of test material. 
Figure 37a shows the evolution of temperature with time for the center most node on the front face of the material, for the 4 test materials considered. The purpose of this plot is to show the time until equilibrium is reached. The heat buildup in the front face is dependent on the convective heat transfer, while the back face temperature depends on the bulk conductivity of the material. As expected, the higher conductivity samples $\left(\mathrm{HfB}_{2}\right.$ and $\left.\mathrm{TiB}_{2}\right)$ arrived at equilibrium within $60 s$, while the lower conductivity material took almost $80 s$, and the porous sample took $120 \mathrm{~s}$. The rate of temperature increase for the front face is based on the convective heat flux, which is a function of $h$ value, $C p$, and $\rho$. The porous $\mathrm{TiB}_{2}$ had the fastest increase in temperature of the front face, because of its lower density, and its lower capability to dissipate heat though conduction, due to a lower thermal conductivity.

The time to equilibrium for the front face depends strongly on the convective heat transfer coefficient $(h)$, which is a function of the flame's interaction with the material surface as is difficult to estimate. However, the $h$ value could be back calculated for a given system from the heat flux, material heat capacity and density. For increased accuracy regarding the front face time to equilibrium a more rigorous understanding of the flame heat profile and $h$ value profile is necessary. That being said, in most oxyacetylene torch testing cases the $h$ value is sufficiently high, on the order of $1,000,000 \mathrm{~W} \mathrm{~m}^{-2} \mathrm{~K}^{-1}$, such that it is not a limiting factor in the whole sample reaching equilibrium. Paul et al studied several UHTC materials (carbon/carbon composite, carbon-fiber/HfB 2 , and carbon-fiber $\left./ \mathrm{ZrB}_{2}\right)$, in a graphite holder at a heat flux of $\sim 15$ $M W \mathrm{~m}^{-2}$, all of which reached equilibrium of the front face temperature of 2100 to $2600{ }^{\circ} \mathrm{C}$ at around $30 \mathrm{~s}$. In a paper by Han et al it took upwards of $400 s$ for a $\mathrm{ZrB}_{2}-\mathrm{SiC}$ composite in a graphite sample holder to reach equilibrium at $2200^{\circ} \mathrm{C}$. Xie et al reported a time of $80 \mathrm{~s}$ for a 
carbon/carbon-ZrC-SiC composite to reach equilibrium temperature of $2400{ }^{\circ} \mathrm{C}$ at $10 \mathrm{~mm}$ from the flame. This variation across oxyacetylene tests shows that there are many system dependent variables to consider for heating rates for a test material. Dense graphite can vary in conductivity based on the manufacturing, and geometry of the sample holder will affect the system temperature gradients. Additionally, flame characteristics can change based on the nozzle type. The oxyacetylene torch system must be well characterized to estimate the heating behavior and sample holder geometry can have a large contribution. However, the effect of $h$ is not a limiting factor.

Ablation will also affect the surface temperature through enthalpic cooling as a result of the thermodynamics of the ablation process effecting the heat rate as the material approaches equilibrium. Miller-Oana et al ${ }^{121}$ studied several material groups (oxides, graphite, and diborides) in an attempt to decouple the effects of thermal conductivity and enthalpic cooling in thermal protection materials under an oxyacetylene torch. Oxide materials which have the lowest thermal conductivity and will not oxidize, showed the highest surface temperatures of $1600^{\circ} \mathrm{C}$. Graphite has a high conductivity and exothermic oxidation allowing it to remove heat faster. The graphite showed a surface temperature of $1472{ }^{\circ} \mathrm{C}$, both because of the high thermal conductivity and a shielding effect of the $\mathrm{CO}$ and $\mathrm{CO}_{2}$ oxidation products. $\mathrm{ZrB}_{2}$ and $\mathrm{ZrB}_{2-}$ $\mathrm{SiC}$ composites at $11 \mathrm{~cm}$, from the torch, with simultaneously high $\mathrm{pO}_{2}$ the diboride materials exhibited the lowest front face temperatures of $1320^{\circ} \mathrm{C}$. Miller-Oana cite the both high conductivity as well as enthalpic cooling from the endothermic reactions of oxide formation. At distances closer to the flame the diboride materials has a higher front face temperature, around $2000^{\circ} \mathrm{C}, 250^{\circ} \mathrm{C}$ above graphite and $150^{\circ} \mathrm{C}$ below the oxides, because of change in 
structure of the ablation products. $\mathrm{B}_{2} \mathrm{O}_{3}$ was evaporated, removing the transport medium for enthalpic cooling leaving porous $\mathrm{ZrO}_{2}$ layer, increasing the front face temperature due to a low thermal conductivity.

These results are faster than the time to equilibrium for the front face as seen in literature. The porous material will have the fastest rise in temperature for the front face as seen by Jia et al who reached equilibrium near $20 s$ while in a water cooled copper sample holer ${ }^{127}$. In another study of a porous UHTC in a water cooled sample holder Xie et al reported a time till equilibrium of over $100 s^{123}$. The conductivity will limit the back face temperature, therefore an over estimation of the $h$ value will not generate large inaccuracies in time to equilibrium. The time till equilibrium varied according to thermal conductivity, with the higher thermal conductivity corresponding to a lower time to equilibrium. The change in thermal conductivity from 96 to $10 \mathrm{~W} \mathrm{~m}^{-1} \mathrm{~K}^{-1}$ in dense and porous $\mathrm{TiB}_{2}$ doubled the time to equilibrium. 


\subsubsection{Equilibrium Temperature Profiles}

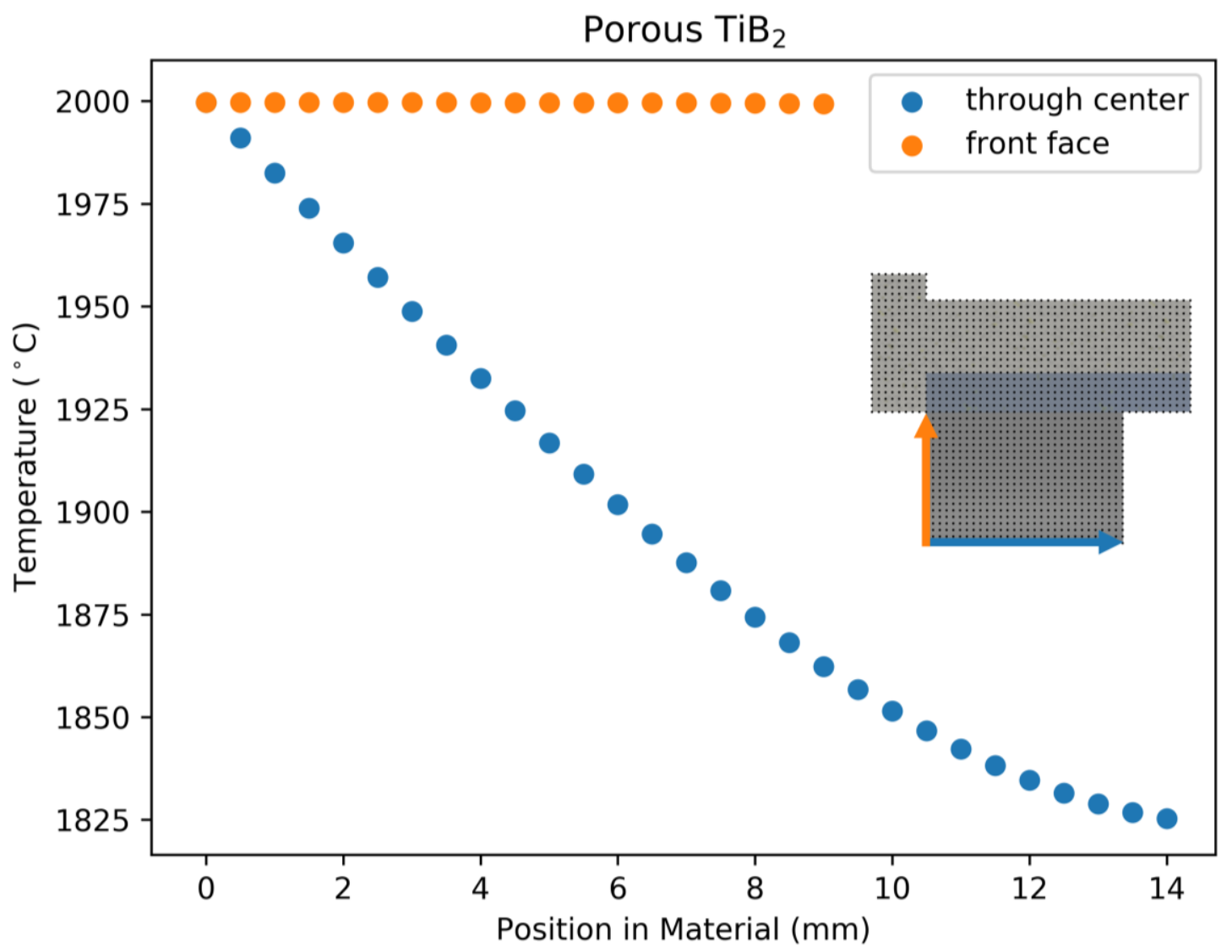

Figure 38: Temperature profile of porous $\mathrm{TiB}_{2}$ at equilibrium.

By surrounding our test material in the outer sleeve, we are attempting to insulate the material from heat loss and create a uniform temperature gradient within the sample. Figure 38 shows the front face temperature and the temperature through the center of the sample at equilibrium. The front face temperature is even across the sample radius. This uniformity is due to the even heat distribution provided by the torch and high convective heat transfer as defined in the model. In literature, the highest temperature read by a pyrometer at the center of the sample and no temperature distribution is reported. Paul et a $\left.\right|^{50,51}$ in two different studies reported a front face temperature distribution based on images from a thermal camera which 
was not as even as what is assumed in the model. When an oxyacetylene torch test is used as a screening test or for a qualitative oxidation study creating the harshest conditions is priority. There are several variables that can be altered in the torch set-up to allow for an even heating across the sample face. The torch head can be changed to produce a flame with larger area, the sample distance can be changed, and the gas ratios will additionally affect the heat distribution.

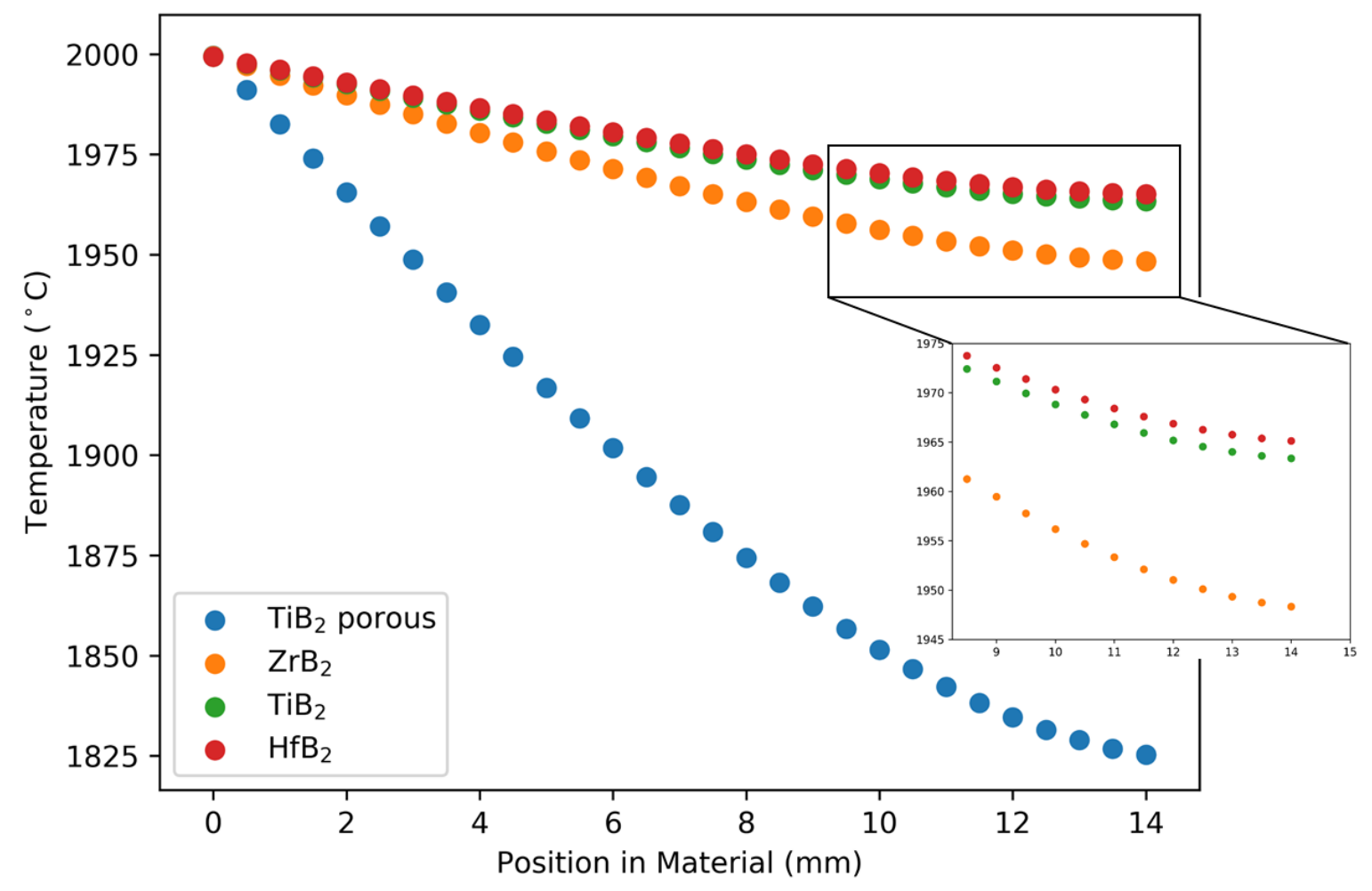

Figure 39:Temperature profiles through sample center at equilibrium of all materials modeled.

Figure 38 shows the temperature drop of several of the materials we modeled. The temperature drop within a material depends on the conductive heat transfer, a function of $k$, $C p$, and $\rho$. Conductivity has the greatest effect on the temperature drop. The material with the highest conductivity $\mathrm{HfB}_{2}$ at $104 \mathrm{~W} \mathrm{~m} \mathrm{~m}^{-1} \mathrm{~K}^{-1}$, has the lowest temperature drop of $25^{\circ} \mathrm{C}$, while 
porous $\mathrm{TiB}_{2}$ with the lowest conductivity at $10 \mathrm{~W} \mathrm{~m}^{-1} \mathrm{~K}^{-1}$, showed a much larger temperature drop of $184{ }^{\circ} \mathrm{C}$ across the radius. The non-linearity of the temperature profiles confirms that we cannot simply use temperature drop and an infinite plate assumption to back-calculate thermal conductivity.

Jia et al reported a temperature drop of over $1479^{\circ} \mathrm{C}$ from their $20 \%$ porous carbon/carbonZrC-SiC composite ${ }^{127}$. Jia et al used a water-cooled cooper sample holder with a $10 \mathrm{~mm}$ thick sample. The water-cooled copper holder alone can explain for the significant temperature drop. Miller-Oana et al reported the temperature drop for a $5 \mathrm{~mm}$ thick $\mathrm{ZrB}_{2}$ sample to be $147.5^{\circ} \mathrm{C}$ after $30 \mathrm{~s}$, while we estimate a temperature drop $51^{\circ} \mathrm{C}$ in a $14 \mathrm{~mm}$ thick sample. This discrepancy can come from several difference is our model set up and Miller-Oana et al's experimental setup. Our sample is insulated around the radius, and has a stagnant air convective transfer on the back face defined as $10 \mathrm{~W} \mathrm{~m}^{2} \mathrm{~K}$, not allowing for a tremendous amount of cooling. Miller-Oana et al had entire surface of the sample exposed to the flow surrounding flame, allowing the torch flow to affect the air flow behind the sample and therefore the convection. The samples were held by graphite studs, a medium conductive material at $27.5 \mathrm{~W} \mathrm{~m}^{-1} \mathrm{~K}^{-1} 121$. They also reported temperature drops across the radius of the sample front face of over $600^{\circ} \mathrm{C}$ this owes to the concentrated flame they were using. 


\section{$\underline{3.7 .3}$ Equilibrium Heat Maps}

a)

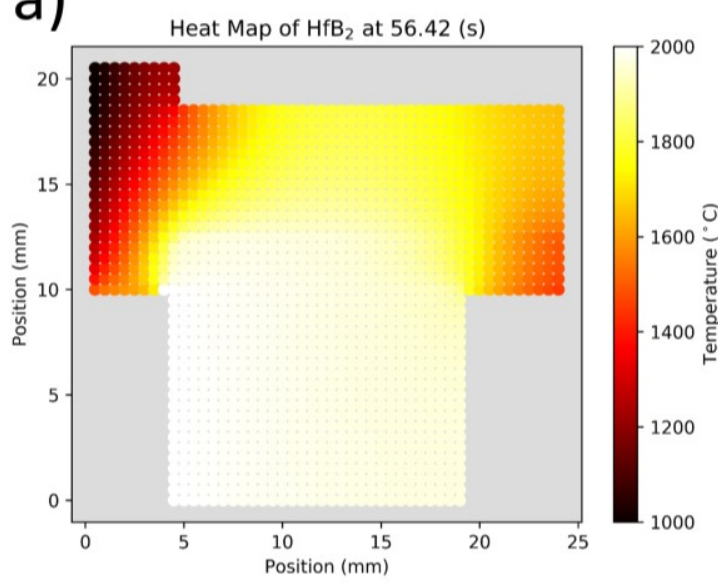

c)

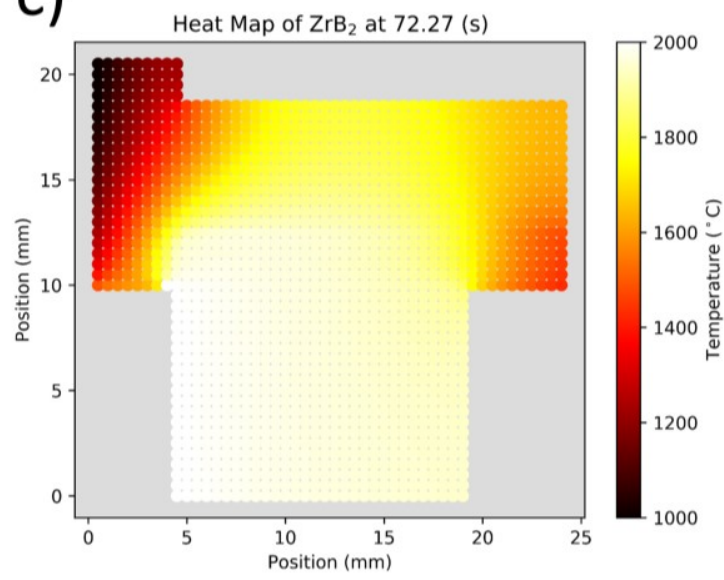

b)

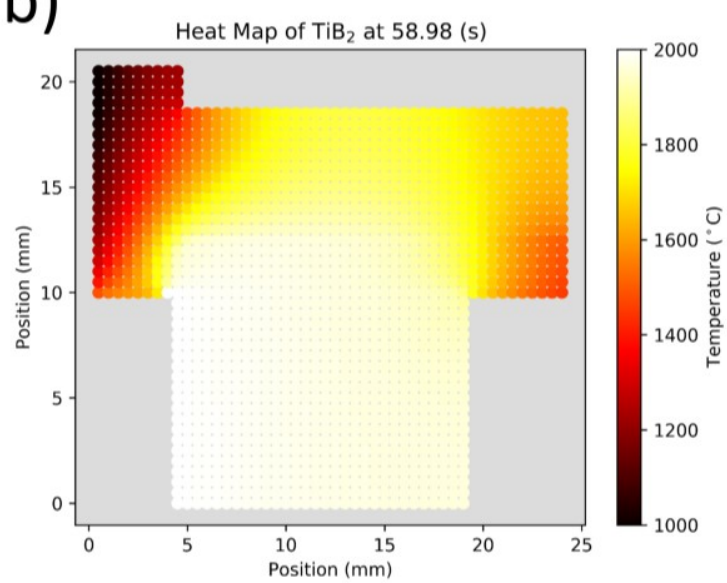

d)

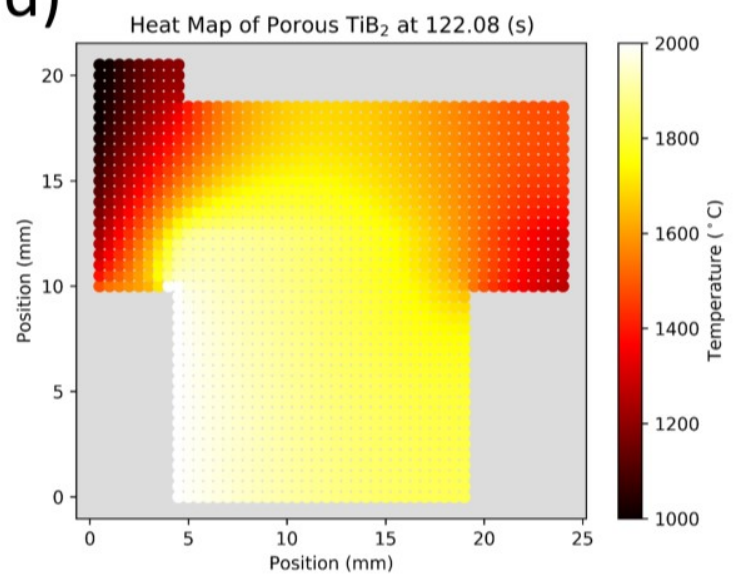

Figure 40: Equilibrium heat maps of a) dense $\mathrm{HfB}_{2}$, b) dense $\mathrm{TiB}_{2}, \mathrm{c}$ ) dense $\mathrm{ZrB}_{2}$ and d) $60 \mathrm{vol} \%$ poorous $\mathrm{TiB}_{2}$ at equlibrium in the last time step calculated by the model.

Heat maps of the entire material system sample holder and sample are shown in Figure 40 at equilibrium. This heat map is useful to validate that the temperature distribution is reasonable based on testing setup, conditions, and material properties. We can see that the radial heat flow is confined to the insulating felt surrounding the material providing a smooth consistent temperature gradient within the materials. The porous $\mathrm{TiB}_{2}$ has a noticeably different heat distribution, because of the lower conductivity. The heat distribution is not as consistent within the porous material, there is a low temperature area near the felt where the mold is removing 
heat from the sample. This emphasizes that sample size that is slightly shorter should be considered a to have an even temperature gradient.

\subsubsection{Oxide Layer Effect}

a)

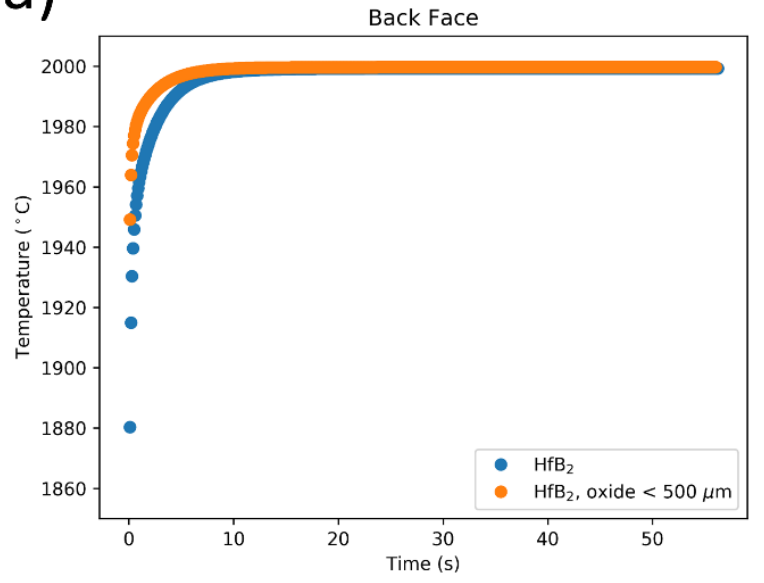

c)

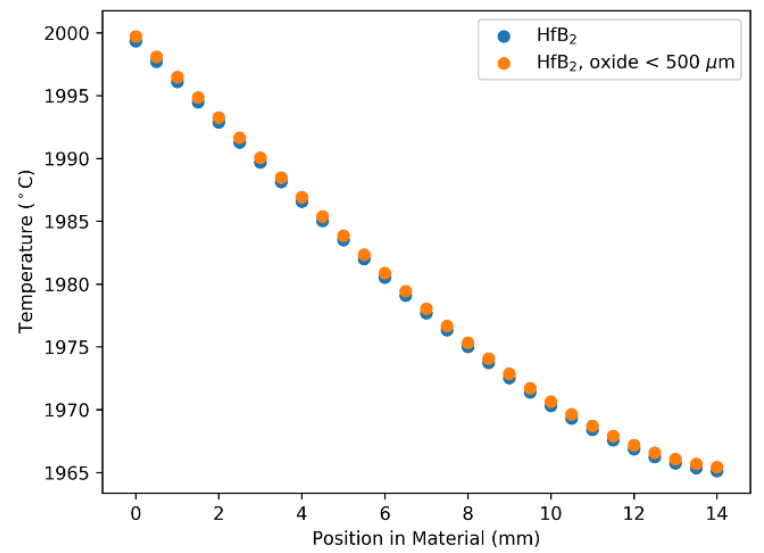

b)

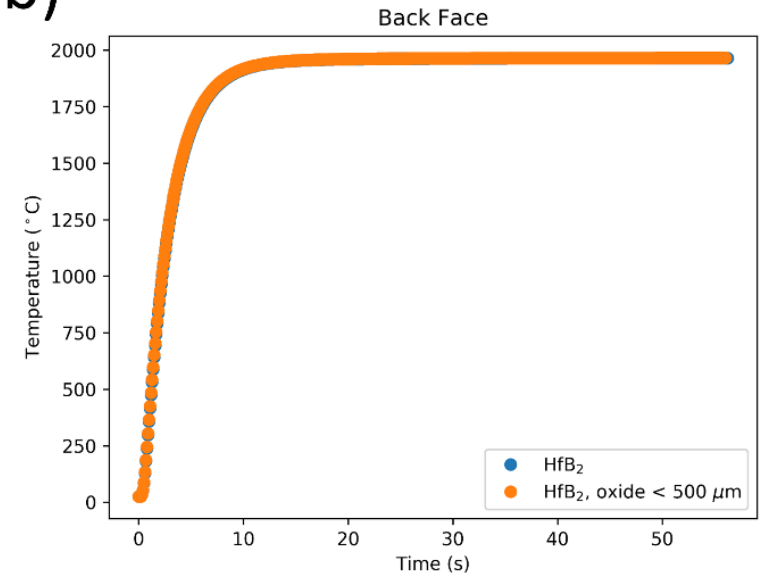

d)

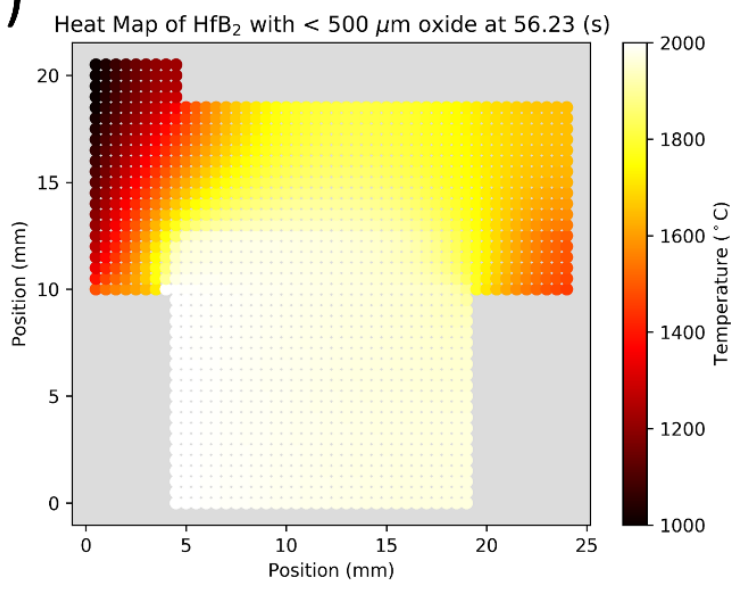

Figure 41: Oxide layer effect on a) heating of the front face, b) heating of the back face, c) equilibrium temperature through the center and d) equilibrium heat map.

The effects of oxide thickness below the node resolution of the model are plotted in Figure 41.

An oxide layer will increase in rate of temperature buildup on the front face because the oxide material typically has a lower combination of $C p$ and $\rho$ allowing the same torch $h$ value to raise the temperature of the material faster, shown in Figure $41 \mathrm{a}$. The bulk conductivity of the test 
material is still that of the non-oxidized material so the back face plots in Figure $41 \mathrm{~b}$ are similar. Figure $41 \mathrm{c}$ shows the temperature profile through the center of the test material and oxidized material. The test materials show similar curvature, with the oxidized material showing a slightly higher temperature through the whole material because of the lower $C p$ and $\rho$. The heat map in Figure $41 \mathrm{c}$ has a similar temperature distribution to that of Figure 40a. Defining the oxide contribution is a simplifying assumption that will have to be confirmed. In an oxyacetylene torch test there are several other factors that can contribute to the oxidation effect. $\mathrm{B}_{2} \mathrm{O}_{3}$ (Boria) liquid can form depending on the environment and both contribute to enthalpic cooling, convention and thermal conductivity of the oxide layer. As thickness of the oxide layer increases the conductivity effect will become stronger. The magnitude of these contributions will be tested with our torch rig and their effect validity of our solely convective transfer assumption. 


\section{$\underline{3.7 .5}$ Effective Thermal Conductivity}

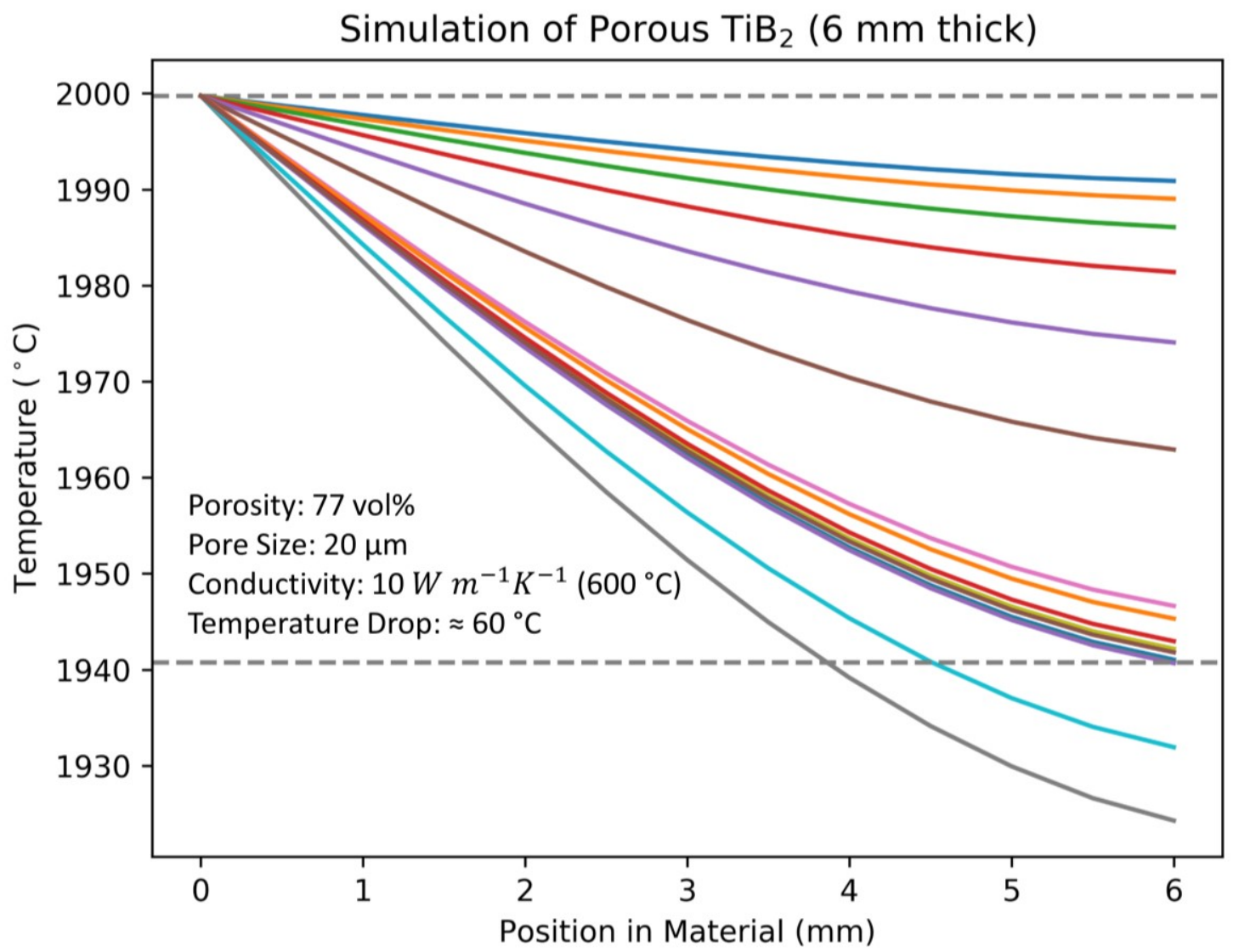

Figure 42: Equilibrium temperature drop values as model searches for conductivty.

The primary object of the model is to determine effective thermal conductivity. This will be done by inputting a temperature drop, heat capacity, and density to the model. The model will then iterate through thermal conductivity values till the corresponding temperature drop is achieved. Figure 42 shows numerous thermal conductivities being tried until the correct temperature drop is found for the case of a temperature drop from $77 \mathrm{vol} \%$ porous $\mathrm{TiB}_{2}$. The model will try new values until the set accuracy is reached. The temperature drop in Figure 42 is from the porous $\mathrm{TiB}_{2}$ model run. Using a previous runs temperature drop removes any error from the sample holder behaving different than described by the model. The models final 
prediction was $9.96 \mathrm{~W} \mathrm{~m}^{-1} \mathrm{~K}^{-1}, 1 \mathrm{~W} \mathrm{~m}^{-1} \mathrm{~K}^{-1}$ slightly lower than it should be, but that is limited by an accuracy limit set within the model. Higher precision could be used but will increase computation time, and may not be necessary when considering other sources of error when modeling from an experimental temperature drop. To test the accuracy and capabilities of the model for finding effective conductivity real tests must be conducted.

The porous materials modeled in this section are isotropic. The model does not yet have capability to input directional conductivity values based on pore structure variables, such as pore size, shape, organization, or distribution. Currently nodes can be given individual isotropic conductivity so multiple materials with composite structure larger than the node resolution can be studied. However, this type of structure will depend greatly on heat exchange at material boundaries and additional boundary rules will need to be added. In the future capability for anisotropic conductivity values will be added as well as interfacial resistance. We intend to measure the materials presented in Chapter 1 with the oxyacetylene torch rig, and compare to the measured conductivity results from Chapter 2. 


\subsubsection{Cooling Energy Balance}

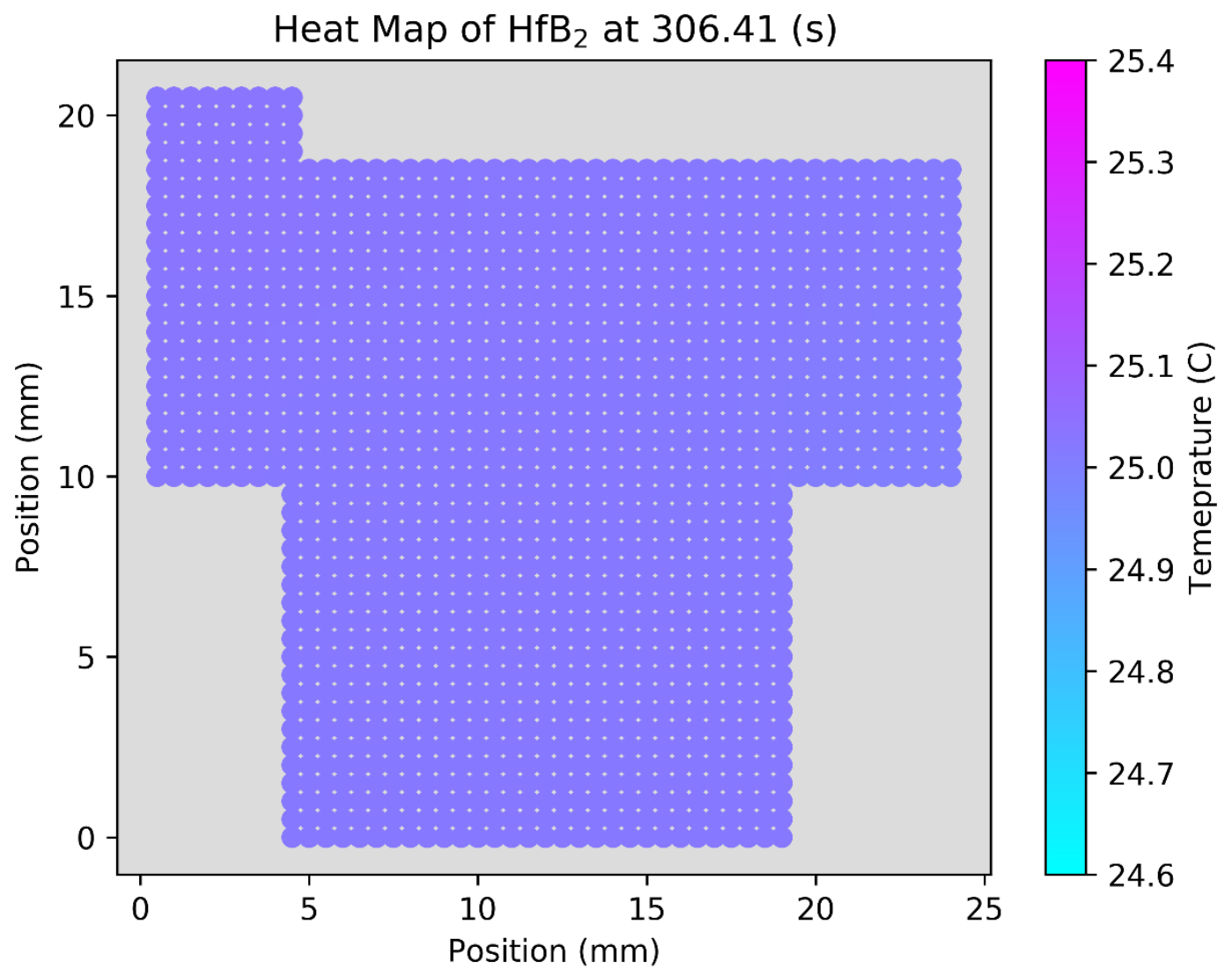

Figure 43: Heat map of a modeled cool time down of $\mathrm{HfB}_{2}$.

Figure 43 shows the heat distribution after the entire sample has cooled down. The cool down simulates a turning off of the torch and time to cool to the set ambient temperature of $25^{\circ} \mathrm{C}$. A cooling model allows us to check the energy balance to make sure we are passing heat correctly between nodes and there is no heat generation or excess heat loss in the model.

\subsection{Conclusion}

A model has been written to predict the equilibrium times and temperature of candidate materials for UHTC oxyacetylene torch testing, as well as output an effective thermal 
conductivity from tested materials. In order to remove some amounts of uncertainty, a sample holder designed of known materials incases the candidate UHTC. The model is based on a 2D finite difference energy balance across a set of defined nodes that cover a radial slice of the material and mold. Convective and conductive heat transport are the two mechanisms of heat transport used to compute the temperature change. The model currently follows connective flow assumptions that may need to be updated based on testing calibration. The model was able to produce temperature distributions across the front face, back face, heat maps and predict effective thermal conductivity based on a temperature drop. This work is important for understanding the dynamic behavior and conductivity of candidate materials like aligned porous $\mathrm{TiB}_{2}$. 


\section{Conclusion}

Highly anisotropic porous $\mathrm{TiB}_{2}$ structures have been produced as candidate materials for active and passive cooling. The outlined processing method relies on electrospun fibers as a sacrificial filler that can be altered to control the chemistry and morphology. $\mathrm{TiB}_{2}$ was made with pores of $5,6,8,10,12,15$ and $21 \mu m$, ranging from 56-72 vol \% porosity. The electrospun PS fibers facilitated the creation of aligned porous structures with tailored pore size and porosity.

Thermal conductivity and mechanical testing were conducted on the anisotropic porous material. Thermal conductivity measurements ranged from $12-31 \mathrm{~W} \mathrm{~m}^{-1} \mathrm{~K}^{-1}$ at RT with lower conductivity corresponding to higher porosity. The structure-property relationship was illuminated with a combined analytical thermal model, the interparticle porosity having the largest contribution. The mechanical behavior showed more progressive crushing behavior as porosity increased ranging in compressive strength from $0.4 \mathrm{MPa}$ at $70 \mathrm{vol} \%$ porosity to 30 $M P a$ at 59 vol \% porosity. The process demonstrated outperforms ice-templated $\mathrm{TiB}_{2}$ in the most significant property of thermal conductivity, and underperforms the ice-templated material with a still sufficient but slightly lower mechanical strength.

The data agreed with the Gibson Ashby honeycomb model for cellular materials. A finite difference 2D thermal model was developed to characterize UHTC materials under an oxyacetylene flame and estimate an effective thermal conductivity. This work serves to better define the processing-properties-structure relationship of aligned multiscale porous UHTCs, and improve the design space for hypersonic applications. 


\section{References}

1. Aylward M. Experiments in hyperspeed - USAASC. Army AL\&T. 2018.

2. Martinez OA, Sharma A, Sankar B V, et al. Thermal Force and Moment Determination of an Integrated Thermal Protection System. AIAA J. 2010;48:119-128.

https://doi.org/10.2514/1.40678

3. Savino R, De Stefano Fumo M, Paterna D, et al. Aerothermodynamic study of UHTC-based thermal protection systems. Aerosp Sci Technol. 2005;9:151-160.

https://doi.org/10.1016/j.ast.2004.12.003

4. Van Wie DM, Drewry DG, King DE, et al. The hypersonic environment: Required operating conditions and design challenges. J Mater Sci. 2004;39:5915-5924.

https://doi.org/10.1023/B:JMSC.0000041688.68135.8b

5. Opeka MM, Talmy I. ., Zaykoski J. Oxidation-based materials selection for $2000 \circ \mathrm{C}+$ hypersonic aerosurfaces : Theoretical. J Mater Sci. 2004;9:5887-5904.

https://doi.org/10.1023/B:JMSC.0000041686.21788.77

6. Glass D. Physical Challenges and Limitations Confronting the Use of UHTCs on Hypersonic Vehicles. 17th AIAA Int Sp Planes Hypersonic Syst Technol Conf. 2011:1-25.

https://doi.org/10.2514/6.2011-2304

7. Gogu C, Bapanapalli SK, Haftka RT, et al. Comparison of Materials for an Integrated Thermal Protection System for Spacecraft Reentry. J Spacecr Rockets. 2009;46:501-513. https://doi.org/10.2514/1.35669

8. Hald H. Operational limits for reusable space transportation systems due to physical boundaries of C/SiC materials. Aerosp Sci Technol. 2003;7:551-559. https://doi.org/10.1016/S1270- 
9638(03)00054-3

9. Tallon C, Slater S, Gillen A, et al. Ceramic Materials for Hypersonic Applications. Mater Aust. 2011:28-32.

10. Fahrenholtz WG, Wuchina EJ, Lee W, et al. Ultra High Temperature Ceramics: Materials for Extreme Environment Applications. 2008.

11. Fahrenholtz WG, Hilmas GE. Ultra-high temperature ceramics : Materials for extreme environments. Scr Mater. 2017;129:94-99. https://doi.org/10.1016/j.scriptamat.2016.10.018

12. Fahrenholtz WG, Hilmas GE, Talmy IG, et al. Refractory diborides of zirconium and hafnium. J Am Ceram Soc. 2007;90:1347-1364. https://doi.org/10.1111/j.1551-2916.2007.01583.x

13. Wuchina E, Opeka M, Causey S, et al. Designing for ultrahigh-temperature applications: The mechanical and thermal properties of HfB2, HfCx, HfNx and ??Hf(N). J Mater Sci. 2004;39:59395949. https://doi.org/10.1023/B:JMSC.0000041690.06117.34

14. Munro RG. Material properties of titanium diboride. J Res Natl Inst Stand Technol. 2000;105:709. https://doi.org/10.6028/jres.105.057

15. Brewer L, Sawyer D, Templeton D, et al. A Study of thee Refractory Borides. J Am Ceram Soc. 1951;34:173-179. https://doi.org/10.1088/1367-2630/11/11/113037

16. Tallon C, Franks GV. Exploring inexpensive processing routes to prepare dense TiB2 components. Adv Appl Ceram. 2016;115. https://doi.org/10.1080/17436753.2016.1172166

17. Galizia P, Zoli L, Sciti D. Impact of residual stress on thermal damage accumulation, and Young's modulus of fiber-reinforced ultra-high temperature ceramics. Mater Des. 2018;160:803-809. https://doi.org/10.1016/j.matdes.2018.10.019

18. Xin Y, Qizhong $\mathrm{H}$, Zhean S, et al. Ablative property and mechanism of C/C-ZrB2-ZrC-SiC 
composites reinforced by SiC networks under plasma flame. Corros Sci. 2016;107:9-20.

https://doi.org/10.1016/j.corsci.2016.02.015

19. Xu JZ, Gao BZ, Kang FY. A reconstruction of Maxwell model for effective thermal conductivity of composite materials. Appl Therm Eng. 2016;102:972-979.

https://doi.org/10.1016/j.applthermaleng.2016.03.155

20. Carson JK, Lovatt SJ, Tanner DJ, et al. An analysis of the influence of material structure on the effective thermal conductivity of theoretical porous materials using finite element simulations. Int J Refrig. 2003;26:873-880. https://doi.org/10.1016/S0140-7007(03)00094-X

21. Hu L, Wang CA, Huang Y, et al. Control of pore channel size during freeze casting of porous YSZ ceramics with unidirectionally aligned channels using different freezing temperatures. J Eur Ceram Soc. 2010;30:3389-3396. https://doi.org/10.1016/j.jeurceramsoc.2010.07.032

22. Tallon C. Multi-Scale Porous Ultra High Temperature Ceramics. 2015. https://doi.org/10.5817/CP2013

23. Monti R, Stefano Fumo M De, Savino R. Thermal Shielding of a Reentry Vehicle by Ultra-HighTempreature Ceramic Materials. J Thermophys Heat Transf. 2006;20:500-506. https://doi.org/10.2514/1.17947

24. Seuba J, Deville S, Guizard C, et al. Mechanical properties and failure behavior of unidirectional porous ceramics. Sci Rep. 2016;6:1-11. https://doi.org/10.1038/srep24326

25. Seuba J, Deville S, Guizard C, et al. The effect of wall thickness distribution on mechanical reliability and strength in unidirectional porous ceramics. Sci Technol Adv Mater. 2016;17:128135. https://doi.org/10.1080/14686996.2016.1140309

26. Trofimova EY, Kurdyukov DA. Porous ceramics mimicking nature - preparation and properties of microstructures with unidirectionally oriented pores. https://doi.org/10.1088/1468- 
$6996 / 12 / 6 / 064701$

27. Liu JX, Zhang GJ, Xu FF, et al. Densification, microstructure evolution and mechanical properties of WC doped HfB<inf>2</inf>-SiC ceramics. J Eur Ceram Soc. 2015;35:2707-2714. https://doi.org/10.1016/j.jeurceramsoc.2015.04.009

28. Ma H Bin, Man ZY, Liu JX, et al. Microstructures, solid solution formation and high-temperature mechanical properties of $\mathrm{ZrB}<\mathrm{inf}>2</ \mathrm{inf}>$ ceramics doped with $5 \mathrm{vol} . \% \mathrm{WC}$. Mater Des. 2015;81:133-140. https://doi.org/10.1016/j.matdes.2015.05.038

29. Zapata-Solvas E, Jayaseelan DD, Brown PM, et al. Effect of La2O3addition on long-term oxidation kinetics of ZrB2-SiC and HfB2-SiC ultra-high temperature ceramics. J Eur Ceram Soc. 2014;34:3535-3548. https://doi.org/10.1016/j.jeurceramsoc.2014.06.004

30. Hidrovo C, Goodson K. Active Microfluidic Cooling of Integrated Circuits. In: Bakir M, Meindl J, editors. Integrated Interconnect Technologies for 3D Nanoelectronic Syster. Norwood: Artech House Books; 2008:293-330.

31. Panigrahi PK. Transport Phenomena in Microfluidic Systems. Kanpur, India: John Wiley \& Sons; 2016.

32. ZHU Y, PENG W, XU R, et al. Review on active thermal protection and its heat transfer for airbreathing hypersonic vehicles. Chinese J Aeronaut. 2018;31:1929-1953.

https://doi.org/10.1016/j.cja.2018.06.011

33. Calister W, Rethwisch D. Materials Science and Engineering an Introduction. 9th ed. (Sayre D, editor.). Hoboken: John Wiley \& Sons; 2014.

34. Kasap SO. Principles of Electronic Materials and Devices. 2007.

35. Yan HY, Wei Q, Chang SM, et al. A first-principle calculation of structural, mechanical and 
electronic properties of titanium borides. Trans Nonferrous Met Soc China (English Ed.

2011;21:1627-1633. https://doi.org/10.1016/S1003-6326(11)60906-0

36. Pietrak K, Winiewski TS. A review of models for effective thermal conductivity of composite materials. Open Access J J Power Technol. 2015;95:14-24.

https://doi.org/10.1109/TPAMI.1986.4767851

37. Chauhan D, Singhvi N, Singh R. Dependence of effective thermal conductivity of composite materials on the size of filler particles. J Reinf Plast Compos. 2013;32:1323-1330.

https://doi.org/10.1177/0731684413490540

38. Gasch M, Johnson S, Marschall J. Thermal conductivity characterization of hafnium diboridebased ultra-high-temperature ceramics. J Am Ceram Soc. 2008;91:1423-1432.

https://doi.org/10.1111/j.1551-2916.2008.02364.x

39. Opeka MM, Talmy IG, Wuchina EJ, et al. Mechanical, Thermal, and Oxidation Properties of Refractory Hafnium and zirconium Compounds. J Eur Ceram Soc. 1999;19:2405-2414. https://doi.org/10.1016/S0955-2219(99)00129-6

40. Yuan C, Vandeperre LJ, Stearn RJ, et al. The effect of porosity in thermal shock. J Mater Sci. 2008;43:4099-4106. https://doi.org/10.1007/s10853-007-2238-x

41. Smith DS, Alzina A, Bourret J, et al. Thermal conductivity of porous materials. J Mater Res. 2013;28:2260-2272. https://doi.org/10.1557/jmr.2013.179

42. Wang R, Li W. Characterization models for thermal shock resistance and fracture strength of ultra-high temperature ceramics at high temperatures. Theor Appl Fract Mech. 2017;90:1-13. https://doi.org/10.1016/j.tafmec.2017.02.005

43. Studart AR, Gonzenbach UT, Tervoort E, et al. Processing routes to macroporous ceramics: A review. J Am Ceram Soc. 2006;89:1771-1789. https://doi.org/10.1111/j.1551-2916.2006.01044.x 
44. Paul A, Binner JGP, Vaidhyanathan B, et al. Oxyacetylene torch testing and microstructural characterization of tantalum carbide. J Microsc. 2013;250:122-129.

https://doi.org/10.1111/jmi.12028

45. Zhang X, Hu P, Han J, et al. Ablation behavior of ZrB2-SiC ultra high temperature ceramics under simulated atmospheric re-entry conditions. Compos Sci Technol. 2008;68:1718-1726. https://doi.org/10.1016/j.compscitech.2008.02.009

46. Liu Y, Fu Q, Wang B, et al. The ablation behavior and mechanical property of C/C-SiC-ZrB2 composites fabricated by reactive melt infiltration. Ceram Int. 2017;43:6138-6147. https://doi.org/10.1016/J.CERAMINT.2017.02.008

47. Tang S, Deng J, Wang S, et al. Ablation behaviors of ultra-high temperature ceramic composites. Mater Sci Eng A. 2007;465:1-7. https://doi.org/10.1016/J.MSEA.2007.02.040

48. Zhao $\mathrm{D}$, Zhang $\mathrm{C}$, $\mathrm{Hu} \mathrm{H}$, et al. Ablation behavior and mechanism of $3 \mathrm{D} \mathrm{C/ZrC} \mathrm{composite} \mathrm{in}$ oxyacetylene torch environment. Compos Sci Technol. 2011;71:1392-1396. https://doi.org/10.1016/J.COMPSCITECH.2011.05.005

49. Ang C, Williams T, Vowles D, et al. Influence of sol-gel derived ZrO2 and ZrC additions on microstructure and properties of ZrB2 composites. J Eur Ceram Soc. 2014;34:3139-3149. https://doi.org/10.1016/j.jeurceramsoc.2014.04.025

50. Paul A, Binner JGP, Vaidhyanathan B, et al. Heat flux mapping of oxyacetylene flames and their use to characterise Cf-HfB2composites. Adv Appl Ceram. 2016;115:158-165. https://doi.org/10.1080/17436753.2015.1104050

51. Paul A, Venugopal S, Binner JGP, et al. UHTC-carbon fibre composites: Preparation, oxyacetylene torch testing and characterisation. J Eur Ceram Soc. 2013;33:423-432. https://doi.org/10.1016/j.jeurceramsoc.2012.08.018 
52. Shi Y, Wan Y, Zhao D. Ordered mesoporous non-oxide materials. Chem Soc Rev. 2011;40:38543878. https://doi.org/10.1039/c0cs00186d

53. Studart R, Gonzenbach UT, Tervoort E, et al. Processing Routes to Macroporous Ceramics: A Review '. 2006;1789. https://doi.org/10.1111/j.1551-2916.2006.01044.x

54. Medri V, Mazzocchi M, Bellosi A. ZrB 2-based sponges and lightweight devices. Int J Appl Ceram Technol. 2011;8:815-823. https://doi.org/10.1111/j.1744-7402.2010.02512.x

55. Herzog A, Klingner R, Vogt $U$, et al. Wood-derived porous SiC ceramics by sol infiltration and carbothermal reduction. J Am Ceram Soc. 2004;87:784-793. https://doi.org/10.1111/j.15512916.2004.00784.x

56. Sakka Y, Tang F, Fudouzi $H$, et al. Fabrication of porous ceramics with controlled pore size by colloidal processing. Sci Technol Adv Mater. 2005;6:915-920. https://doi.org/10.1016/j.stam.2005.07.006

57. Hunter TN, Pugh RJ, Franks G V, et al. The role of particles in stabilising foams and emulsions. 2008;137:57-81. https://doi.org/10.1016/j.cis.2007.07.007

58. Chuanuwatanakul C, Tallon C, Dunstan DE, et al. Producing Large Complex-Shaped Ceramic Particle Stabilized Foams ‡. J Am Ceram Soc. 2013;1413:1407-1413. https://doi.org/10.1111/jace.12294

59. Malenfant PRL, Wan J, Taylor ST, et al. Self-assembly of an organic - inorganic block copolymer for nano-ordered ceramics. Nat Nanotechnol. 2007;2:43-46. https://doi.org/10.1038/nnano.2006.168

60. Isobe T, Kameshima Y, Nakajima A. Gas permeability and mechanical properties of porous alumina ceramics with unidirectionally aligned pores. 2007;27:53-59. https://doi.org/10.1016/j.jeurceramsoc.2006.02.030 
61. Hammond VH, Elzey DM. Elevated temperature mechanical properties of partially sintered alumina. Compos Sci Technol. 2004;64:1551-1563.

https://doi.org/10.1016/j.compscitech.2003.11.013

62. Teo WE, Ramakrishna S. A review on electrospinning design and nanofibre assemblies. Nanotechnology. 2006;17. https://doi.org/10.1088/0957-4484/17/14/R01

63. Zheng Y, Liu J, Liang J, et al. Graphitic carbon nitride materials: Controllable synthesis and applications in fuel cells and photocatalysis. Energy Environ Sci. 2012;5:6717-6731. https://doi.org/10.1039/c2ee03479d

64. Deville S, Meille S, Seuba J. A meta-analysis of the mechanical properties of ice-templated ceramics and metals. Sci Technol Adv Mater. 2015;16. https://doi.org/10.1088/1468$6996 / 16 / 4 / 043501$

65. Zhang G, Yang J, Ohji T. Fabrication of porous ceramics with unidirectionally aligned continuous pores. J Am Ceram Soc. 2001;97:1395-1397. https://doi.org/10.1111/j.11512916.2001.tb00849.x

66. Bae CJ, Erdonmez CK, Halloran JW, et al. Design of battery electrodes with dual-scale porosity to minimize tortuosity and maximize performance. Adv Mater. 2013;25:1254-1258. https://doi.org/10.1002/adma.201204055

67. Hsueh HY, Ho RM. Bicontinuous ceramics with high surface area from block copolymer templates. Langmuir. 2012;28:8518-8529. https://doi.org/10.1021/la3009706

68. Porter MM, Niksiar P, McKittrick J. Microstructural Control of Colloidal-Based Ceramics by Directional Solidification under Weak Magnetic Fields. J Am Ceram Soc. 2016;99:1917-1926. https://doi.org/10.1111/jace.14183

69. Halloran JW. Coextrusion Process. 2002;80:2578-2580. 
70. Crumm AT, Halloran JW. Negative Poisson's ratio structures produced from zirconia and nickel using co-extrusion. J Mater Sci. 2007;42:1336-1342. https://doi.org/10.1007/s10853-006-1209-y

71. Uyar T, Besenbacher F. Electrospinning of uniform polystyrene fibers: The effect of solvent conductivity. Polymer (Guildf). 2008;49:5336-5343.

https://doi.org/10.1016/j.polymer.2008.09.025

72. Fennessey S, Farris R. Fabrication of aligned and molecularly oriented electrospun polyacrylonitrile nanofibers and the mechanical behavior of their twisted yarns. Polymer (Guildf). 2004;45:4217-4225. https://doi.org/10.1016/j.polymer.2004.04.001

73. Anon. Polystyrene. Polymerdatabase.com. 2018.

74. Nishizaki H, Yoshida K. Effect of Molecular Weight on Various TGA Methods in Polystyrene Degradation. J Appl Polym Sci. 1981;26:3503-3504.

75. Kim SH, Kim YW, Park CB. Effect of inert filler addition on pore size and porosity of closed-cell silicon oxycarbide foams. J Mater Sci. 2004;39:3513-3515.

https://doi.org/10.1023/B:JMSC.0000026964.88284.ab

76. Isobe $\mathrm{T}$, Kameshima $\mathrm{Y}$, Nakajima A, et al. Preparation and properties of porous alumina ceramics with uni-directionally oriented pores by extrusion method using a plastic substance as a pore former. J Eur Ceram Soc Ceram Soc. 2007;27:61-66.

https://doi.org/10.1016/j.jeurceramsoc.2006.05.079

77. Jun SH, Chang MS, Kim BC, et al. Trypsin coatings on electrospun and alcohol-dispersed polymer nanofibers for a trypsin digestion column. Anal Chem. 2010;82:7828-7834.

https://doi.org/10.1021/ac101633e

78. Jun $\mathrm{SH}, \mathrm{Kim} \mathrm{K}, \mathrm{An} \mathrm{HJ}$, et al. Ethanol-dispersed polymer nanofibers as a highly selective cell isolation and release platform for CD4 + T lymphocytes. Adv Funct Mater. 2012;22:4448-4455. 
https://doi.org/10.1002/adfm.201200657

79. Yoon J, Yoon H-S, Shin Y, et al. Ethanol-dispersed and antibody-conjugated polymer nanofibers for the selective capture and 3-dimensional culture of EpCAM-positive cells. Nanomedicine Nanotechnology, Biol Med. 2017;13:1617-1625. https://doi.org/10.1016/j.nano.2017.02.015

80. Nair S, Kim J, Crawford B, et al. Improving biocatalytic activity of enzyme-loaded nanofibers by dispersing entangled nanofiber structure. Biomacromolecules. 2007;8:1266-1270. https://doi.org/10.1021/bm061004k

81. Ciesla U, Schüth F. Ordered mesoporous materials. Microporous Mesoporous Mater. 1999;27:131-149. https://doi.org/10.1016/S1387-1811(98)00249-2

82. Araki K, Halloran JW. Porous ceramic bodies with interconnected pore channels by a novel freeze casting technique. J Am Ceram Soc. 2005;88:1108-1114. https://doi.org/10.1111/j.15512916.2005.00176.x

83. Squire TH, Marschall J. Material property requirements for analysis and design of UHTC components in hypersonic applications. J Eur Ceram Soc. 2010;30:2239-2251. https://doi.org/10.1016/j.jeurceramsoc.2010.01.026

84. COLLISHAW PG, EVANS JRG. an Assessment of Expressions for the Apparent ThermalConductivity of Cellular Materials. J Mater Sci. 1994;29:2261-2273. https://doi.org/10.1007/BF00363413

85. Saxena SC, Chen SHP. Thermal conductivity of nitrogen in the temperature range 350-2500 K. Mol Phys. 1975;29:1507-1519. https://doi.org/10.1080/00268977500101321

86. Gibson L, Ashby MF. Cellular solids: Structure and properties. 1990. https://doi.org/10.2277/0521499119 
87. Francl J, Kingery W. Thermal Conductivity: IX, Experimental Investigation of Effect of Porosity on Thermal Conductivity. 1954;9:99-107. https://doi.org/10.1016/B978-0-08-092562-2.50019-9

88. Bhattacherjee D, King J, Whitehead KN. Thermal Conductivity of PU/PIR Foams as S a Function of Mean Temperature. J Cell Plast. 1991;27:240-251.

89. Li SY, Chu HS, Yan WM. Numerical study of phonon radiative transfer in porous nanostructures. Int J Heat Mass Transf. 2008;51:3924-3931. https://doi.org/10.1016/j.ijheatmasstransfer.2008.01.004

90. Hasselman DPH, Johnson LF. Effective Thermal Conductivity of Composites with Interfacial Thermal Barrier Resistance. J Compos Mater. 1987;21:508-515. https://doi.org/10.1177/002199838702100602

91. Ogushi T, Chiba H, Tane M, et al. Thermal Property of Lotus-Type Porous Copper and Application to Heat Sinks. Cell Porous Mater Therm Prop Simul Predict. 2008:239-265. https://doi.org/10.1002/9783527621408.ch8

92. Florez JPM, Mantelli MBH, Nuernberg GG V., et al. Powder Geometry Based Models for Sintered Media Porosity and Effective Thermal Conductivity. J Thermophys Heat Transf. 2014;28:507-517. https://doi.org/10.2514/1.T4206

93. Königshofer R, Fürnsinn S, Steinkellner P, et al. Solid-state properties of hot-pressed TiB2ceramics. Int J Refract Met Hard Mater. 2005;23:350-357. https://doi.org/10.1016/j.ijrmhm.2005.05.006

94. Rhee S. Porosity-Thermal Conductivity COrrelations for Ceramic Materials. Mater Sci Eng. 1975;20:89-93.

95. Tallon C, Chuanuwatanakul C, Dunstan DE, et al. Mechanical strength and damage tolerance of highly porous alumina ceramics produced from sintered particle stabilized foams. Ceram Int. 
2016;42:8478-8487. https://doi.org/10.1016/j.ceramint.2016.02.069

96. Rice RW. Evaluation and extension of physical property-porosity models based on minimum solid area. J Mater Sci. 1996;31:102-118. https://doi.org/10.1007/BF00355133

97. Boccaccini A, Ondracek G, Mombello E. Determination of stress concentration factors in porous materials. J Mater Sci .... 1996;14:534-536.

98. Gibson LJ. Modelling the mechanical behavior of cellular materials. Mater Sci Eng A. 1989;110:136. https://doi.org/10.1016/0921-5093(89)90154-8

99. Reynaud C, Thevenot F. Porosity dependence of mechanical properties of porous sintered SiC. Verification of the minimum solid area model. J Mater Sci Lett. 2000;19:871-874. https://doi.org/10.1023/A:1006741616088

100. Pabst W, Gregorová E. Minimum solid area models for the effective properties of porous materials - A refutation. Ceram - Silikaty. 2015;59:244-249.

101. Lam DCC, Lange FF, Evans AG. Mechanical Properties of Partially Dense Alumina Produced from Powder Compacts. J Am Ceram Soc. 1994;77:2113-2117. https://doi.org/10.1111/j.11512916.1994.tb07105.x

102. Boonyongmaneerat Y. Mechanical properties of partially sintered materials. Mater Sci Eng A. 2007;452-453:773-780. https://doi.org/10.1016/j.msea.2006.11.043

103. Nakajima H. Fabrication, properties and application of porous metals with directional pores. Prog Mater Sci. 2007;52:1091-1173. https://doi.org/10.1016/j.pmatsci.2006.09.001

104. Hyun SK, Nakajima H. Anisotropic compressive properties of porous copper produced by unidirectional solidification. Mater Sci Eng A. 2003;340:258-264. https://doi.org/10.1016/S09215093(02)00181-8 
105. E1461-13. Standard Test Method for Thermal Diffusivity by the Flash Method 1. ASTM Int.

2016;i:1-11. https://doi.org/10.1520/E1461-13.2.

106. Chen Z, Wang X, Giuliani F, et al. Microstructural characteristics and elastic modulus of porous solids. Acta Mater. 2015;89:268-277. https://doi.org/10.1016/j.actamat.2015.02.014

107. Meille S, Lombardi M, Chevalier J, et al. Mechanical properties of porous ceramics in compression: On the transition between elastic, brittle, and cellular behavior. J Eur Ceram Soc. 2012;32:3959-3967. https://doi.org/10.1016/j.jeurceramsoc.2012.05.006

108. Justin JF, Jankowiak A. Ultra High Temperature Ceramics: Densification, Properties and Thermal Stability. AerospaceLab J. 2011;3:AL3-08.

109. Han XY, Cheng HF, Xing X, et al. Microstructures and thermophysical properties of polymer derived SiC/C composites. Mech Eng Mater Pts 1-3. 2012;152-154:86-90. https://doi.org/10.4028/www.scientific.net/AMM.152-154.86

110. Gasch M, Johnson S. Thermal Conductivity Characterization of Hafnium Diboride-Based UltraHigh-Temperature Ceramics. 2008;1432:1423-1432. https://doi.org/10.1111/j.15512916.2008.02364.x

111. Zhang L, Pejaković DA, Marschall J, et al. Thermal and Electrical Transport Properties of Spark Plasma-Sintered HfB2 and ZrB2 Ceramics. J Am Ceram Soc. 2011;94:2562-2570. https://doi.org/10.1111/j.1551-2916.2011.04411.x

112. Lonergan JM, Fahrenholtz WG, Hilmas GE. Zirconium diboride with high thermal conductivity. J Am Ceram Soc. 2014;97:1689-1691. https://doi.org/10.1111/jace.12950

113. Yuan H, Li J, Shen Q, et al. Preparation and thermal conductivity characterization of ZrB2porous ceramics fabricated by spark plasma sintering. Int J Refract Met Hard Mater. 2013;36:225-231. https://doi.org/10.1016/j.ijrmhm.2012.09.003 
114. Kulpa A, Troczynski T. Oxidaion of TiB2 Powders below 900 C. J Am Ceram Soc. 1996;79.

115. Andrievskii RA, Shul'ga YM, Volkova LS, et al. Oxidation behavior of TiB.sub.2 micro- and nanoparticles. Inorg Mater VO - 52. 2016;52:686. https://doi.org/10.1134/S0020168516070013

116. Young-Hag K, Seung-Yong L, Hyoun-Ee K. Oxidation Behavior of Titanium Boride at Elevated Temperatures. J Ceram Soc Japan. 2001;104:785-787. https://doi.org/10.2109/jcersj.104.785

117. Lee DB, Lee YC, Kim DJ. The Oxidation of TiB2 Ceramics Containing Cr and Fe. Oxid Met. 2001;56:177-189. https://doi.org/10.1023/A:1010369526960

118. Ding Y, Xiao B. Anisotropic elasticity, sound velocity and thermal conductivity of TiO2polymorphs from first principles calculations. Comput Mater Sci. 2014;82:202-218.

https://doi.org/10.1016/j.commatsci.2013.09.061

119. Hirata $\mathrm{Y}$, Shimonosono $\mathrm{T}$, Sameshima $\mathrm{T}$, et al. Compressive mechanical properties of porous alumina powder compacts. Ceram Int. 2014;40:2315-2322. https://doi.org/10.1016/j.ceramint.2013.07.153

120. Chen H, Wang J, Chen H. Aerothermal Mode and Dynamic Analysis of Hypersonic Vehicle. 54th AIAA/ASME/ASCE/AHS/ASC Struct Struct Dyn Mater Conf. 2013. https://doi.org/10.2514/6.20131592

121. Miller-Oana $M$, Neff $P$, Valdez $M$, et al. Oxidation behavior of aerospace materials in high enthalpy flows using an oxyacetylene torch facility. J Am Ceram Soc. 2015;98:1300-1307. https://doi.org/10.1111/jace.13462

122. Li H jun, Yao X yuan, Zhang Y lei, et al. Effect of heat flux on ablation behaviour and mechanism of C/C-ZrB2-SiC composite under oxyacetylene torch flame. Corros Sci. 2013;74:265-270. https://doi.org/10.1016/j.corsci.2013.04.052 
123. Xie J, Li K, Li H, et al. Ablation behavior and mechanism of C/C-ZrC-SiC composites under an oxyacetylene torch at $3000^{\circ} \mathrm{C}$. Ceram Int. 2013;39:4171-4178.

https://doi.org/10.1016/j.ceramint.2012.10.273

124. Li H jun, Yao X yuan, Zhang Y lei, et al. Effect of heat flux on ablation behaviour and mechanism of C/C-ZrB2-SiC composite under oxyacetylene torch flame. Corros Sci. 2013;74:265-270. https://doi.org/10.1016/j.corsci.2013.04.052

125. Yan $\mathrm{M}, \mathrm{Li} \mathrm{H}, \mathrm{Fu}$ Q, et al. Ablative property of C/C-SiC-HfC composites prepared via precursor infiltration and pyrolysis under $3,000^{\circ} \mathrm{C}$ oxyacetylene torch. Acta Metall Sin (English Lett. 2014;27:981-987. https://doi.org/10.1007/s40195-014-0110-y

126. Han J, Hu P, Zhang X, et al. Oxidation-resistant ZrB2-SiC composites at $2200^{\circ} \mathrm{C}$. Compos Sci Technol. 2008;68:799-806. https://doi.org/10.1016/j.compscitech.2007.08.017

127. Jia Y, Yao X, Sun J, et al. Effect of ZrC particle size on the ablation resistance of C/C-ZrC-SiC composites. Mater Des. 2017;129:15-25. https://doi.org/10.1016/j.matdes.2017.05.019

128. Li KZ, Jing X, Qian-Gang F, et al. Effects of porous C/C density on the densification behavior and ablation property of C/C-ZrC-SiC composites. Carbon N Y. 2013;57:161-168. https://doi.org/10.1016/j.carbon.2013.01.059

129. Li Z, Li H, Zhang S, et al. Microstructures and ablation properties of C/C-SiC-ZrC composites prepared using C/C skeletons with various densities. Ceram Int. 2013;39:8173-8181. https://doi.org/10.1016/j.ceramint.2013.03.093

130. Yan C, Liu R, Cao Y, et al. Ablation behavior and mechanism of C/ZrC, C/ZrC-SiC and C/SiC composites fabricated by polymer infiltration and pyrolysis process. Corros Sci. 2014;86:131-141. https://doi.org/10.1016/j.corsci.2014.05.005

131. Corcoran S. Transport Phenomena in Materials Science \& Engineering. 1st ed. (Corcoran S, 
editor.). Blacksburg, VA: Virginia Tech; 2014. 


\section{Appendix A}

In order to perform a cross comparison of the ordered porous ceramic structures more quantitatively. An algorithm utilizing a 2D image of a porous structure was developed in this work to measure standardize the measure of inter-pore spacing as well as present a metric to quantify periodicity within these materials. The image is processed with Image using the particle analysis function to gather the centroids of the pores for use in this algorithm, in the form of a python program. Inter-pore spacing can be approximated by measuring the pore area and number against the total area imaged, but this measurement assumes a particular type of packing, and a perfectly periodic sample.

a)

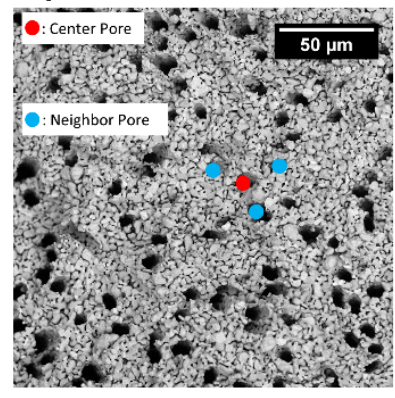

b)

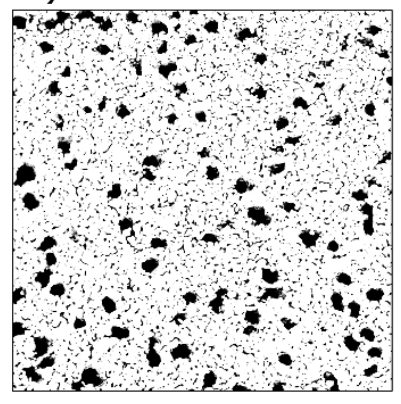

c)

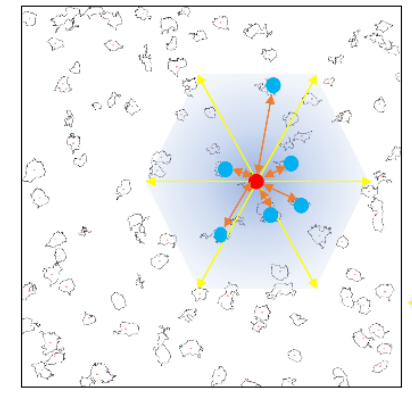

d)

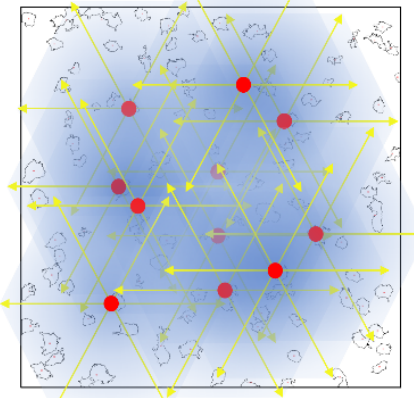

Figure 44: Visual 19 description of inter-pore spacing algorithm: (a) definition of center and neighbor pore overlaid on SEM image of $8 \mu \mathrm{m}$ pores, (b) SEM image converted to binary image, (b) outlines of pores used in measurement from ImageJ, using the "Analyze Particles..." function of the program with depiction of directional neighbor searching, and (d) increased overlay of pores searching for neighbors

In our algorithm, we estimate inter-pore spacing by measuring every pore against its neighbors.

To understand this process, we must first define neighbors: starting with a central pore, a neighbor is a pore is adjacent to the central pore in question, with direct line of sight to that central pore. Within our algorithm, neighbors can range from 3, lowest packed, to 6 hexagonal 
close packed. The area around a central pore is divided by the number of neighbors into a number of segments fanning out like a pie chart. All of the pores within each segment is collected, and the closest pore is stored as a neighbor. This is done for a central pore until all the neighbors are collected, and stored as distances. This measurement is done for every pore within the area of the image and averaged to give inter-pore spacing. The standard deviation of that inter-pore spacing is a measure of consistency in pore spacing or periodicity. This algorithm is applied across the 4 possible neighbor arrangements and the lowest standard deviation measurement is taken. 